- Supporting Information -

\title{
Nucleophilicity Parameters of Stabilized Iodonium Ylides for \\ Characterizing their Synthetic Potential
}

Saloua Chelli, ${ }^{\dagger}$ Konstantin Troshin, ${ }^{*}{ }^{\star}$ Peter Mayer, Sami Lakhdar, ${ }^{\dagger}$ Armin R. Ofial, and Herbert Mayr*

Department Chemie, Ludwig-Maximilians-Universität München, Butenandtstr. 5-13, 81377 München, Germany

* Corresponding Authors: kktroshin@gmail.com (K.T.), herbert.mayr@cup.uni-muenchen.de (H.M.);

${ }^{\dagger}$ Present address: ENSICAEN-Université de Caen Basse-Normandie, France

$\$$ Present address: University of California Berkeley, U. S. A. 


\section{Table of Contents}

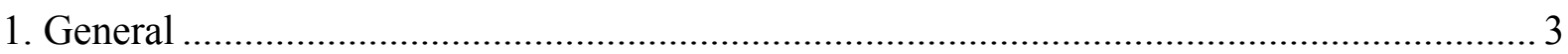

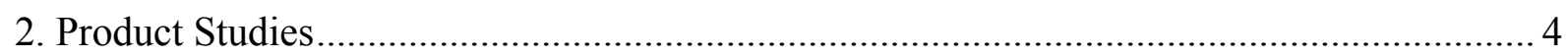

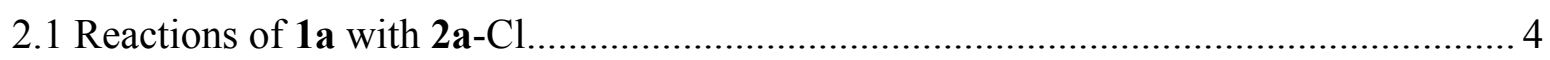

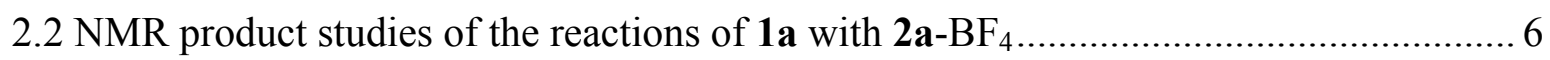

2.3 Reactions of iodonium ylides $\mathbf{1}$ with benzhydrylium ions $\mathbf{2}$ and aqueous workup......... 8

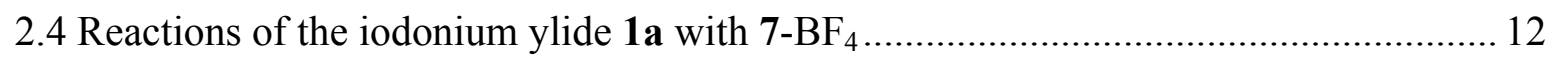

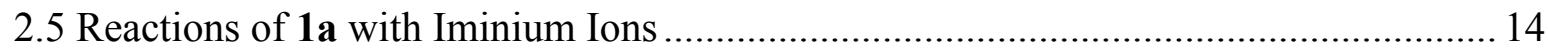

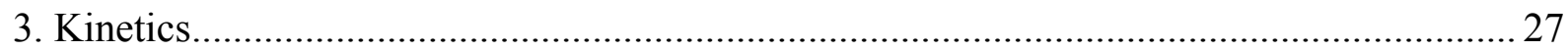

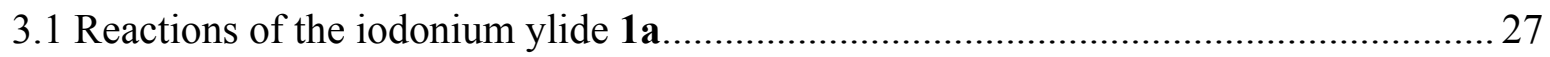

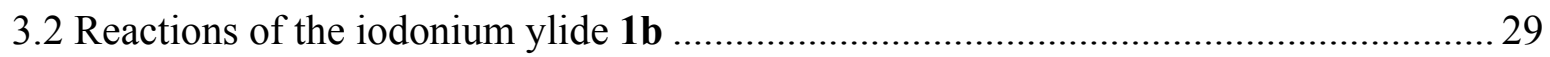

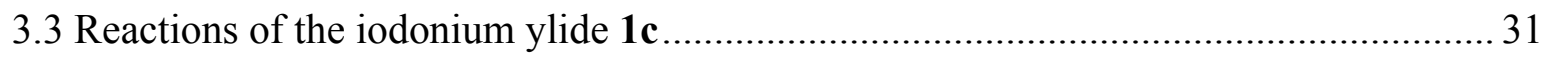

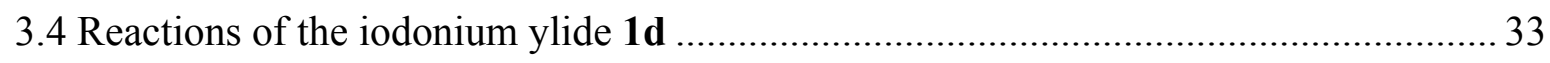

3.5 Reactions of the 6-methyl-2,4-dioxotetrahydro-2H-pyran-3-ide with electrophiles $\mathbf{2 i - k}$

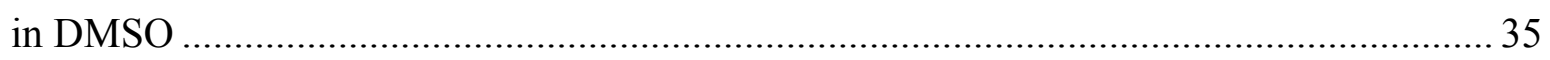

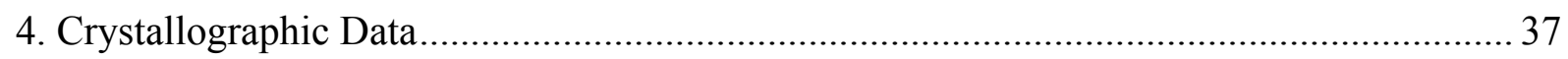

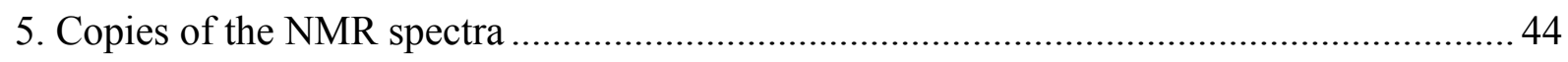

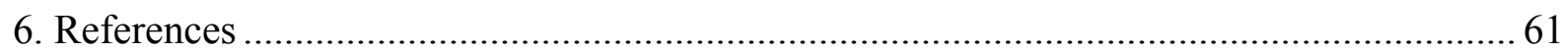




\section{General}

Chemicals. Iodonium ylides $\mathbf{1}(\mathbf{a}-\mathbf{c})$ were obtained using the procedure described by Charette $^{\mathrm{S} 1}$ and 1d was synthesized according to the method of Schank and Lick. ${ }^{\mathrm{S} 2}$ The benzhydrylium Ions $\mathbf{2 ( a - i ) ~ w e r e ~ p r e p a r e d ~ a s ~ d e s c r i b e d ~ b e f o r e . ~}{ }^{\mathrm{S} 3}$

Potassium 6-methyl-2,4-dioxotetrahydro-2H-pyran-3-ide was obtained by adding a solution of potassium tert-butoxide $(2.34 \mathrm{mmol}, 1 \mathrm{eq}, 263 \mathrm{mg})$ in $5 \mathrm{ml}$ of THF to a THF solution $(15 \mathrm{~mL})$ of 6-methyl-2.4-dioxotetrahydro-2H-pyran-3-ide (2.34 mmol, $1 \mathrm{eq}, 300 \mathrm{mg})$. The resulting colorless precipitate was collected by filtration, washed by anhydrous $n$-pentane and dried under vacuum (362 mg, 93\%).

Dichloromethane (p.a. grade) was subsequently treated with concentrated sulfuric acid, water, $10 \% \mathrm{NaHCO}_{3}$ solution, and again water. After pre-drying with anhydrous $\mathrm{CaCl}_{2}$, it was freshly distilled over $\mathrm{CaH}_{2}$. Acetonitrile (HPLC grade) was used as received.

Analytics. The ${ }^{1} \mathrm{H}$ and ${ }^{13} \mathrm{C}$ NMR chemical shifts $(\delta)$ are given in ppm and refer to $\mathrm{CDCl}_{3}\left(\delta_{\mathrm{H}}\right.$ $\left.=7.26, \delta_{\mathrm{C}}=77.16 \mathrm{ppm}\right)$, dichloromethane $\left(\delta_{\mathrm{H}}=5.32, \delta_{\mathrm{C}}=54.0 \mathrm{ppm}\right)$, or acetone $\left(\delta_{\mathrm{H}}=2.05\right.$, $\left.\delta_{\mathrm{C}}=29.84\right)$. Coupling constants are given in Hz. The following abbreviations are used to indicate the multiplicity: $\mathrm{s}$, singlet; $\mathrm{d}$, doublet; $\mathrm{t}$, triplet; $\mathrm{q}$, quartet; $\mathrm{m}$, multiplet. In the case of ${ }^{13} \mathrm{C}$ NMR spectra, these abbreviations refer to the multiplicity in proton-decoupled spectra, and hydrogen multiplicity (based on DEPT or HSQC experiments) is shown as $\mathrm{CH}_{3}, \mathrm{CH}_{2}$, $\mathrm{CH}$, or $\mathrm{C}$ to avoid ambiguity. NMR signal assignments are based on additional 2D NMR experiments (COSY, NOESY, HSQC, and HMBC).

Melting points were determined using a standard melting point unit with gradient of $0.5{ }^{\circ} \mathrm{C}$. HPLC studies were performed using DAICEL OD-H columns and heptane/isopropanol or and hexane/isopropanol mixtures as eluents.

Kinetics. The kinetics of the reactions of the iodonium ylides 1(a-d) with the benzhydrylium ions 2(a-f) in $\mathrm{CH}_{2} \mathrm{Cl}_{2}$ and of potassium 6-methyl-2,4-dioxotetrahydro-2H-pyran-3-ide with 
the benzhydrylium Ions 2(i-k) in DMSO were followed UV-vis spectroscopically by using conventional or stopped-flow techniques. The temperature $\left(20^{\circ} \mathrm{C}\right)$ was kept constant with a circulating bath thermostat.

\section{Product Studies}

\subsection{Reactions of 1 a with 2 a-Cl}

2-(Chloro(4-methoxyphenyl)methyl)-2-(4-methoxyphenyl)-5,5-dimethylcyclohexane-1,3dione (3) from the reaction of $2 \mathrm{a}-\mathrm{Cl}$ with $\mathbf{1 a}$ in the presence of $\mathrm{ZnCl}_{2} \cdot \mathrm{OEt}_{2}$ complex Zinc chloride (114 mg, $837 \mu \mathrm{mol})$, tetrabutylammonium chloride (190 mg, $685 \mu \mathrm{mol})$, diethyl ether $(0.10 \mathrm{~mL})$, and phenyliodonium-4,4-dimethylcyclohexane-2,6-dione 1a (569 mg, 1.66 mmol) were dissolved in dichloromethane $(6 \mathrm{~mL})$ and cooled to $-78{ }^{\circ} \mathrm{C}$. Then a solution of 4,4'-dimethoxybenzhydryl chloride $\mathbf{2 a}-\mathrm{Cl}(417 \mathrm{mg}, 1.59 \mathrm{mmol})$ in dichloromethane $(4 \mathrm{~mL})$ was added dropwise. The reaction solution was stirred at $-78 \mathrm{C}$ for $2 \mathrm{~h}$ and then quenched by addition of $2 \mathrm{M}$ aqueous ammonia. Diethyl ether was added to the organic phase followed by washing with water and brine, drying $\left(\mathrm{MgSO}_{4}\right)$, and evaporation of the solvents in a vacuum. The crude product was recrystallized from $\mathrm{Et}_{2} \mathrm{O} / n$-pentane (1/1): 3 (394 mg, $982 \mu$ mol, 62\%).

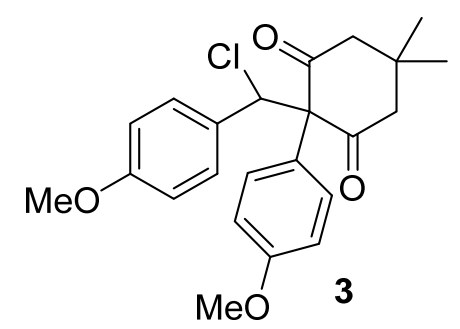

${ }^{1} \mathbf{H}$ NMR $\left(\mathrm{CDCl}_{3}, 300 \mathrm{MHz}\right): \delta 1.01\left(\mathrm{~s}, 6 \mathrm{H}, 2 \times \mathrm{CH}_{3}\right), 2.37-2.66\left(\mathrm{~m}, 4 \mathrm{H}, 2 \times \mathrm{CH}_{2}\right), 3.71(\mathrm{~s}$, $\left.3 \mathrm{H}, \mathrm{OCH}_{3}\right), 3.74\left(\mathrm{~s}, 3 \mathrm{H}, \mathrm{OCH}_{3}\right), 5.62(\mathrm{~s}, 1 \mathrm{H}, \mathrm{CH}), 6.54-6.73\left(\mathrm{~m}, 6 \mathrm{H}, \mathrm{H}_{\mathrm{Ar}}\right), 7.06-7.09(\mathrm{~m}, 2$ $\left.\mathrm{H}, \mathrm{H}_{\mathrm{Ar}}\right) .{ }^{13} \mathrm{C}$ NMR $\left(\mathrm{CDCl}_{3}, 75.5 \mathrm{MHz}\right): \delta 26.1\left(\mathrm{CH}_{3}\right), 30.6\left(\mathrm{CH}_{3}\right), 31.0(\mathrm{C}), 52.6\left(\mathrm{CH}_{2}\right), 53.8$ $\left(\mathrm{CH}_{2}\right), 55.3\left(\mathrm{OCH}_{3}\right), 55.4\left(\mathrm{OCH}_{3}\right), 62.3(\mathrm{CH}), 78.1(\mathrm{C}), 112.4(\mathrm{CH}), 114.8(\mathrm{CH}), 126.7(\mathrm{C})$, 
$129.2(\mathrm{CH}), 129.3(\mathrm{C}), 131.9(\mathrm{CH}), 158.8(\mathrm{C}), 159.9(\mathrm{C}), 204.6(\mathrm{C}=\mathrm{O}), 205.0(\mathrm{C}=\mathrm{O})$. HRMS (EI+): $m / z$ calcd for $\mathrm{C}_{23} \mathrm{H}_{25} \mathrm{ClO}_{4}\left[\mathrm{M}^{+}\right]: 400.1436$; Found: 400.1448 .

\section{Hydrolysis of 3}

The benzyl chloride 3 was dissolved in a $d_{6}$-acetone $/ \mathrm{D}_{2} \mathrm{O}$ mixture. The mixture was analyzed by NMR spectroscopy $12 \mathrm{~h}$ after mixing the reagents. The spectrum contained signals, which could be assigned to $p$-anisaldehyde (5a) by comparing with the previously reported data, ${ }^{\mathrm{S} 4}$ as well as resonances, which could be assigned to the rapidly equilibrating enol form of $\mathbf{6}$.

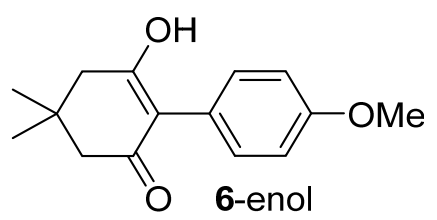

${ }^{1}$ H NMR (400 MHz, $d_{6}$-acetone/ $\left.\mathrm{D}_{2} \mathrm{O}=2 / 1\right): \delta 1.04(\mathrm{~s}, 6 \mathrm{H}), 2.36(\mathrm{~s}, 4 \mathrm{H}), 3.70$ (s, $\left.3 \mathrm{H}\right), 6.79$ $6.81(\mathrm{~m}, 2 \mathrm{H}), 6.99-7.01(\mathrm{~m}, 2 \mathrm{H}) .{ }^{13} \mathbf{C}$ NMR $\left(100.6 \mathrm{MHz}, d_{6}\right.$-acetone/ $\left.\mathrm{D}_{2} \mathrm{O}=2 / 1\right): \delta 28.0$ $\left(\mathrm{CH}_{3}\right), 31.9(\mathrm{C}), 47.1\left(\mathrm{CH}_{2}\right), 55.3\left(\mathrm{CH}_{3}\right), 113.6(\mathrm{CH}), 116.0(\mathrm{C}(\mathrm{C})=\mathrm{C}(\mathrm{C}) \mathrm{OH}), 126.2(\mathrm{C})$, $132.5(\mathrm{CH}), 158.5(\mathrm{C}), 186.0(\mathrm{C}=C(\mathrm{C})-\mathrm{OH})$.

\section{Reaction of 2a-Cl with 1a}

2a-Cl (156 mg, $0.594 \mathrm{mmol})$ and $\mathbf{1 a}(215 \mathrm{mg}, 0.628 \mathrm{mmol})$ were dissolved in dichloromethane and the resulting solution was stirred for $2 \mathrm{~h}$. The reaction mixture was freed from the solvent, and the crude product was analyzed by ${ }^{1} \mathrm{H}$ NMR (Figure S9, p S44) showing presence of $3(21 \%)$ and $4(36 \%)$. The crude mixture was then purified by column chromatography (silica, pentane/ethyl acetate $=4 / 1)$ leading to the isolation of $4(35 \mathrm{mg}$, $0.088 \mathrm{mmol}, 15 \%)$ as a colorless solid.

In order to obtain crystals suitable for X-ray diffraction analysis, 4 was recrystallized from $\mathrm{CH}_{2} \mathrm{Cl}_{2} /$ pentane. 


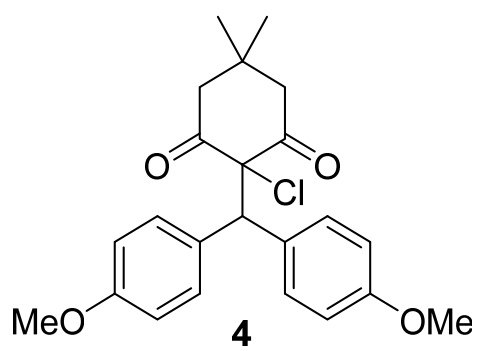

${ }^{1} \mathbf{H}$ NMR $\left(\mathrm{CDCl}_{3}, 300 \mathrm{MHz}\right): \delta 0.87(\mathrm{~s}, 3 \mathrm{H}), 1.15$ (s, $\left.3 \mathrm{H}\right), 2.66($ br d, $J=14.0 \mathrm{~Hz}, 2 \mathrm{H}), 2.98$ $(\mathrm{d}, J=14.0 \mathrm{~Hz}, 2 \mathrm{H}), 3.75(\mathrm{~s}, 6 \mathrm{H}), 5.10(\mathrm{~s}, 1 \mathrm{H}), 6.78-6.81(\mathrm{~m}, 4 \mathrm{H}), 7.25-7.29(\mathrm{~m}, 4 \mathrm{H}) .{ }^{13} \mathrm{C}$ NMR $\left(\mathrm{CDCl}_{3}, 75.5 \mathrm{MHz}\right): \delta 27.2\left(\mathrm{CH}_{3}\right), 30.0\left(\mathrm{CH}_{3}\right), 30.7(\mathrm{C}), 52.2\left(\mathrm{CH}_{2}\right), 55.3\left(\mathrm{OCH}_{3}\right), 56.1$ (CH), $89.9(\mathrm{C}), 113.8(\mathrm{CH}), 129.5(\mathrm{C}), 131.1(\mathrm{CH}), 159.1(\mathrm{C}), 198.2(\mathrm{C}=\mathrm{O})$.

\subsection{NMR product studies of the reactions of 1 a with $2 \mathrm{a}-\mathrm{BF}_{4}$}

\section{NMR investigation of the reaction between $2 \mathrm{a}-\mathrm{BF}_{4}$ and $1 \mathrm{a}$ (2 equiv.)}

Stock solutions of $\mathbf{2 a - B F} 4(80 \mu \mathrm{mol})$ and $\mathbf{1 a}\left(168 \mu \mathrm{mol}\right.$ in the resulting solution) in $\mathrm{CD}_{2} \mathrm{Cl}_{2}$ were mixed. In this case, full conversion of the cation to the species having broad ${ }^{1} \mathrm{H}$ NMR signals (200 MHz) was observed.

NMR investigation of the reaction between $2 a_{-}-B_{4}$ and 1 a (2 equiv.) with subsequent addition of $\mathrm{Bu}_{4} \mathrm{NCl}$

Stock solutions of $\mathbf{2} \mathbf{a}-\mathrm{BF}_{4}(63 \mu \mathrm{mol})$ and $\mathbf{1 a}(130 \mu \mathrm{mol})$ in $\mathrm{CD}_{2} \mathrm{Cl}_{2}$ were mixed. Immediately after the carbocation's color had faded (few seconds after the mixing), a $\mathrm{CD}_{2} \mathrm{Cl}_{2}$ solution of $\mathrm{Bu}_{4} \mathrm{NCl}(68 \mu \mathrm{mol})$ was added. The resulting solution was investigated by NMR spectroscopy. Sharp NMR signals were observed for all species, which were present in the solution. As depicted in Figures S10-S11 (pp S45-S46) several resonances were assigned to the structure I3a-Cl (based on the ${ }^{1} \mathrm{H}$ and ${ }^{13} \mathrm{C}$ NMR spectra and further COSY, HSQC, and HMBC experiments): 
${ }^{1} \mathbf{H}$ NMR $\left(400 \mathrm{MHz}, \mathrm{CD}_{2} \mathrm{Cl}_{2}\right): \delta 0.59(\mathrm{~s}, 3 \mathrm{H}, 12-\mathrm{H}$ or $13-\mathrm{H}), 0.97-0.99\left(3 \times 3 \mathrm{H}, 3 \times \mathrm{CH}_{3}, 12-\right.$ $\mathrm{H}$ or $13-\mathrm{H}, 19-\mathrm{H}, 20-\mathrm{H}$, overlapped with $\mathrm{Bu}_{4} \mathrm{~N}^{+}$signals but visible in $2 \mathrm{D}$ experiments), 2.16 (d, $\left.J=17.4 \mathrm{~Hz}, 1 \mathrm{H}, 14-\mathrm{H}^{\mathrm{a}}\right), 2.24-2.38(\mathrm{~m}, 2 \mathrm{H}, 10-\mathrm{H}), 2.39-2.45(\mathrm{~m}, 2 \mathrm{H}, 17-\mathrm{H}$ or $21-\mathrm{H})$, 2.75-2.83 (m, 2 H, 17-H or 21-H), $2.87\left(\mathrm{~d}, J=17.4 \mathrm{~Hz}, 1 \mathrm{H}, 14-\mathrm{H}^{\mathrm{b}}\right), 3.65$ (s, $\left.3 \mathrm{H}, 1-\mathrm{H}\right), 3.79$ (s, 3 H, 27-H), 6.10 (s, 1 H, 6-H), 6.39-6.42 (m, 2 H, 3-H), 6.51-6.56 (m, 2 H, 4-H), 6.716.76 (m, 2 H, 24-H), 6.86-6.92 (m, 2 H, 25-H), 7.36-7.41 (m, 2 H, 30-H), 7.51-7.56 (m, $1 \mathrm{H}$, 31-H), 8.01-8.03 (m, $2 \mathrm{H}, 29-\mathrm{H}) .{ }^{13} \mathbf{C}$ NMR (100.6 MHz, $\left.\mathrm{CD}_{2} \mathrm{Cl}_{2}\right): \delta 13.9\left(\mathrm{CH}_{3}, \mathrm{Bu}_{4} \mathrm{~N}^{+}\right), 20.3$ $\left(\mathrm{CH}_{2}, \mathrm{Bu}_{4} \mathrm{~N}^{+}\right), 24.5\left(\mathrm{CH}_{2}, \mathrm{Bu}_{4} \mathrm{~N}^{+}\right), 27.06\left(\mathrm{CH}_{3}, \mathrm{C}-19\right.$ or $\left.\mathrm{C}-20\right), 27.14\left(\mathrm{CH}_{3}, \mathrm{C}-12\right.$ or $\left.\mathrm{C}-13\right)$, $28.5\left(\mathrm{CH}_{3}, \mathbf{1 a}\right), 28.7\left(\mathrm{CH}_{3}, \mathrm{C}-12\right.$ or $\left.\mathrm{C}-13\right), 30.4\left(\mathrm{CH}_{3}, \mathrm{C}-19\right.$ or $\left.\mathrm{C}-20\right), 31.6(\mathrm{C}, \mathrm{C}-18), 32.3(\mathrm{C}$, 1a), $33.2(\mathrm{C}, \mathrm{C}-11), 41.5\left(\mathrm{CH}_{2}, \mathrm{C}-14\right), 50.0\left(\mathrm{CH}_{2}, \mathrm{C}-10\right), 51.2\left(\mathrm{CH}_{2}, 1 \mathrm{a}\right), 53.0\left(\mathrm{CH}_{2}, \mathrm{C}-17\right.$ or C-21), $53.8\left(\mathrm{CH}_{2}, \mathrm{C}-17\right.$ or $\left.\mathrm{C}-21\right), 55.6\left(\mathrm{CH}_{3}, \mathrm{C}-1\right), 56.0\left(\mathrm{CH}_{3}, \mathrm{C}-27\right), 59.5\left(\mathrm{CH}_{2}, \mathrm{Bu}_{4} \mathrm{~N}^{+}\right), 77.6$ (C, C-15), 83.4 (CH, C-6), 94.7 (C, PhI), 108.7 (C, C-8), 113.1 (CH, C-3), 115.4 (CH, C-25), 121.5 (C, C-28), 125.5 (C, C-23), 127.2 (C, C-5), 128.0 (CH, PhI), 130.6 (CH, C-24), 130.8 (CH, PhI), 130.9 (CH, C-4), 131.0 (CH, 1a), 131.1 (CH, C-31), 131.3 (CH, C-30), $131.6(\mathrm{CH}$, 1a), 133.3 (CH, 1a), 134.7 (CH, C-29), 137.9 (CH, PhI), 159.8 (C, C-2), 160.7 (C, C-26), 180.3 (C, C-7), 188.4 (C, 1a, C=O), 191.7 (C, C-9), 204.5 (C, C-16 or C-22), 205.6 (C, C-16 or C-22).

These assignments are supported by the following line of arguments: In the HMBC spectrum, the proton $6-\mathrm{H}$ has cross peaks with three of five low field signals lying in the region $\delta>175$ ppm (comparison with the NMR data for isolated 1a shows that one of them is the signal of the carbonyl groups of residual 1a). It is likely, therefore, that one of the dimedone moieties is attached to C-6 with its carbon (two cross peaks, since there are three bonds between 6-H and both carbonyl groups at C-16 and C-22), whereas the second dimedone moiety is attached with its oxygen (one cross peak, since there are three bonds between 6-H and enol carbon $\mathrm{C} 7$ but five bonds between 6-H and the carbon of the carbonyl group, C-9). Similarly to the keto form of unsubstituted dimedone $(\delta 203.7),{ }^{\mathrm{S} 5}$ the chemical shifts of C-16/C-22 $(\delta 204.5 / 205.6$ 
ppm) are much higher than those of C-7 $(\delta 180.3 \mathrm{ppm})$ and C-9 $(\delta 191.7 \mathrm{ppm})$, the latter pair of resonances being quite similar to the enol ether carbon ( $\delta 176.5 \mathrm{ppm})$ and the carbonyl group ( $\delta 198.9 \mathrm{ppm})$ of 3-methoxy-5,5-dimethyl-2-cyclohexen-1-one, respectively. ${ }^{\mathrm{S} 6}$ These chemical shifts may also be compared with those of the carbonyl groups in $\mathbf{3}(\delta 204.5,204.8$ ppm, keto form) and $\mathbf{6}$ ( $\delta 186.0 \mathrm{ppm}$, a superposition of a keto and enol signals as result of two rapidly equilibrating enol forms $)$. The chemical shifts of C-15 $(\delta 77.6 \mathrm{ppm})$ and $\mathrm{C}-17 / \mathrm{C}-$ $21(\delta 53.0 / 53.8 \mathrm{ppm})$ are also quite similar to those of the corresponding carbons in $\mathbf{3}(\delta 77.9$, 52.4/53.6 ppm), thus confirming the $\mathrm{C}$-attachment of the corresponding dimedone moiety. In contrast, the chemical shifts of C-8 ( $\delta 108.7 \mathrm{ppm}), \mathrm{C}-10(\delta 50.0 \mathrm{ppm})$, and C-14 ( $\delta 41.5 \mathrm{ppm})$ resemble a typical enol ether structure $(\delta 100.9,55.6,50.7$ ppm are reported for 3-methoxy5,5-dimethyl-2-cyclohexen-1-one in ref S6). The presence of oxygen atom attached to C-6 is also supported by the fact that its chemical shift $(\delta 83.4 \mathrm{ppm})$ is much higher than that of the corresponding carbon in $\mathbf{3}(\delta 62.1 \mathrm{ppm})$ carrying no oxygen.

When the reaction mixture generated as described above was stored for $48 \mathrm{~h}$ under inert atmosphere, disappearance of the $6-\mathrm{H}(\delta 6.10 \mathrm{ppm})$ resonance as well as appearance of a singlet at $\delta 5.62 \mathrm{ppm}$, a characteristic signal of $\mathbf{3}$, were observed.

\subsection{Reactions of iodonium ylides 1 with benzhydrylium ions 2 and aqueous workup}

5-Formyl-2,3-dihydrobenzofuran (5b). To a solution of $\mathbf{2 b}-\mathrm{Cl}(60.0 \mathrm{mg}, 0.209 \mathrm{mmol})$ in dichloromethane $(5 \mathrm{~mL})$, trimethylsilyl triflate $(222 \mathrm{mg}, 0.999 \mathrm{mmol})$ and $\mathbf{1 a}(216 \mathrm{mg}, 0.631$ mmol) were added successively. The resulting solution was stirred until the color of the benzhydrylium ion had disappeared. The reaction mixture was washed with water, dried over $\mathrm{MgSO}_{4}$, and freed from the solvent. The crude product was purified by column chromatography $\left(n\right.$-pentane $\left./ \mathrm{Et}_{2} \mathrm{O}=6 / 4\right): \mathbf{5 b}(20 \mathrm{mg}, 65 \%)$ as a light yellow oil. The ${ }^{1} \mathrm{H}$ NMR spectrum of $\mathbf{5 b}$ agreed with that described previously. ${ }^{\mathrm{S} 7}$ 


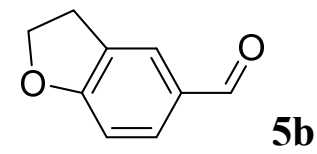

${ }^{1} \mathbf{H}$ NMR $\left(\mathrm{CDCl}_{3}, 400 \mathrm{MHz}\right): \delta 3.25-3.29(\mathrm{~m}, 2 \mathrm{H}), 4.69(\mathrm{t}, J=8.8 \mathrm{~Hz}, 2 \mathrm{H}), 6.88(\mathrm{~d}, J=8.2$ $\mathrm{Hz}, 1 \mathrm{H}), 7.65-7.68(\mathrm{~m}, 1 \mathrm{H}), 7.75(\mathrm{q}, J=1.5 \mathrm{~Hz}, 1 \mathrm{H}), 9.83(\mathrm{~s}, 1 \mathrm{H}) .{ }^{13} \mathbf{C} \mathbf{N M R}\left(\mathrm{CDCl}_{3}\right.$, 100.6 MHz): $\delta 28.9\left(\mathrm{CH}_{2}\right), 72.6\left(\mathrm{CH}_{2}\right), 109.8(\mathrm{CH}), 126.0(\mathrm{CH}), 128.6(\mathrm{C}), 130.6(\mathrm{C}), 133.2$, (CH), $165.8(\mathrm{C}), 190.7(\mathrm{CH})$.

\section{Reactions of the iodonium ylide 1a with benzhydrylium ions $2 \mathrm{c}-\mathrm{h}$}

General Procedure (GP1) for the syntheses of aldehydes 5: A solution of the iodonium ylide (3 equiv) in $\mathrm{CH}_{2} \mathrm{Cl}_{2}(0.1 \mathrm{~mol} / \mathrm{L})$ was added to a stirred dichloromethane solution of the benzhydrylium tetrafluoroborate $2-\mathrm{BF}_{4}(0.08 \mathrm{~mol} / \mathrm{L})$ at ambient temperature. The reaction solution was stirred until the blue color of the benzhydrylium ions had disappeared. The resulting mixture was quenched with aqueous $\mathrm{NaHCO}_{3}(10 \%)$. After separation of the liquid layers, the aqueous phase was extracted with $\mathrm{CH}_{2} \mathrm{Cl}_{2}$. The organic layers were combined, washed with brine, and dried $\left(\mathrm{MgSO}_{4}\right)$. After evaporation of volatiles under reduced pressure, the crude product was purified by column chromatography.

4-(Phenyl(2,2,2-trifluoroethyl)amino)benzaldehyde (5c). Following GP1, from 2c-BF (76.0 $\mathrm{mg}, 0.127 \mathrm{mmol})$ and $\mathbf{1 a}(130 \mathrm{mg}, 0.380 \mathrm{mmol}): \mathbf{5 c}(24.0 \mathrm{mg}, 0.086 \mathrm{mmol}, 68 \%)$ as a light yellow liquid.

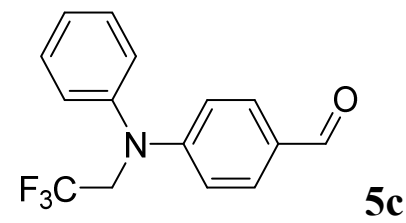

${ }^{1} \mathbf{H}$ NMR $\left(\mathrm{CDCl}_{3}, 300 \mathrm{MHz}\right): \delta 4.36\left(\mathrm{q}, J_{\mathrm{H}, \mathrm{F}}=8.5 \mathrm{~Hz}, 2 \mathrm{H}\right), 6.78-6.81(\mathrm{~m}, 2 \mathrm{H}), 7.24-7.27$ $(\mathrm{m}, 2 \mathrm{H}), 7.35-7.38(\mathrm{~m}, 1 \mathrm{H}), 7.45-7.51(\mathrm{~m}, 2 \mathrm{H}), 7.70-7.73(\mathrm{~m}, 2 \mathrm{H}), 9.81(\mathrm{~s}, 1 \mathrm{H}) .{ }^{13} \mathbf{C}$ $\operatorname{NMR}\left(\mathrm{CDCl}_{3}, 75.5 \mathrm{MHz}\right): \delta 53.9\left(\mathrm{q}, J_{\mathrm{C}, \mathrm{F}}=33.5 \mathrm{~Hz}, \mathrm{CH}_{2}\right), 114.5\left(\mathrm{q}, J_{\mathrm{C}, \mathrm{F}}=1.3 \mathrm{~Hz}, \mathrm{CH}\right), 124.9$ 
$\left(\mathrm{q}, J_{\mathrm{C}, \mathrm{F}}=283 \mathrm{~Hz}, \mathrm{C}\right), 127.5(\mathrm{CH}), 127.7\left(\mathrm{br} q, J_{\mathrm{C}, \mathrm{F}} \approx 1 \mathrm{~Hz}, \mathrm{CH}\right), 128.5(\mathrm{C}), 130.6(\mathrm{CH}), 131.7$

$(\mathrm{CH}), 145.3(\mathrm{C}), 153.2(\mathrm{C}), 190.5(\mathrm{CH})$. HRMS (ESI+): $\mathrm{m} / z$ calcd for $\mathrm{C}_{15} \mathrm{H}_{12} \mathrm{~F}_{3} \mathrm{NO}\left[\mathrm{M}^{+}\right]$: 279.0866; Found: 279.0858.

\section{4-(Methyl(2,2,2-trifluoroethyl)amino)benzaldehyde (5d).}

a) From ylide 1a: Following GP1, from $\mathbf{2 d - B F} 4(80 \mathrm{mg}, 0.168 \mathrm{mmol})$ and 1a (173 mg, 0.506 mmol): $\mathbf{5 d}(32 \mathrm{mg}, 88 \%$ ) as a light yellow liquid.

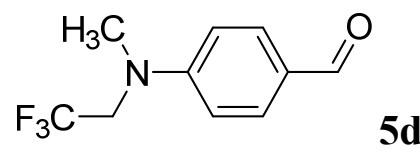

${ }^{1} \mathbf{H}$ NMR $\left(\mathrm{CDCl}_{3}, 400 \mathrm{MHz}\right) \delta 3.18(\mathrm{~s}, 3 \mathrm{H}), 3.99\left(\mathrm{q}, J_{\mathrm{H}, \mathrm{F}}=8.7 \mathrm{~Hz}, 2 \mathrm{H}\right), 6.84-6.87(\mathrm{~m}, 2 \mathrm{H})$, 7.78-7.80 (m, $2 \mathrm{H}), 9.81(\mathrm{~s}, 1 \mathrm{H}) .{ }^{13} \mathbf{C} \mathbf{N M R}\left(\mathrm{CDCl}_{3}, 100.6 \mathrm{MHz}\right): \delta 39.7\left(\mathrm{CH}_{3}\right), 53.6\left(\mathrm{q}, J_{\mathrm{C}, \mathrm{F}}\right.$ $\left.=33.6 \mathrm{~Hz}, \mathrm{CH}_{2}\right), 112.1(\mathrm{CH}), 125.2\left(\mathrm{q}, J_{\mathrm{C}, \mathrm{F}}=283 \mathrm{~Hz}, \mathrm{C}\right), 127.4(\mathrm{C}), 132.0(\mathrm{CH}), 153.1(\mathrm{C})$, 190.6 (CH). HMRS (ESI+): $m / z$ calcd for $\mathrm{C}_{10} \mathrm{H}_{10} \mathrm{~F}_{3} \mathrm{NO}\left[\mathrm{M}^{+}\right]$: 217.0709; Found: 217.0708.

5d from 2d and 1b. Following GP1, from 2d-BF 4 (80.0 mg, $0.168 \mathrm{mmol})$ and $\mathbf{1 b}(174 \mathrm{mg}$, $0.503 \mathrm{mmol}): \mathbf{5 d}(32.0 \mathrm{mg}, 88 \%)$ as a light yellow liqiud.

5d from 2d and 1c. Following GP1, from 2d-BF 4 (22.3 mg, $0.047 \mathrm{mmol})$ and 1c (50.5 mg, $0.141 \mathrm{mmol}): \mathbf{5 d}(6.6 \mathrm{mg}, 65 \%)$ as a light yellow liqiud.

5d from $2 d$ and 1d. Following GP1, from $2 d-B_{4}(20.0 \mathrm{mg}, 0.042 \mathrm{mmol})$ and $\mathbf{1 d}(41.4 \mathrm{mg}$, $0.503 \mathrm{mmol}): \mathbf{5 d}(4.8 \mathrm{mg}, 53 \%)$ as a light yellow liqiud.

4-(Diphenylamino)benzaldehyde (5e). Following GP1, from 2e-BF 4 (70.0 mg, 0.119 mmol) and 1a (124 mg, $0.362 \mathrm{mmol}): \mathbf{5 e}(22.0 \mathrm{mg}, 68 \%)$ as a light yellow solid; mp $128-130{ }^{\circ} \mathrm{C}$. The ${ }^{1} \mathrm{H}$ and ${ }^{13} \mathrm{C}$ NMR spectra of $\mathbf{5 e}$ matched those previously reported. ${ }^{\mathrm{S} 8}$

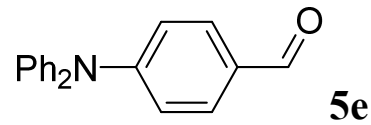


HRMS (ESI+): $m / z$ calcd for $\mathrm{C}_{19} \mathrm{H}_{16} \mathrm{NO}\left[\mathrm{M}+\mathrm{H}^{+}\right]$: 274.1226; Found: 274.1224.

4-Morpholinobenzaldehyde (5f). Following GP1, from $\mathbf{2 f - B F} 4(68.0 \mathrm{mg}, 0.160 \mathrm{mmol})$ and 1a (164 mg, $0.479 \mathrm{mmol})$ : 5f (19.5 mg, $64 \%$ ) as a light yellow oil. The ${ }^{1} \mathrm{H}$ and ${ }^{13} \mathrm{C} \mathrm{NMR}$ spectra of $\mathbf{5 f}$ matched those reported in the literature. ${ }^{\mathrm{S} 9}$

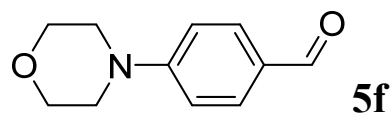

4-(Methylphenylamino)benzaldehyde (5g). Following GP1, from $\mathbf{2 g}-\mathrm{BF}_{4}$ (36 mg, 0.078 mmol) and 1a (80 mg, $0.234 \mathrm{mmol}): \mathbf{5 g}(11.4 \mathrm{mg}, 69 \%)$ as a light yellow liquid. The ${ }^{1} \mathrm{H}$ and ${ }^{13} \mathrm{C}$ NMR spectra of $\mathbf{5 g}$ matched those reported in the literature. ${ }^{\mathrm{S} 10}$

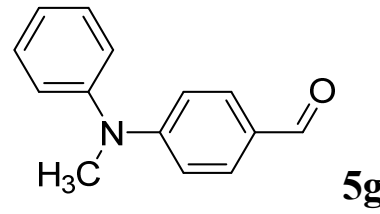

4-(Dimethylamino)benzaldehyde (5h). Following GP1, from $\mathbf{2 h}-\mathrm{BF}_{4}(60.0 \mathrm{mg}, 0.176 \mathrm{mmol})$ and 1a (181 mg, $0.529 \mathrm{mmol})$ : $\mathbf{5 h}(17.0 \mathrm{mg}, 65 \%)$ as a colorless solid; $\mathrm{mp} 72-73{ }^{\circ} \mathrm{C}$. The ${ }^{1} \mathrm{H}$ and ${ }^{13} \mathrm{C}$ NMR spectra of $\mathbf{5 h}$ matched those reported in the literature. ${ }^{\mathrm{S} 11}$

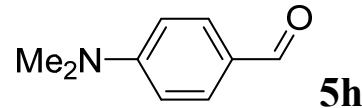




\subsection{Reactions of the iodonium ylide 1 a with $7-\mathrm{BF}_{4}$}

\section{2-(4-(Dimethylamino)phenyl)-2-(4a-(4-(dimethylamino)phenyl)-7,7-dimethyl-5-oxo-}

4a,5,6,7-tetrahydro-2H-chromen-2-yl)-5,5-dimethylcyclohexane-1,3-dione (8) and (E)-3(1-(4-(dimethylamino)phenyl)-4,4-dimethyl-2,6-dioxocyclohexyl)acrylaldehyde (9) from the reaction of the iodonium ylide $1 \mathrm{a}$ with $7-\mathrm{BF}_{4}$

7-BF $4(112 \mathrm{mg}, 0.306 \mathrm{mmol})$ and $\mathbf{1 a}(417 \mathrm{mg}, 1.22 \mathrm{mmol})$ were dissolved in $\mathrm{CH}_{2} \mathrm{Cl}_{2}$ (synthetic grade, $3 \mathrm{~mL}$ ) and the resulting mixture was stirred for $24 \mathrm{~h}$ at room temperature. The reaction solution was then quenched with saturated aq $\mathrm{NaHCO}_{3}(2 \mathrm{~mL})$. After extraction with $\mathrm{CH}_{2} \mathrm{Cl}_{2}$, the organic layers were combined, washed with brine, and dried $\left(\mathrm{MgSO}_{4}\right)$. The crude product was purified by column chromatography $\left(n\right.$-pentane/Et $\left.{ }_{2} \mathrm{O}=7 / 3\right): 8(86 \mathrm{mg}, 51$ $\%)$ and 9 (24 mg, $25 \%)$.

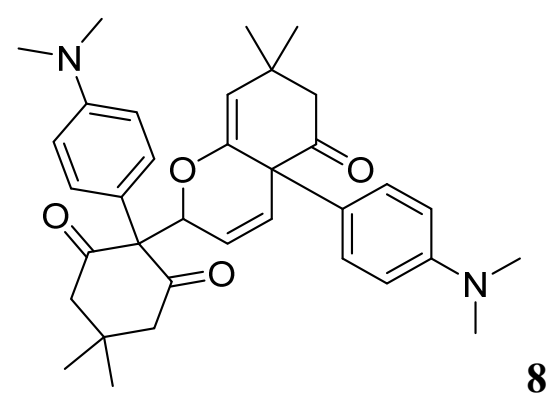

5,5-Dimethylcyclohexane-1,3-dione 8: light yellow crystals, mp $230{ }^{\circ} \mathrm{C}$, decomp. ${ }^{\mathbf{1}} \mathbf{H}$ NMR $\left(\mathrm{CDCl}_{3}, 400 \mathrm{MHz}\right): \delta 1.00(\mathrm{~s}, 3 \mathrm{H}), 1.03(\mathrm{~s}, 3 \mathrm{H}), 1.09(\mathrm{~s}, 3 \mathrm{H}), 1.11(\mathrm{~s}, 3 \mathrm{H}), 1.97(\mathrm{dd}, J=$ 12.6, 1.4 Hz, $1 \mathrm{H}), 2.39-2.48$ (m, $2 \mathrm{H}), 2.52-2.59$ (m, $2 \mathrm{H}), 2.66-2.71(\mathrm{~m}, 1 \mathrm{H}), 2.909$ (s, 6 H), 2.915 (s, $6 \mathrm{H}), 4.92-4.95(\mathrm{~m}, 2 \mathrm{H}), 5.59$ (dd, $J=10.0,2.1 \mathrm{~Hz}, 1 \mathrm{H}), 6.58-6.64(\mathrm{~m}, 4 \mathrm{H})$, 6.77-6.81 (m, $3 \mathrm{H}), 7.19-7.23(\mathrm{~m}, 2 \mathrm{H}) .{ }^{13} \mathbf{C ~ N M R}\left(\mathrm{CDCl}_{3}, 100.6 \mathrm{MHz}\right): \delta 26.3\left(\mathrm{CH}_{3}\right), 30.6$ (br, $\left.2 \times \mathrm{CH}_{3}\right), 31.0(\mathrm{C}), 31.6\left(\mathrm{CH}_{3}\right), 34.1(\mathrm{C}), 40.3\left(\mathrm{CH}_{3}\right), 40.6\left(\mathrm{CH}_{3}\right), 48.8\left(\mathrm{CH}_{2}\right), 52.2\left(\mathrm{CH}_{2}\right)$, $53.9(\mathrm{C}), 54.5\left(\mathrm{CH}_{2}\right), 74.4(\mathrm{CH}), 75.0(\mathrm{C}), 112.1(\mathrm{CH}), 112.6(\mathrm{CH}), 112.8(\mathrm{CH}), 119.4(\mathrm{C})$, $125.7(\mathrm{CH}), 126.2(\mathrm{CH}), 128.0(\mathrm{CH}), 128.2(\mathrm{C}), 128.5(\mathrm{CH}), 145.7(\mathrm{C}), 149.5(\mathrm{C}), 150.2(\mathrm{C})$, 205.3 (C), 205.9 (C), 206.2 (C). HRMS (ESI+): $m / z$ calcd for $\mathrm{C}_{35} \mathrm{H}_{43} \mathrm{~N}_{2} \mathrm{O}_{4}\left[\mathrm{M}+\mathrm{H}^{+}\right]: 555.3217$; Found: 555.3211. 


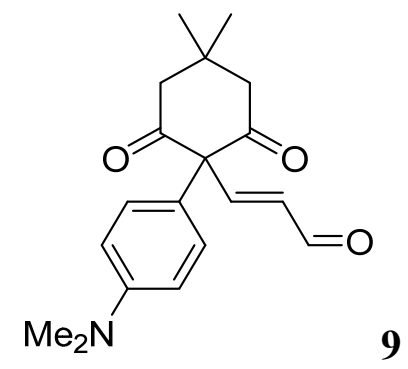

Acrylaldehyde 9: yellow crystals, mp $148-150^{\circ} \mathrm{C} .{ }^{1} \mathbf{H}$ NMR $\left(\mathrm{CDCl}_{3}, 400 \mathrm{MHz}\right): \delta 0.93$ (s, 3 H), 1.09 (s, 3 H), 2.41-2.46 (m, 2 H), 2.89-2.92 (m, 2 H), 2.97 (s, 6 H), 5.56 (dd, $J=15.7,7.9$ $\mathrm{Hz}, 1 \mathrm{H}), 6.66-6.70(\mathrm{~m}, 2 \mathrm{H}), 6.74-6.80(\mathrm{~m}, 2 \mathrm{H}), 7.16$ (d, $J=15.7 \mathrm{~Hz}, 1 \mathrm{H}), 9.63$ (d, $J=7.9$ $\mathrm{Hz}, 1 \mathrm{H}) .{ }^{13} \mathrm{C}$ NMR $\left(\mathrm{CDCl}_{3}, 100.6 \mathrm{MHz}\right): \delta 26.4\left(\mathrm{CH}_{3}\right), 30.4\left(\mathrm{CH}_{3}\right), 31.6(\mathrm{C}), 40.3\left(\mathrm{CH}_{3}\right)$, $52.5\left(\mathrm{CH}_{2}\right), 113.2(\mathrm{CH}), 122.1(\mathrm{C}), 128.6(\mathrm{CH}), 136.9(\mathrm{CH}), 150.4(\mathrm{C}), 155.4(\mathrm{CH}), 193.5$ (C), 205.3 (C). HRMS (ESI+) calcd for $\mathrm{C}_{19} \mathrm{H}_{24} \mathrm{NO}_{3}\left[\mathrm{M}+\mathrm{H}^{+}\right]$: 314.1751; Found: 314.1750.

Reaction of $\mathbf{1 a}$ with 7 in the presence of water. $7-\mathrm{BF}_{4}(15.6 \mathrm{mg}, 42.6 \mu \mathrm{mol})$ and $\mathbf{1 a}(39.2 \mathrm{mg}$, $115 \mu \mathrm{mol})$ were dissolved in $\mathrm{CD}_{2} \mathrm{Cl}_{2}(0.55 \mathrm{~mL})$ followed by addition of $9 \mathrm{mg}$ (1 drop, 0.5 mmol) of $\mathrm{D}_{2} \mathrm{O}$. The ${ }^{1} \mathrm{H}$ NMR spectrum acquired 5 min after the mixing of the reactants showed formation of a complex mixture containing 9 and some unidentified products. After 70 min of reaction time, some of the unidentified products were converted into 9 resulting in a significant increase of its concentration. The ${ }^{1} \mathrm{H}$ NMR spectrum remained unchanged after another $10 \mathrm{~h}$ of reaction time.

Reaction of 1 a with 7 in the presence of molecular sieves. In a glove box with dry argon atmosphere, 7-BF $4(20.0 \mathrm{mg}, 54.6 \mu \mathrm{mol})$ and $\mathbf{1 a}(56 \mathrm{mg}, 164 \mu \mathrm{mol})$ were dissolved in $\mathrm{CD}_{2} \mathrm{Cl}_{2}$ followed by addition of molecular sieves (ca $50 \mathrm{mg}$ ). The ${ }^{1} \mathrm{H}$ NMR spectrum acquired after 2 $\mathrm{h}$ of reaction time showed formation of $\mathbf{8}$ and some unidentified products whereas no traces of 9 were detected. The molecular sieves were then removed. $\mathrm{D}_{2} \mathrm{O}(9 \mathrm{mg}, 1 \mathrm{drop}, 0.5 \mathrm{mmol})$ was added to the resulting mixture and the resulting sample was analyzed by ${ }^{1} \mathrm{H}$ NMR $10 \mathrm{~h}$ after the addition. Only traces of $\mathbf{9}$ were detected in the spectrum, whereas the characteristic signal of 8 at 4.9 ppm remained unchanged. 


\subsection{Reactions of 1a with Iminium Ions}

Preparation of 6,6-dimethyl-4,8-dioxo-2-phenylspiro[2.5]octane-1-carbaldehyde (rac-12)

a) From (E)-1-(3-phenylallylidene)pyrrolidin-1-ium triflate and iodonium ylide 1a:<smiles>[O-]/C=C/C=[N+]1CC[C+](/C=C/c2ccccc2)C1</smiles>

$1 \mathrm{a}$

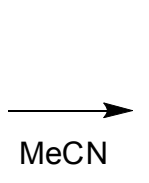

$\mathrm{Ph}^{\prime \prime}$
$(1 R, 2 S)$<smiles>CC1(C)CC(=O)C2(C(=O)C1)[C@H](c1ccccc1)[C@H]2C=O</smiles>

rac-12

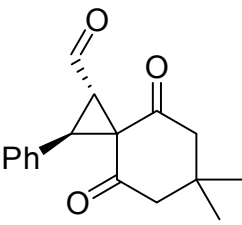

$(1 S, 2 R)$

(E)-1-(3-Phenylallylidene)pyrrolidin-1-ium triflate $(31.1 \mathrm{mg}, 92.7 \mu \mathrm{mol})$ and $1 \mathbf{a}(31.7 \mathrm{mg}$, 92.6 $\mu \mathrm{mol})$ were dissolved in $\mathrm{MeCN}(10 \mathrm{~mL})$, and the resulting solution was stirred for $12 \mathrm{~h}$ at ambient temperature. The crude mixture was then analyzed by HPLC.

HPLC (OD-H column, eluent: 80/20 hexane/iPrOH, flow rate: $0.8 \mathrm{~mL} / \mathrm{min})$.

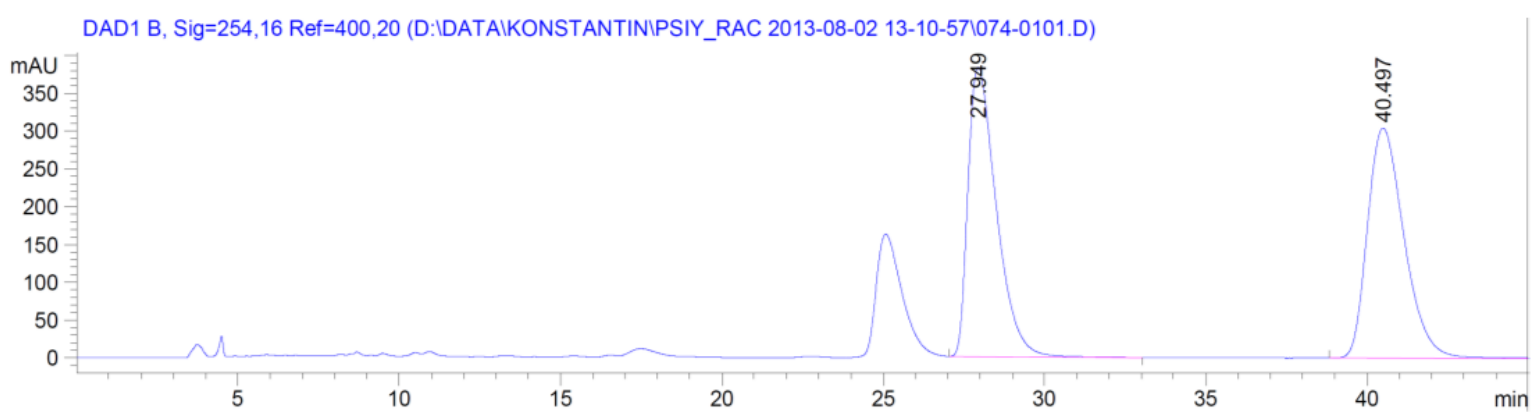

\begin{tabular}{|c|c|c|c|c|c|c|}
\hline $\begin{array}{c}\text { Peak } \\
\quad \#\end{array}$ & $\begin{array}{c}\text { RetTime } \\
\text { [min] }\end{array}$ & Type & $\begin{array}{l}\text { Width } \\
\text { [min] }\end{array}$ & $\begin{array}{c}\text { Area } \\
{\left[\mathrm{mAU}{ }^{\star} \mathrm{s}\right]}\end{array}$ & $\begin{array}{l}\text { Height } \\
{[\mathrm{mAU}]}\end{array}$ & $\begin{array}{c}\text { Area } \\
\quad \frac{\circ}{0}\end{array}$ \\
\hline . & & & $-------\mid$ & --------- & $----------\mid$ & $--------\mid$ \\
\hline 1 & 27.949 & $\mathrm{BB}$ & 0.9375 & $2.33452 \mathrm{e} 4$ & 382.80356 & 49.3989 \\
\hline 2 & 40.497 & $\mathrm{BBA}$ & 1.2196 & $2.39133 e 4$ & 304.04477 & 50.6011 \\
\hline
\end{tabular}


b) From cinnamaldehyde (14) and iodonium ylide 1a with morpholinium tetrafluoroborate ( 0.2 equiv) as the catalyst:<smiles>O=C/C=C/c1ccccc1</smiles><smiles>CC1(C)CC(=O)C([PH2+])C(=O)C1</smiles>

14

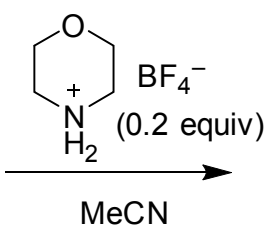

1a<smiles>CC1(C)CC(=O)C2(C(=O)C1)[C@H](c1ccccc1)[C@H]2C=O</smiles>

$(1 R, 2 S)$

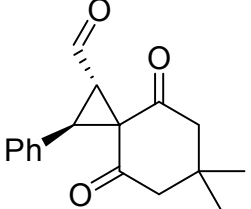

$(1 S, 2 R)$

Morpholinium tetrafluoroborate $(32.5 \mathrm{mg}, 57.7 \mu \mathrm{mol}), \mathbf{1 a}(98.7 \mathrm{mg}, 288 \mu \mathrm{mol})$, and 14 (41.9 mg, $317 \mu \mathrm{mol}$ ) were dissolved in $\mathrm{MeCN}$. The resulting mixture was stirred for $12 \mathrm{~h}$ at ambient temperature. Then an aliquot was taken from the crude mixture and analyzed by HPLC.

HPLC (OD-H column, eluent: 80/20 heptane/iPrOH, flow rate: $1.0 \mathrm{~mL} / \mathrm{min})$.

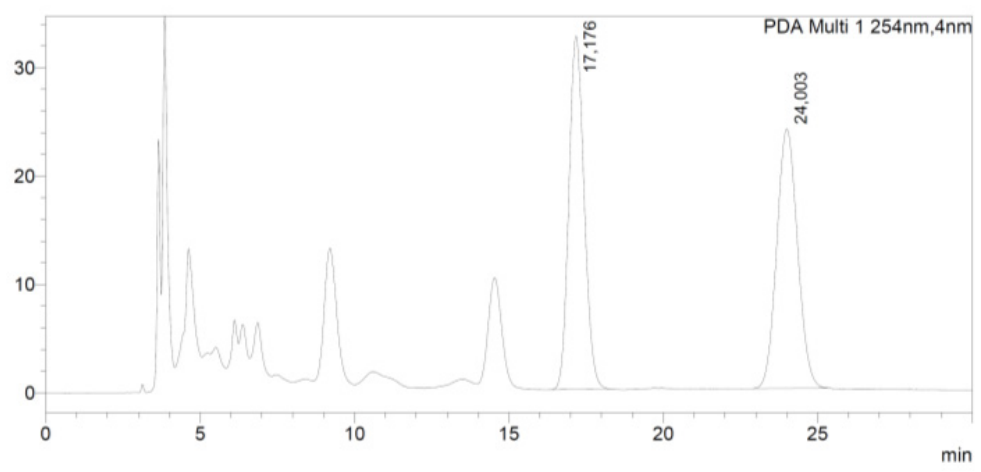

\begin{tabular}{|c|c|c|c|c|}
\hline \multirow{2}{*}{\multicolumn{2}{|c|}{$\begin{array}{l}\text { PDA Ch1 254nm } \\
\text { Peak\# Ret. Time }\end{array}$}} & & & \\
\hline & & Area & Height & Conc. \\
\hline 1 & 17,176 & 1123298 & 32608 & 49,961 \\
\hline 2 & 24,003 & 1125067 & 23948 & 50,039 \\
\hline Tota & & 2248365 & 56556 & \\
\hline
\end{tabular}




\section{Reaction of the iodonium ylide 1 a with $10 a-P_{6}$ (Table 4 , entry 1 )}

$(S)-10 a-P_{6}(239 \mathrm{mg}, 0.500 \mathrm{mmol})$ and $\mathbf{1 a}(171 \mathrm{mg}, 0.500 \mathrm{mmol})$ were dissolved in MeCN (5 $\mathrm{mL}$ ). The resulting solution was stirred for $24 \mathrm{~h}$ at ambient temperature. Then water was added $(10 \mathrm{~mL})$, and the phases were separated. The aqueous phase was extracted with $\mathrm{CH}_{2} \mathrm{Cl}_{2}$ $(15 \mathrm{~mL})$. The organic layers were combined, washed with brine, and dried over $\mathrm{MgSO}_{4}$. After evaporation of the solvent under vacuum, the crude product was purified by column chromatography (n-pentane/Et ${ }_{2} \mathrm{O}: 7 / 3$ and 6/4): 12 (98.0 mg, $0.363 \mathrm{mmol}, 72 \%$, e.r. $=59 / 41$ from HPLC) as colorless powder (mp $\left.124-126^{\circ} \mathrm{C}\right)$.

${ }^{1} \mathbf{H}$ NMR $\left(\mathrm{CDCl}_{3}, 400 \mathrm{MHz}\right): \delta 1.03(\mathrm{~s}, 3 \mathrm{H}), 1.15(\mathrm{~s}, 3 \mathrm{H}), 2.17-2.22(\mathrm{~m}, 1 \mathrm{H}), 2.39(\mathrm{dd}, J=$ 16.3, $2.0 \mathrm{~Hz}, 1 \mathrm{H}), 2.61-2.74(\mathrm{~m}, 2 \mathrm{H}), 3.68(\mathrm{dd}, J=8.7,4.6 \mathrm{~Hz}, 1 \mathrm{H}), 3.84(\mathrm{~d}, J=8.7 \mathrm{~Hz}, 1$ H), 7.20-7.22 (m, $2 \mathrm{H}), 7.30-7.34(\mathrm{~m}, 3 \mathrm{H}), 9.81(\mathrm{~d}, J=4.6 \mathrm{~Hz}, 1 \mathrm{H}) .{ }^{13} \mathbf{C} \mathbf{N M R}\left(\mathrm{CDCl}_{3}, 101\right.$ MHz) $\delta 27.7\left(\mathrm{CH}_{3}\right), 29.5\left(\mathrm{CH}_{3}\right), 30.7(\mathrm{C}), 42.1(\mathrm{CH}), 48.4(\mathrm{CH}), 53.85\left(\mathrm{CH}_{2}\right), 53.88\left(\mathrm{CH}_{2}\right)$, $57.2(\mathrm{C}), 128.6(\mathrm{CH}), 128.8(\mathrm{CH}), 129.4(\mathrm{CH}), 131.1(\mathrm{C}), 196.9(\mathrm{CH}), 198.7(\mathrm{C}), 204.9(\mathrm{C})$. HRMS (ESI+): $m / z$ calcd for $\mathrm{C}_{17} \mathrm{H}_{19} \mathrm{O}_{3}\left[\mathrm{M}+\mathrm{H}^{+}\right]$: 271.1329; Found: 271.1329.

For a single crystal x-ray structure of 6,6-dimethyl-4,8-dioxo-2-phenylspiro[2.5]octane-1carbaldehyde (12) see ref S12.

HPLC (OD-H column, eluent: 80/20 heptane/iPrOH, flow rate: $1.0 \mathrm{~mL} / \mathrm{min}$ ).

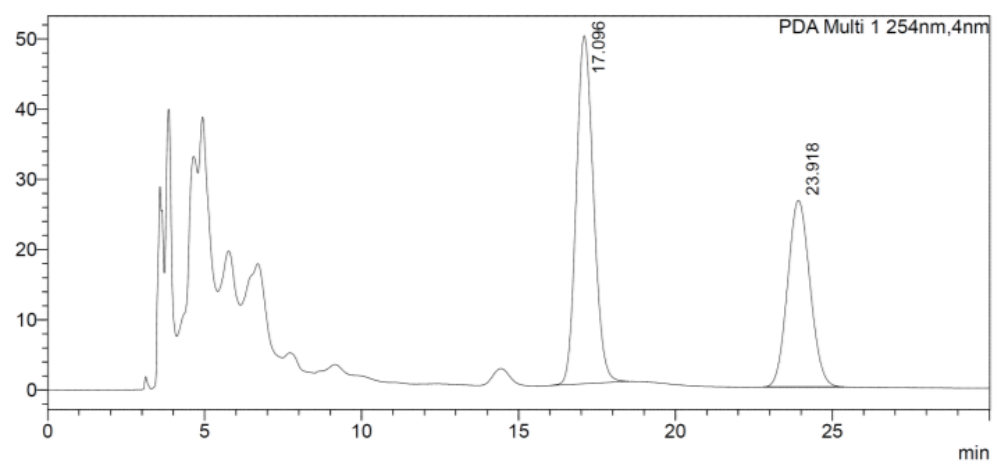

\begin{tabular}{|r|r|r|r|r|}
\hline Peak\# & Ret. Time & Area & Height & \multicolumn{1}{|c|}{ Conc. } \\
\hline 1 & 17.096 & 1892830 & 49540 & 58.915 \\
\hline 2 & 23.918 & 1320005 & 26581 & 41.085 \\
\hline Total & & 3212835 & 76121 & \\
\hline
\end{tabular}


General procedure for reactions of $1 \mathrm{a}$ with cinnamaldehyde (14) in the presence of 11aTFA. Aldehyde $14(0.17 \mathrm{mmol})$ and 11a-TFA $(0.034 \mathrm{mmol})$ were dissolved in the corresponding solvent and the resulting mixture was brought to the specified temperature followed by the addition of $\mathbf{1 a}(0.15 \mathrm{mmol})$ and stirring for a specified time. The resulting mixture was worked up as described above for $\mathbf{1 0 a}-\mathrm{PF}_{6}$ or analyzed directly by HPLC. All yields given in Table 4 of the main manuscript refer to the mass of the isolated product.

Table 4, entry 2:

Reaction in $\mathrm{MeCN}$ at $20^{\circ} \mathrm{C}$ for $12 \mathrm{~h}$

HPLC (OD-H column, eluent: $80 / 20$ hexane/iPrOH, flow rate: $0.8 \mathrm{~mL} / \mathrm{min}$ )

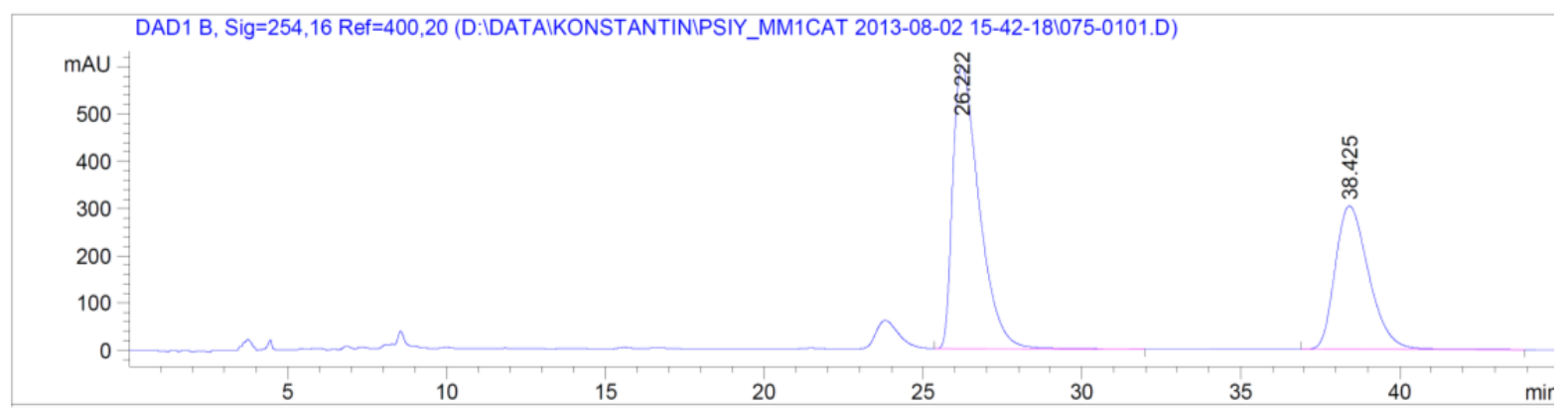

Signal 1: DAD1 B, Sig=254,16 Ref=400,20

\begin{tabular}{|c|c|c|c|c|c|c|}
\hline $\begin{array}{c}\text { eak } \\
\#\end{array}$ & $\begin{array}{c}\text { RetTime } \\
\text { [min] }\end{array}$ & Type & $\begin{array}{l}\text { Width } \\
\text { [min] }\end{array}$ & $\begin{array}{c}\text { Area } \\
{\left[\mathrm{mAU}^{\star} \mathrm{s}\right]}\end{array}$ & $\begin{array}{l}\text { Height } \\
{[\mathrm{mAU}]}\end{array}$ & $\begin{array}{c}\text { Area } \\
\frac{\circ}{0}\end{array}$ \\
\hline & & & & & -- & \\
\hline & & & & $4 e 4$ & 920 & 52 \\
\hline & & & 28 & $2.23023 \mathrm{e} 4$ & 766 & 448 \\
\hline
\end{tabular}


Table 4, entry 3:

Reaction in $95 / 5 \mathrm{MeCN} / \mathrm{H}_{2} \mathrm{O}$ at $20{ }^{\circ} \mathrm{C}$ for $12 \mathrm{~h}$

HPLC (OD-H column, eluent: 80/20 hexane/iPrOH, flow rate: $0.8 \mathrm{~mL} / \mathrm{min}$ )

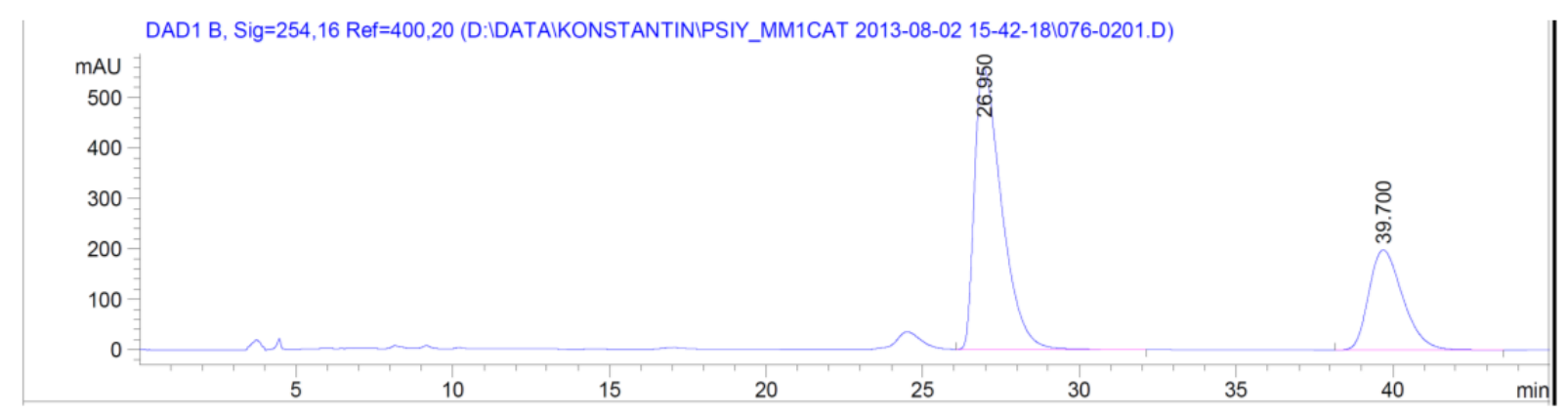

Signal 1: DAD1 B, Sig=254,16 Ref=400,20

\begin{tabular}{|c|c|c|c|c|c|c|}
\hline $\begin{array}{c}\text { Peak } \\
\quad \#\end{array}$ & $\begin{array}{c}\text { RetTime } \\
\text { [min] }\end{array}$ & Type & $\begin{array}{l}\text { Width } \\
\text { [min] }\end{array}$ & $\begin{array}{c}\text { Area } \\
{\left[\mathrm{mAU}{ }^{\star} \mathrm{s}\right]}\end{array}$ & $\begin{array}{l}\text { Height } \\
{[\mathrm{mAU}]}\end{array}$ & $\begin{array}{c}\text { Area } \\
\frac{\circ}{\partial}\end{array}$ \\
\hline$--\gamma_{1}$ & te- & & --- & ---- & --- & -- \\
\hline 1 & 26.950 & $B B$ & 0.9149 & $3.35447 e 4$ & 558.39197 & 69.1068 \\
\hline 2 & 39.700 & BB & 1.1651 & $1.49957 \mathrm{e} 4$ & 198.54712 & 8932 \\
\hline
\end{tabular}

Table 4, entry 4:

Reaction in $90 / 10 \mathrm{MeCN} / \mathrm{H}_{2} \mathrm{O}$ at $20{ }^{\circ} \mathrm{C}$ for $12 \mathrm{~h}$

HPLC ( OD-H column, eluent: 80/20 hexane/iPrOH, flow rate: $0.8 \mathrm{~mL} / \mathrm{min}$ )

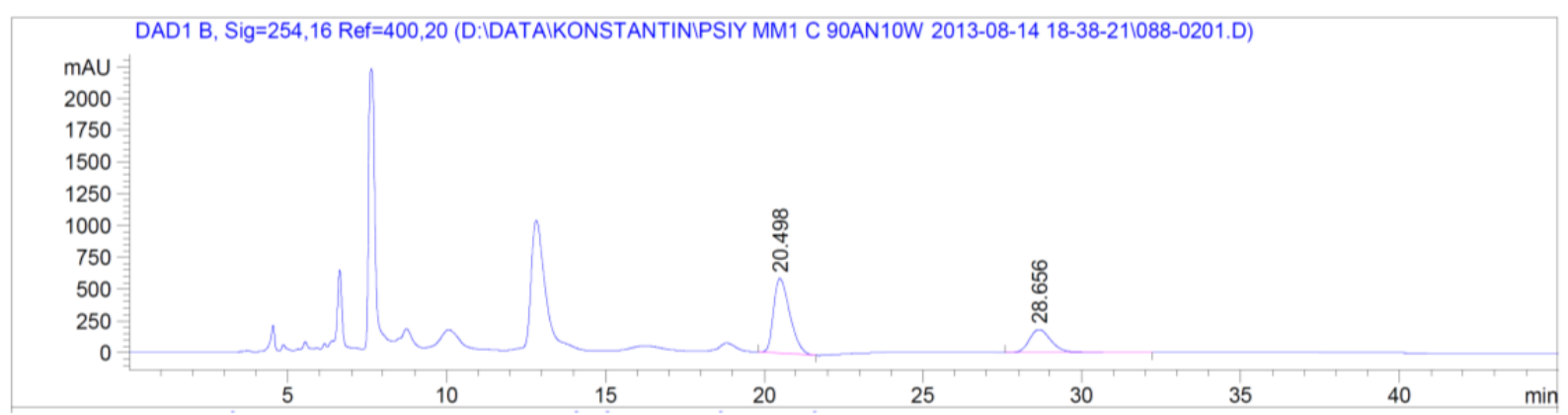

Signal 1: DAD1 B, Sig=254,16 Ref=400,20

\begin{tabular}{|c|c|c|c|c|c|c|}
\hline $\begin{array}{c}\text { Peak } \\
\#\end{array}$ & $\begin{array}{c}\text { RetTime } \\
\text { [min] }\end{array}$ & Type & $\begin{array}{l}\text { Width } \\
\text { [min] }\end{array}$ & $\begin{array}{c}\text { Area } \\
{\left[\mathrm{mAU}{ }^{\star} \mathrm{s}\right]}\end{array}$ & $\begin{array}{l}\text { Height } \\
\text { [mAU] }\end{array}$ & $\begin{array}{c}\text { Area } \\
\frac{\circ}{0}\end{array}$ \\
\hline $5-1$ & --- & & --- & ------ & ------ & $--------\mid$ \\
\hline 1 & 20.498 & BB & 0.5512 & $2.08831 e 4$ & 586.74017 & 70.8806 \\
\hline 2 & 28.656 & BB & 0.7322 & 8579.25488 & 181.26718 & 29.1194 \\
\hline
\end{tabular}


Table 4, entry 5:

Reaction in $80 / 20 \mathrm{MeCN} / \mathrm{H}_{2} \mathrm{O}$ at $20{ }^{\circ} \mathrm{C}$ for $12 \mathrm{~h}$

HPLC (OD-H column, eluent: 80/20 hexane/iPrOH, flow rate: $0.8 \mathrm{~mL} / \mathrm{min}$ )

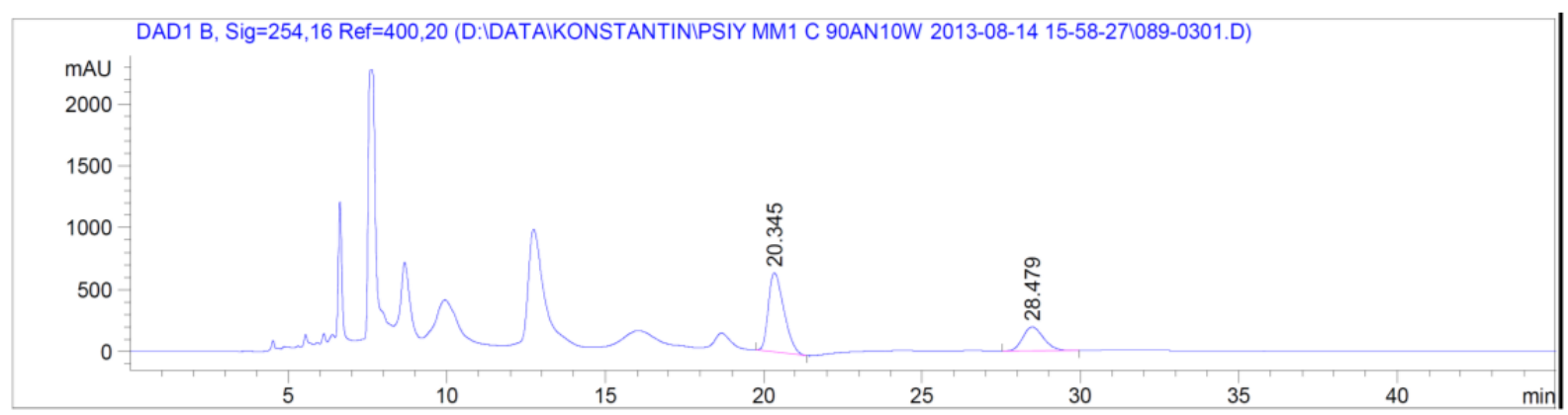

Signal 1: DAD1 B, Sig=254,16 Ref=400,20

\begin{tabular}{|c|c|c|c|c|c|c|}
\hline $\begin{array}{c}\text { Peak } \\
\#\end{array}$ & $\begin{array}{c}\text { RetTime } \\
\text { [min] }\end{array}$ & Type & $\begin{array}{l}\text { Width } \\
\text { [min] }\end{array}$ & $\begin{array}{c}\text { Area } \\
{\left[\mathrm{mAU}{ }^{*} \mathrm{~s}\right]}\end{array}$ & $\begin{array}{l}\text { Height } \\
{[\mathrm{mAU}]}\end{array}$ & $\begin{array}{c}\text { Area } \\
\frac{\circ}{0}\end{array}$ \\
\hline-1 & ----- & -1 & ------- & $----------\mid$ & $\cdot---------$ & $-------\mid$ \\
\hline 1 & 20.345 & BB & 0.5288 & $2.18329 \mathrm{e} 4$ & 638.78290 & 71.5060 \\
\hline 2 & 28.479 & $\mathrm{BB}$ & 0.7034 & 8700.06543 & 192.45691 & 28.4940 \\
\hline
\end{tabular}

Table 4, entry 6:

Reaction in $\mathrm{MeCN}$ at $-30{ }^{\circ} \mathrm{C}$ for $12 \mathrm{~h}$

HPLC (OD-H column, eluent: 80/20 hexane/iPrOH, flow rate: $0.8 \mathrm{~mL} / \mathrm{min}$ )
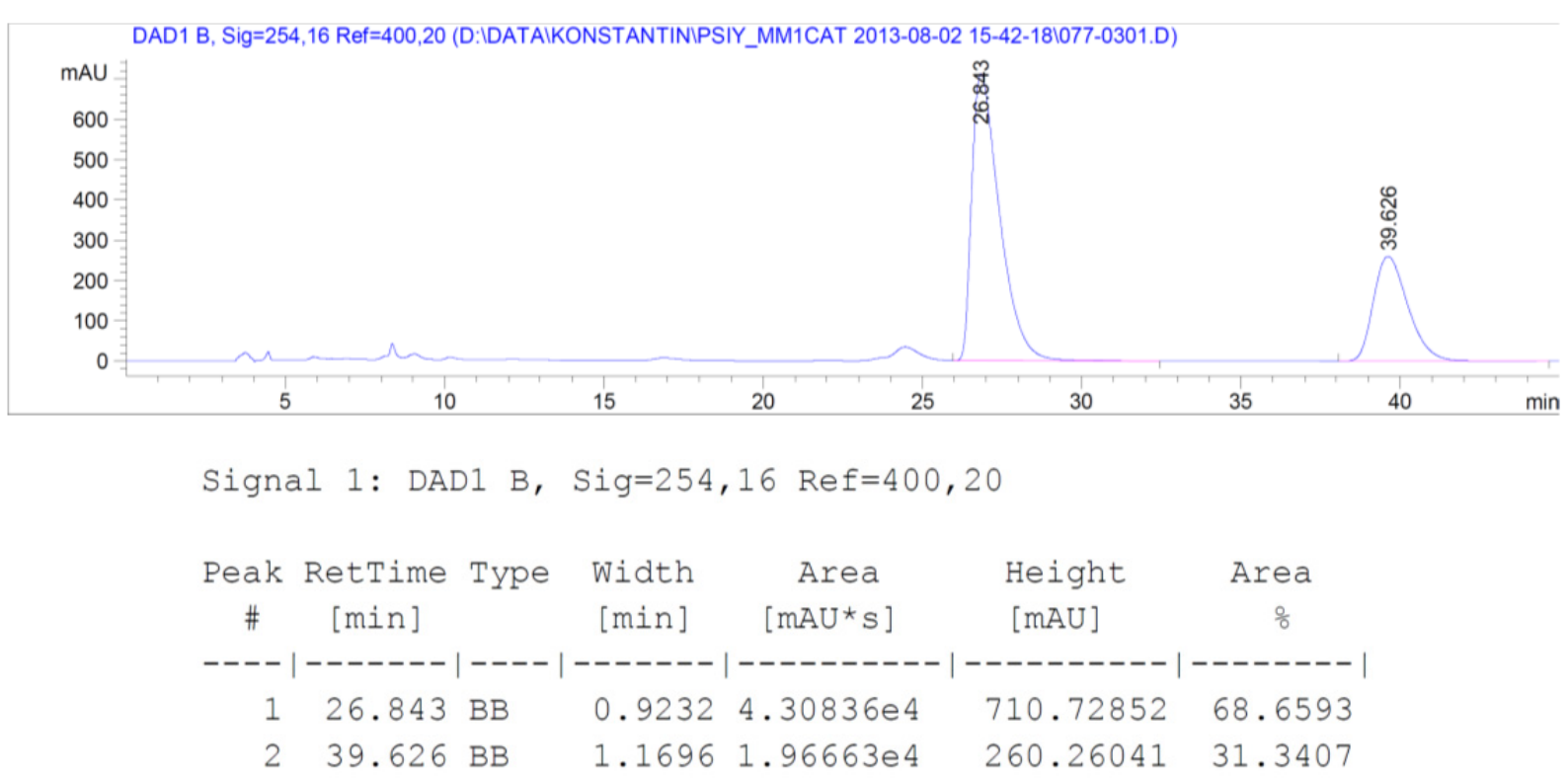
Table 4, entry 7:

Reaction in $95 / 5 \mathrm{MeCN} / \mathrm{H}_{2} \mathrm{O}$ at $0{ }^{\circ} \mathrm{C}$ for $12 \mathrm{~h}$

HPLC (OD-H column, eluent: 80/20 hexane/iPrOH, flow rate: $0.8 \mathrm{~mL} / \mathrm{min}$ )

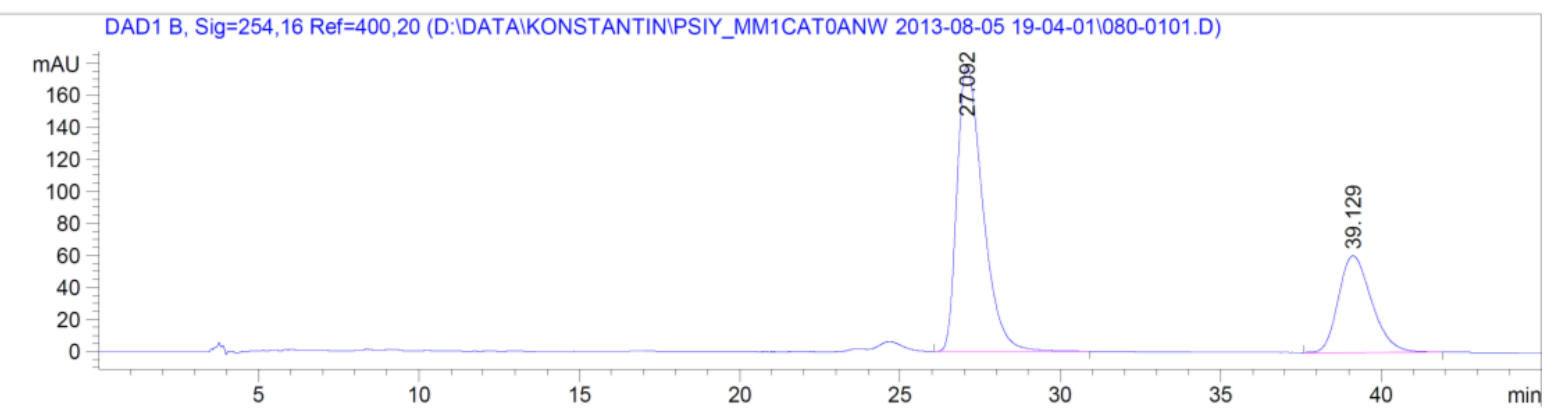

Signal 1: DAD1 B, Sig=254,16 Ref=400,20

\begin{tabular}{|c|c|c|c|c|c|c|}
\hline $\begin{array}{c}\text { Peak } \\
\quad \#\end{array}$ & $\begin{array}{c}\text { RetTime } \\
\text { [min] }\end{array}$ & Type & $\begin{array}{l}\text { Width } \\
\text { [min] }\end{array}$ & $\begin{array}{c}\text { Area } \\
{\left[\mathrm{mAU}{ }^{\star} \mathrm{s}\right]}\end{array}$ & $\begin{array}{l}\text { Height } \\
{[\mathrm{mAU}]}\end{array}$ & $\begin{array}{c}\text { Area } \\
\frac{\circ}{\circ}\end{array}$ \\
\hline & & & & $----------\mid$ & $----------\mid$ & $--------\mid$ \\
\hline 1 & 27.092 & BB & 0.8560 & 9968.80078 & 178.37524 & 69.6948 \\
\hline 2 & 39.129 & $\mathrm{BB}$ & 1.1032 & 4334.70215 & 60.25984 & 30.3052 \\
\hline
\end{tabular}

Table 4, entry 8 :

Reaction in $95 / 5 \mathrm{MeCN} / \mathrm{H}_{2} \mathrm{O}$ at $-30{ }^{\circ} \mathrm{C}$ for $12 \mathrm{~h}$

HPLC (OD-H column, eluent: 80/20 hexane/iPrOH, flow rate: $0.8 \mathrm{~mL} / \mathrm{min}$ )
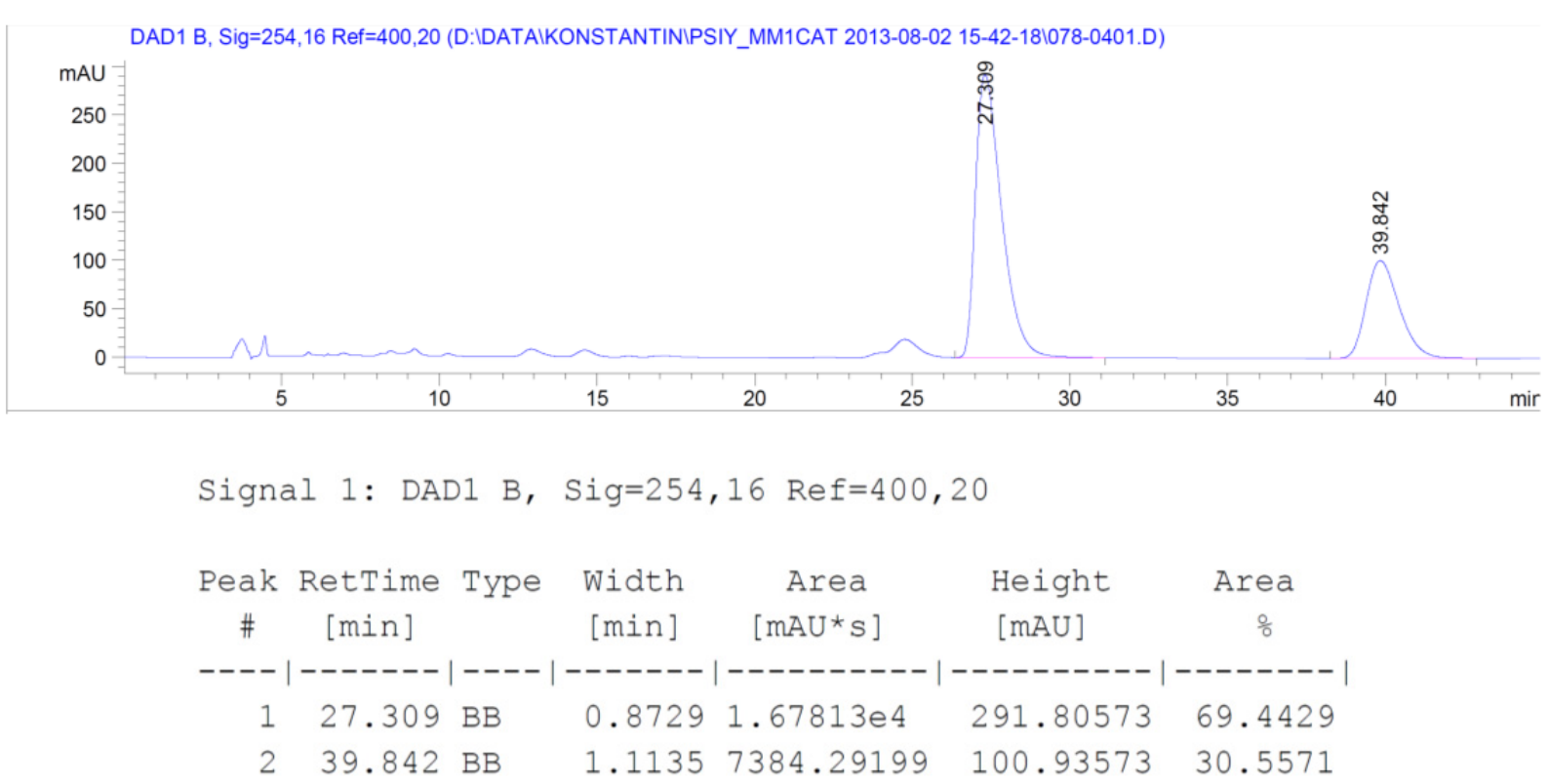
Table 4, entry 9:

Reaction in $95 / 5$ acetone $/ \mathrm{H}_{2} \mathrm{O}$ at $20{ }^{\circ} \mathrm{C}$ for $68 \mathrm{~h}$

HPLC (OD-H column, eluent: 80/20 hexane/iPrOH, flow rate: $0.8 \mathrm{~mL} / \mathrm{min}$ )

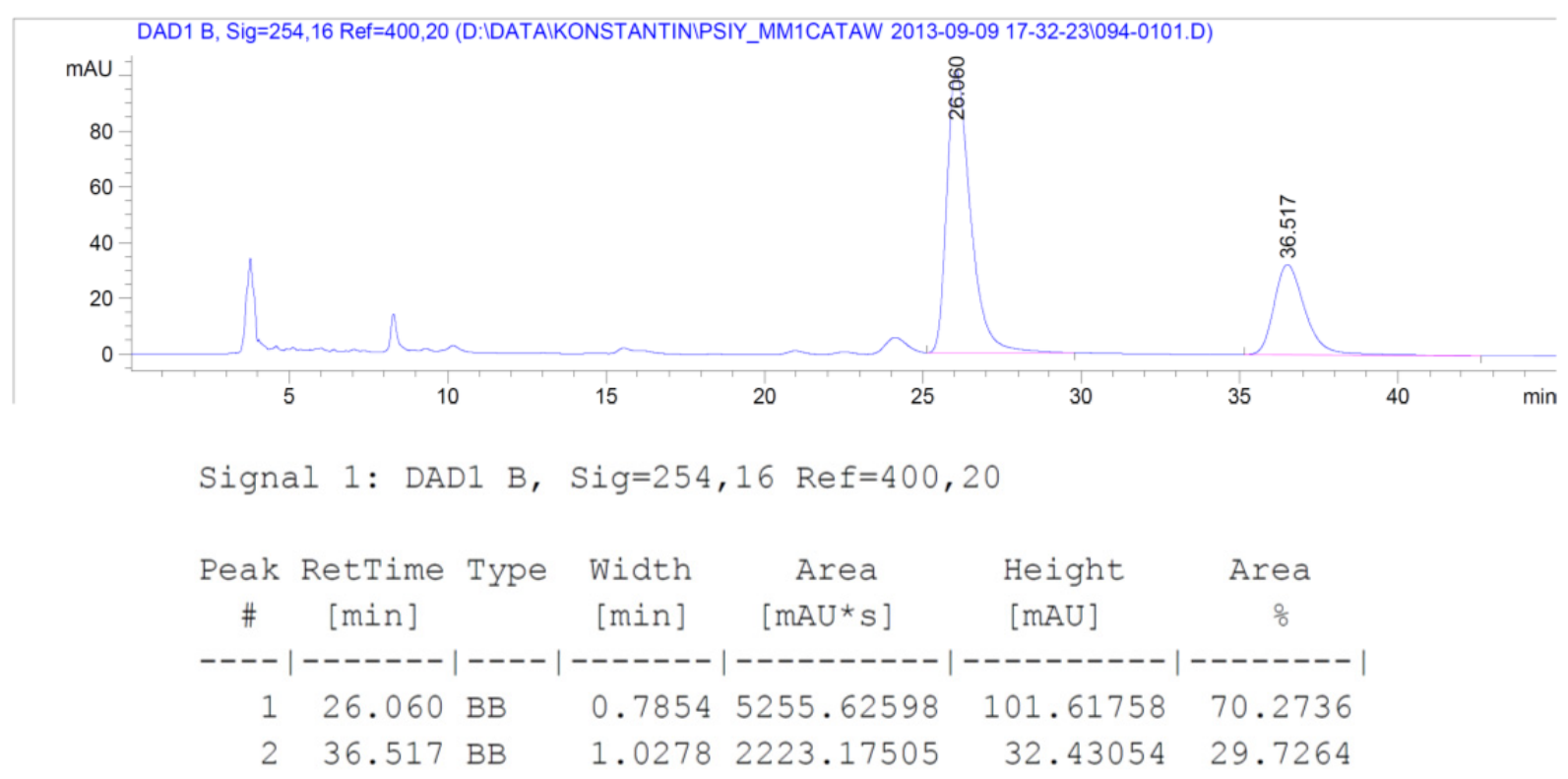


Reaction of the iodonium ylide 1 a with $\mathbf{1 0 b}-\mathbf{P F}_{\mathbf{6}}$ (Table 4, entry 10)

$(2 R, 5 R)-\mathbf{1 0 b}-\mathrm{PF}_{6}(212 \mathrm{mg}, 0.419 \mathrm{mmol})$ and $\mathbf{1 a}(147 \mathrm{mg}, 0.430 \mathrm{mmol})$ were dissolved in $\mathrm{MeCN}$. The resulting mixture was stirred for $2 \mathrm{~h}$ and then worked up as described above for 10a-PF 612 (32.1 mg, $0.132 \mathrm{mmol}, 32 \%)$.

Reaction in $\mathrm{MeCN}$ at $20^{\circ} \mathrm{C}$ for $2 \mathrm{~h}$

HPLC (OD-H column, eluent: 80/20 heptane/iPrOH, flow rate: $1.0 \mathrm{~mL} / \mathrm{min}$ )

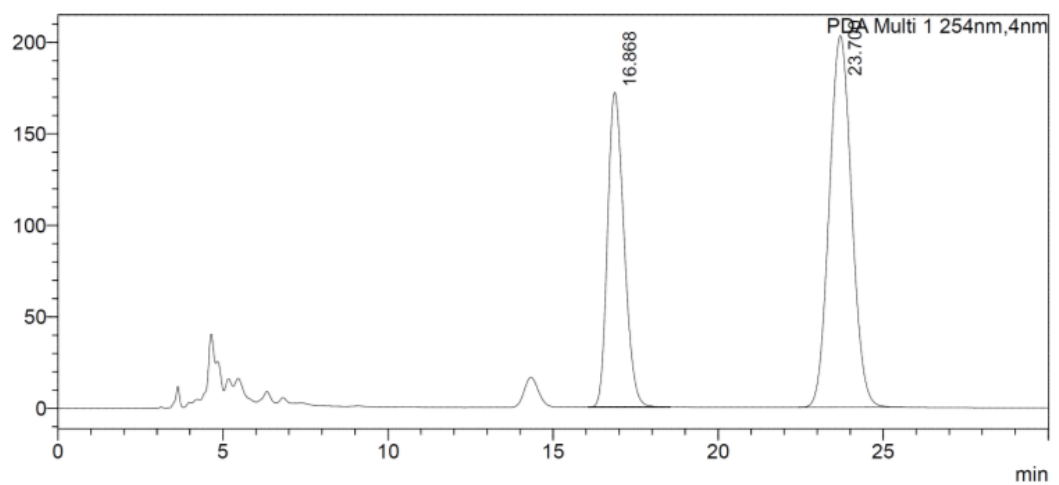

\begin{tabular}{|r|r|r|r|r|}
\hline Peak\# & Ret. Time & Area & Height & Conc. \\
\hline 1 & 16.868 & 5978680 & 172146 & 38.372 \\
\hline 2 & 23.700 & 9602201 & 203120 & 61.628 \\
\hline Total & & 15580881 & 375266 & \\
\hline
\end{tabular}


Reaction of the iodonium ylide 1 a with $\mathbf{1 0 b}-\mathbf{P F}_{\mathbf{6}}$ (Table 4, entry 11)

$(2 R, 5 R)-\mathbf{1 0 b}-\mathbf{P F}_{\mathbf{6}}(86 \mathrm{mg}, 170 \mu \mathrm{mol})$ and $\mathbf{1 a}(60 \mathrm{mg}, 170 \mu \mathrm{mol})$ were dissolved in MeCN. The resulting mixture was stirred for $0.1 \mathrm{~h}$. Then an aliquot from the reaction solution was analyzed by HPLC.

Reaction in $\mathrm{MeCN}$ at $20^{\circ} \mathrm{C}$ for $0.1 \mathrm{~h}$

HPLC (OD-H column, eluent: 80/20 heptane/iPrOH, flow rate: $1.0 \mathrm{~mL} / \mathrm{min}$ )

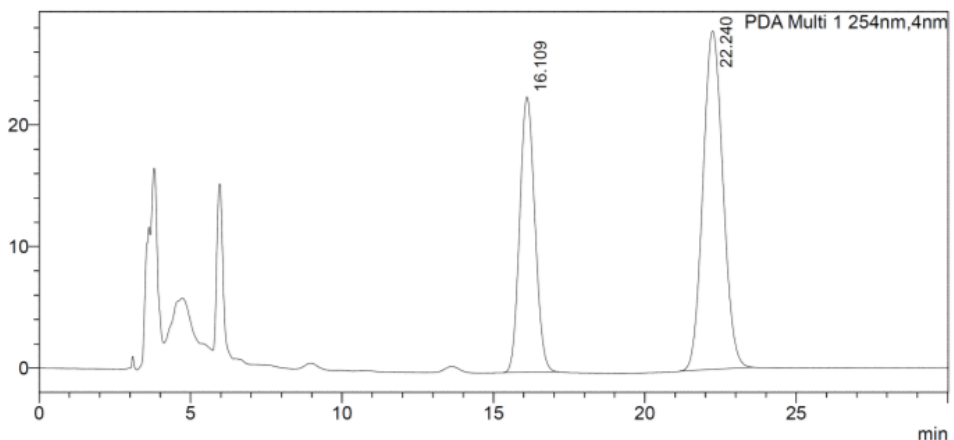

\begin{tabular}{|c|c|c|c|c|}
\hline \multirow{2}{*}{\multicolumn{2}{|c|}{$\begin{array}{l}\text { PDA Ch1 254nm } \\
\text { Peak\# Ret. Time }\end{array}$}} & & & \\
\hline & & Area & Height & Conc. \\
\hline 1 & 16.109 & 789195 & 22655 & 38.160 \\
\hline 2 & 22.240 & 1278913 & 27854 & 61.840 \\
\hline Total & & 2068107 & 50510 & \\
\hline
\end{tabular}


Reaction of the iodonium ylide 1 a with $\mathbf{1 0 b}-\mathbf{P F}_{\mathbf{6}}$ (Table 4, entry 12)

$(2 R, 5 R)-\mathbf{1 0 b}-\mathbf{P F}_{\mathbf{6}}(86 \mathrm{mg}, 170 \mu \mathrm{mol})$ and $\mathbf{1 a}(60 \mathrm{mg}, 170 \mu \mathrm{mol})$ were dissolved in $\mathrm{MeCN}$ at $-30{ }^{\circ} \mathrm{C}$. The resulting mixture was stirred for $12 \mathrm{~h}$. Then an aliquot from the reaction solution was analyzed by HPLC.

Reaction in $\mathrm{MeCN}$ at $-30{ }^{\circ} \mathrm{C}$ for $12 \mathrm{~h}$

HPLC (OD-H column, eluent: 80/20 heptane/iPrOH, flow rate: $1.0 \mathrm{~mL} / \mathrm{min}$ )

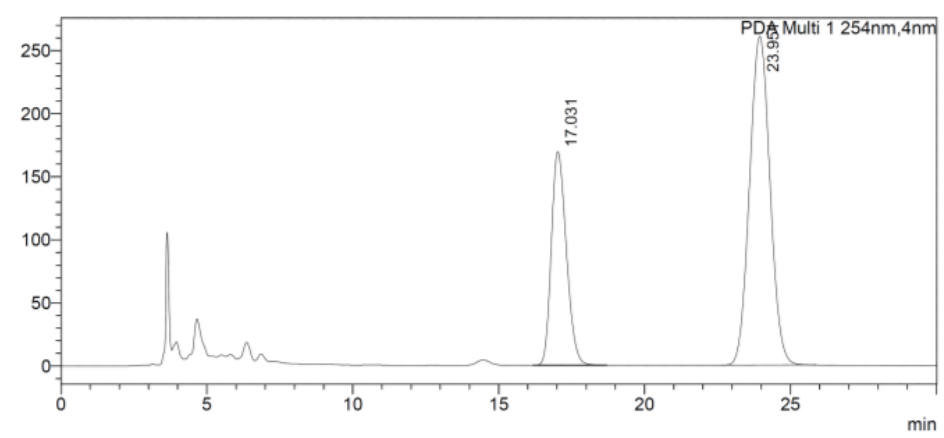

\begin{tabular}{|r|r|r|r|r|}
\hline Peak\# & Ret. Time & \multicolumn{1}{|c|}{ Area } & Height & Conc. \\
\hline 1 & 17.031 & 6056809 & 169332 & 32.292 \\
\hline 2 & 23.951 & 12699678 & 260528 & 67.708 \\
\hline Total & & 18756487 & 429861 & \\
\hline
\end{tabular}


Reaction of the iodonium ylide $1 \mathrm{a}$ with aldehyde 14 in the presence of $(2 R, 5 R)-11 \mathrm{~b}-\mathrm{PF}_{6}$

(Table 4, entry 13)

11b-PF 6 (31 mg, $79 \mu \mathrm{mol})$, aldehyde 14 (53.3 mg, $390 \mu \mathrm{mol})$, and 1a (136.5 mg, $390 \mu \mathrm{mol})$ were dissolved in $\mathrm{MeCN}$. The resulting mixture was stirred for $12 \mathrm{~h}$. Then an aliquot from the reaction solution was analyzed by HPLC.

Reaction in $\mathrm{MeCN}$ at $20^{\circ} \mathrm{C}$ for $12 \mathrm{~h}$

HPLC (OD-H column, eluent: 80/20 heptane/iPrOH, flow rate: $1.0 \mathrm{~mL} / \mathrm{min}$ )

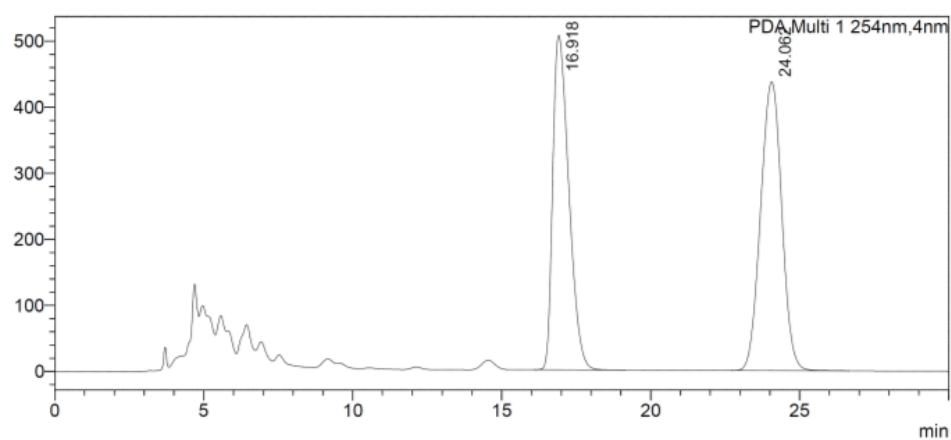

\begin{tabular}{|r|r|r|r|r|}
\hline Peak\# & Ret. Time & Area & Height & Conc. \\
\hline 1 & 16.918 & 18991372 & 506257 & 46.834 \\
\hline 2 & 24.062 & 21558653 & 437376 & 53.166 \\
\hline Total & & 40550025 & 943633 & \\
\hline
\end{tabular}


Reaction of the iodonium ylide 1 a with aldehyde 14 in the presence of $(2 R, 5 R)-11 \mathrm{~b}-\mathrm{PF}_{6}$

(Table 4, entry 14)

11b-PF 6 (31 mg, $79 \mu \mathrm{mol})$, aldehyde $14(53.3 \mathrm{mg}, 390 \mu \mathrm{mol})$, and 1a (136.5 mg, $390 \mu \mathrm{mol})$ were dissolved in a 95/5 MeCN/water mixture. The resulting mixture was stirred for $12 \mathrm{~h}$. Then an aliquot from the reaction solution was analyzed by HPLC.

Reaction in $95 / 5 \mathrm{MeCN} / \mathrm{H}_{2} \mathrm{O}$ at $20{ }^{\circ} \mathrm{C}$ for $12 \mathrm{~h}$

HPLC (OD-H column, eluent: $80 / 20$ hexane/iPrOH, flow rate: $0.8 \mathrm{~mL} / \mathrm{min}$ )

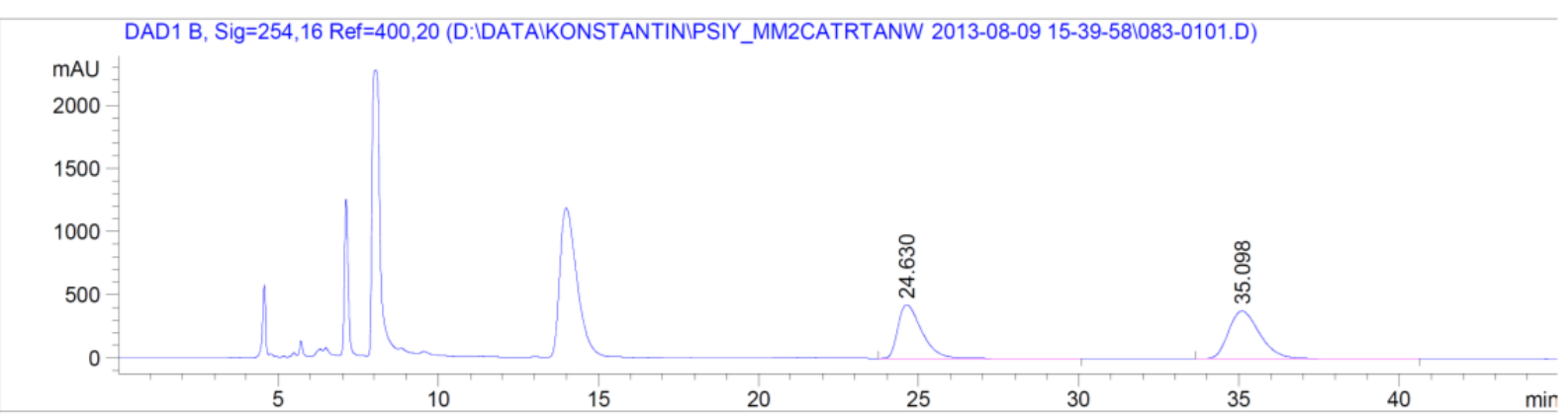

Signal 1: DAD1 B, Sig=254,16 Ref=400,20

\begin{tabular}{|c|c|c|c|c|c|c|}
\hline $\begin{array}{c}\text { Peak } \\
\#\end{array}$ & $\begin{array}{c}\text { RetTime } \\
\text { [min] }\end{array}$ & Type & $\begin{array}{l}\text { Width } \\
\text { [min] }\end{array}$ & $\begin{array}{c}\text { Area } \\
{\left[\mathrm{mAU}{ }^{\star} \mathrm{s}\right]}\end{array}$ & $\begin{array}{l}\text { Height } \\
\text { [mAU] }\end{array}$ & $\begin{array}{c}\text { Area } \\
\frac{\circ}{0}\end{array}$ \\
\hline$=-$ & -- & & - & $\mid---------$ & ---------- & $--------\mid$ \\
\hline 1 & 24.630 & $\mathrm{BB}$ & 0.7880 & $2.18933 \mathrm{e} 4$ & 425.69009 & 46.1514 \\
\hline 2 & 35.098 & $\mathrm{BB}$ & 1.0503 & $2.55448 e 4$ & 377.08170 & 53.8486 \\
\hline
\end{tabular}




\section{Kinetics}

\subsection{Reactions of the iodonium ylide 1a}

Kinetics of the reaction of $\mathbf{1 a}$ with $\mathbf{2 b}$ in $\mathrm{CH}_{2} \mathrm{Cl}_{2}$ at $20{ }^{\circ} \mathrm{C}$ (stopped-flow UV-Vis spectrometer, $\lambda=534 \mathrm{~nm})$.

\begin{tabular}{ccc}
\hline$[\mathbf{2 b}]_{0} / \mathrm{M}$ & {$[\mathbf{1 a}] / \mathrm{M}$} & $k_{\mathrm{obs}} / \mathrm{s}^{-1}$ \\
\hline $1.42 \times 10^{-5}$ & $6.43 \times 10^{-4}$ & 6.78 \\
$1.42 \times 10^{-5}$ & $9.64 \times 10^{-4}$ & $1.13 \times 10^{1}$ \\
$1.42 \times 10^{-5}$ & $1.28 \times 10^{-3}$ & $1.34 \times 10^{1}$ \\
$1.42 \times 10^{-5}$ & $1.61 \times 10^{-3}$ & $1.80 \times 10^{1}$ \\
$1.42 \times 10^{-5}$ & $1.93 \times 10^{-3}$ & $2.07 \times 10^{1}$ \\
\hline \multicolumn{3}{c}{$k_{2}=1.07 \times 10^{4} \mathrm{M}^{-1} \mathrm{~s}^{-1}$} \\
\hline
\end{tabular}

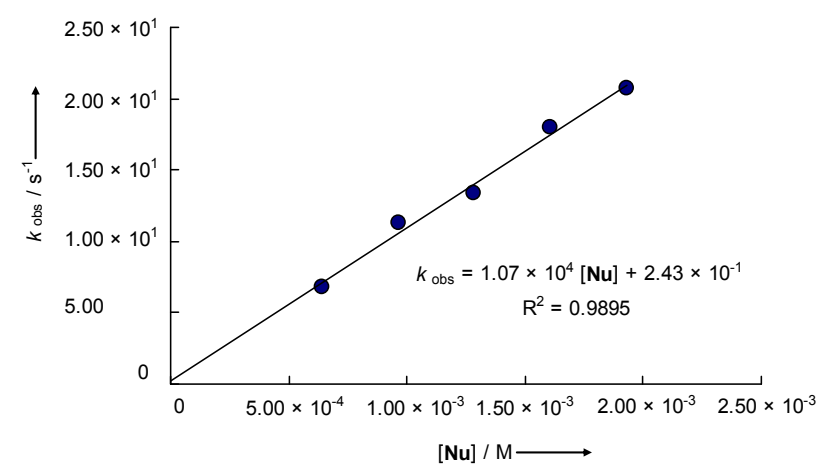

Kinetics of the reaction of $1 \mathbf{a}$ with $2 \mathbf{c}$ in $\mathrm{CH}_{2} \mathrm{Cl}_{2}$ at $20{ }^{\circ} \mathrm{C}$ (stopped-flow UV-Vis spectrometer, $\lambda=601 \mathrm{~nm})$.

\begin{tabular}{ccc}
\hline$[\mathbf{2 c}]_{0} / \mathrm{M}^{-1}$ & {$[\mathbf{1 a}] / \mathrm{M}^{-1}$} & $k_{\text {obs }} / \mathrm{s}^{-1}$ \\
\hline $1.98 \times 10^{-5}$ & $1.20 \times 10^{-3}$ & $2.69 \times 10^{-1}$ \\
$1.98 \times 10^{-5}$ & $1.60 \times 10^{-3}$ & $3.57 \times 10^{-1}$ \\
$1.98 \times 10^{-5}$ & $2.00 \times 10^{-3}$ & $4.47 \times 10^{-1}$ \\
$1.98 \times 10^{-5}$ & $2.40 \times 10^{-3}$ & $5.11 \times 10^{-1}$ \\
$1.98 \times 10^{-5}$ & $2.80 \times 10^{-3}$ & $6.16 \times 10^{-1}$ \\
\hline \multicolumn{3}{c}{$k_{2}=2.12 \times 10^{2} \mathrm{M}^{-1} \mathrm{~s}^{-1}$} \\
\hline
\end{tabular}

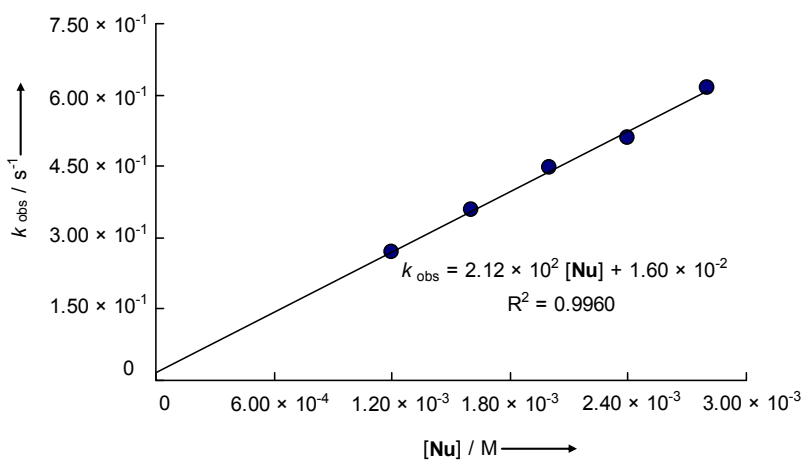

Kinetics of the reaction of $\mathbf{1 a}$ with $\mathbf{2 d}$ in $\mathrm{CH}_{2} \mathrm{Cl}_{2}$ at $20{ }^{\circ} \mathrm{C}$ (stopped-flow $\mathrm{UV}-\mathrm{V}$ is spectrometer, $\lambda=593 \mathrm{~nm})$.

\begin{tabular}{ccc}
\hline$[\mathbf{2 d}]_{0} / \mathrm{M}$ & {$[\mathbf{1 a}] / \mathrm{M}$} & $k_{\mathrm{obs}} / \mathrm{s}^{-1}$ \\
$1.25 \times 10^{-5}$ & $1.20 \times 10^{-3}$ & $9.27 \times 10^{-2}$ \\
$1.25 \times 10^{-5}$ & $1.60 \times 10^{-3}$ & $1.20 \times 10^{-1}$ \\
$1.25 \times 10^{-5}$ & $2.00 \times 10^{-3}$ & $1.45 \times 10^{-1}$ \\
$1.25 \times 10^{-5}$ & $2.40 \times 10^{-3}$ & $1.73 \times 10^{-1}$ \\
$1.25 \times 10^{-5}$ & $3.20 \times 10^{-3}$ & $2.27 \times 10^{-1}$ \\
$1.25 \times 10^{-6}$ & $3.60 \times 10^{-3}$ & $2.53 \times 10^{-1}$ \\
\hline \multicolumn{3}{c}{$k_{2}=6.69 \times 10^{1} \mathrm{M}^{-1} \mathrm{~s}^{-1}$}
\end{tabular}

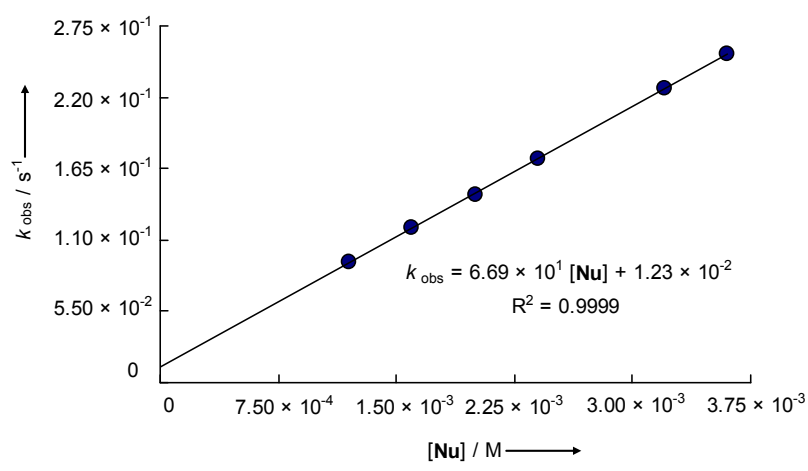


Kinetics of the reaction of $\mathbf{1 a}$ with $2 \mathbf{f}$ in $\mathrm{CH}_{2} \mathrm{Cl}_{2}$ at $20{ }^{\circ} \mathrm{C}$ (diode array $\mathrm{UV}-\mathrm{V}$ is spectrometer $\lambda$ $=620 \mathrm{~nm})$.

\begin{tabular}{ccc}
\hline$[\mathbf{2 f}]_{0} / \mathrm{M}^{1}$ & {$[\mathbf{1 a}] / \mathrm{M}^{-1}$} & $k_{\mathrm{obs}} / \mathrm{s}^{-1}$ \\
\hline $2.04 \times 10^{-5}$ & $3.74 \times 10^{-4}$ & $1.20 \times 10^{-3}$ \\
$2.04 \times 10^{-5}$ & $5.61 \times 10^{-4}$ & $1.86 \times 10^{-3}$ \\
$2.04 \times 10^{-5}$ & $7.48 \times 10^{-4}$ & $2.72 \times 10^{-3}$ \\
$2.04 \times 10^{-5}$ & $9.35 \times 10^{-4}$ & $3.59 \times 10^{-3}$ \\
\hline \multicolumn{3}{c}{$k_{2}=4.29 \mathrm{M}^{-1} \mathrm{~s}^{-1}$} \\
\hline
\end{tabular}

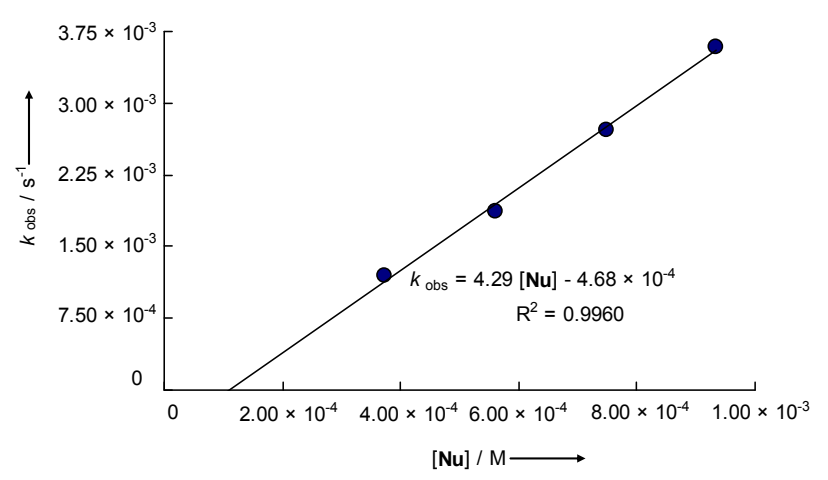

Kinetics of the reaction of $1 \mathrm{a}$ with $10 \mathrm{a}$ in $\mathrm{CH}_{2} \mathrm{Cl}_{2}$ at $20{ }^{\circ} \mathrm{C}$ (diode array $\mathrm{UV}-\mathrm{V}$ is spectrometer $\lambda=620 \mathrm{~nm})$.

\begin{tabular}{ccc} 
& & \\
\hline$[\mathbf{1 0 b}]_{0} / \mathrm{M}^{1}$ & {$[\mathbf{1 a}] / \mathrm{M}^{-1}$} & $k_{\mathrm{obs}} / \mathrm{s}^{-1}$ \\
\hline $3.63 \times 10^{-5}$ & $1.10 \times 10^{-3}$ & $3.81 \times 10^{-4}$ \\
$3.63 \times 10^{-5}$ & $1.47 \times 10^{-3}$ & $5.01 \times 10^{-4}$ \\
$3.63 \times 10^{-5}$ & $2.20 \times 10^{-3}$ & $6.43 \times 10^{-4}$ \\
\hline \multicolumn{3}{c}{$k_{2}=2.32 \times 10^{-1} \mathrm{M}^{-1} \mathrm{~s}^{-1}$} \\
\hline
\end{tabular}
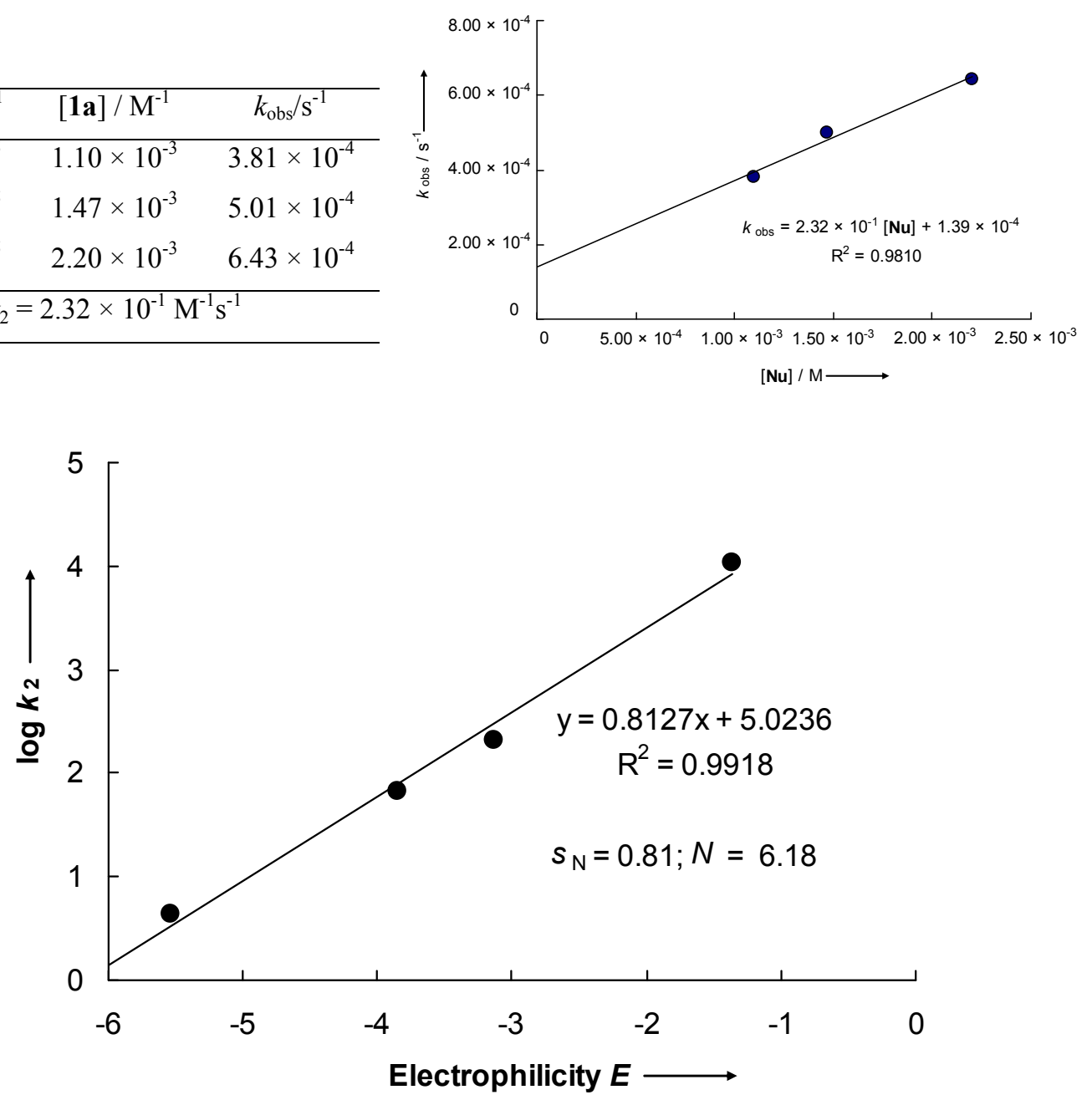

Figure S1. Determination of the nucleophilicity and sensitivity parameters $N$ and $s_{\mathrm{N}}$ of the iodonium ylide $\mathbf{1 a}$. 


\subsection{Reactions of the iodonium ylide $1 \mathrm{~b}$}

Kinetics of the reaction of $\mathbf{1 b}$ with $\mathbf{2 a}$ in $\mathrm{CH}_{2} \mathrm{Cl}_{2}$ at $20{ }^{\circ} \mathrm{C}$ (stopped-flow UV-Vis spectrometer, $\lambda=513 \mathrm{~nm})$.

\begin{tabular}{ccc}
\hline$[\mathbf{2 a}]_{0} / \mathrm{M}$ & {$[\mathbf{1 b}] / \mathrm{M}$} & $k_{\mathrm{obs}} / \mathrm{s}^{-1}$ \\
\hline $1.28 \times 10^{-5}$ & $9.79 \times 10^{-4}$ & $3.76 \times 10^{1}$ \\
$1.28 \times 10^{-5}$ & $1.31 \times 10^{-3}$ & $4.84 \times 10^{1}$ \\
$1.28 \times 10^{-5}$ & $1.63 \times 10^{-3}$ & $5.86 \times 10^{1}$ \\
$1.28 \times 10^{-5}$ & $1.95 \times 10^{-3}$ & $7.00 \times 10^{1}$ \\
\hline \multicolumn{3}{c}{$k_{2}=3.32 \times 10^{4} \mathrm{M}^{-1} \mathrm{~s}^{-1}$}
\end{tabular}

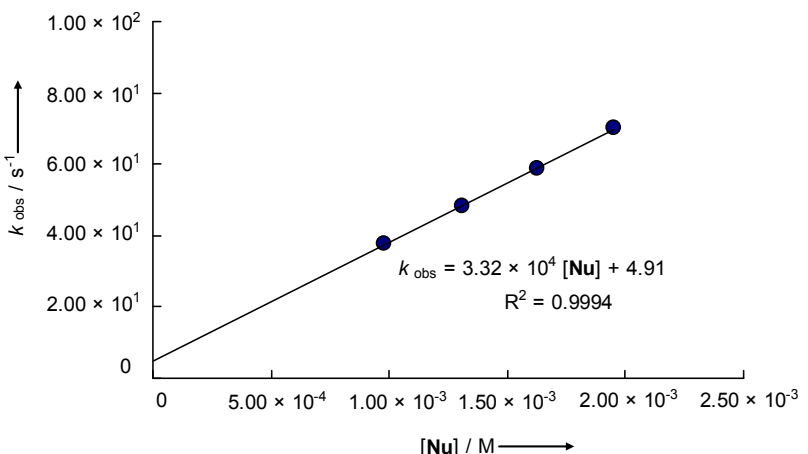

Kinetics of the reaction of $\mathbf{1 b}$ with $\mathbf{2 b}$ in $\mathrm{CH}_{2} \mathrm{Cl}_{2}$ at $20^{\circ} \mathrm{C}$ (stopped-flow $\mathrm{UV}$-Vis spectrometer, $\lambda=534 \mathrm{~nm}$ ).

\begin{tabular}{ccc}
\hline$[\mathbf{2 b}]_{0} / \mathrm{M}$ & {$[\mathbf{1 b}] / \mathrm{M}$} & $k_{\mathrm{obs}} / \mathrm{s}^{-1}$ \\
\hline $1.57 \times 10^{-5}$ & $5.95 \times 10^{-4}$ & $4.82 \times 10^{-1}$ \\
$1.57 \times 10^{-5}$ & $8.93 \times 10^{-4}$ & 1.12 \\
$1.57 \times 10^{-5}$ & $1.19 \times 10^{-3}$ & 1.71 \\
$1.57 \times 10^{-5}$ & $1.49 \times 10^{-3}$ & 2.29 \\
$1.57 \times 10^{-5}$ & $2.08 \times 10^{-3}$ & 3.66 \\
$1.57 \times 10^{-5}$ & $2.38 \times 10^{-3}$ & 4.48 \\
\hline \multicolumn{3}{c}{$k_{2}=2.21 \times 10^{3} \mathrm{M}^{-1} \mathrm{~s}^{-1}$}
\end{tabular}

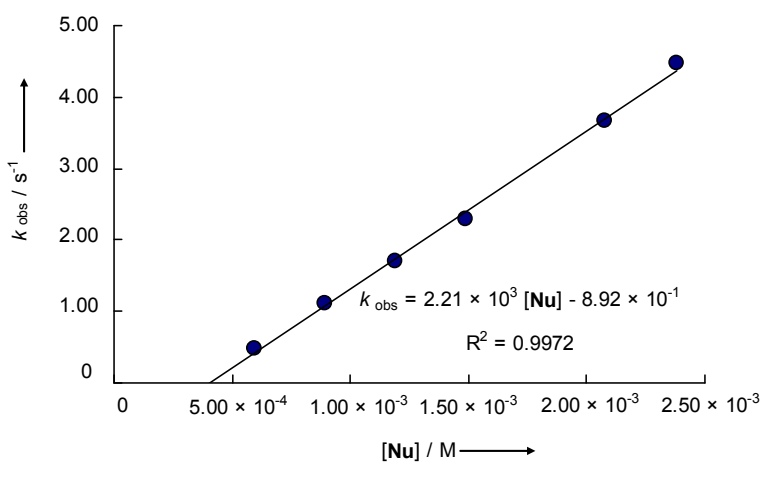

Kinetics of the reaction of $\mathbf{1 b}$ with $\mathbf{2} \mathbf{c}$ in $\mathrm{CH}_{2} \mathrm{Cl}_{2}$ at $20{ }^{\circ} \mathrm{C}$ (stopped-flow UV-Vis spectrometer, $\lambda=601 \mathrm{~nm})$.

\begin{tabular}{ccc}
\hline$[\mathbf{2 c}]_{0} / \mathrm{M}$ & {$[\mathbf{1 b}] / \mathrm{M}$} & $k_{\mathrm{obs}} / \mathrm{s}^{-1}$ \\
\hline $1.05 \times 10^{-5}$ & $3.32 \times 10^{-4}$ & $4.72 \times 10^{-3}$ \\
$1.05 \times 10^{-5}$ & $4.54 \times 10^{-4}$ & $6.24 \times 10^{-3}$ \\
$1.05 \times 10^{-5}$ & $5.14 \times 10^{-4}$ & $7.11 \times 10^{-3}$ \\
$1.05 \times 10^{-5}$ & $7.26 \times 10^{-4}$ & $1.01 \times 10^{-2}$ \\
$1.05 \times 10^{-5}$ & $9.08 \times 10^{-4}$ & $1.27 \times 10^{-2}$ \\
\hline \multicolumn{3}{c}{$k_{2}=1.40 \times 10^{1} \mathrm{M}^{-1} \mathrm{~s}^{-1}$}
\end{tabular}

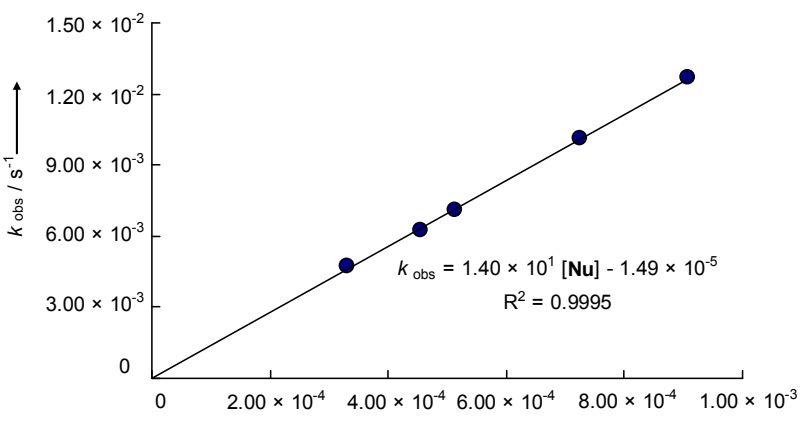

$[\mathrm{Nu}] / \mathrm{M} \longrightarrow$ 
Kinetics of the reaction of $\mathbf{1 b}$ with $\mathbf{2 d}$ in $\mathrm{CH}_{2} \mathrm{Cl}_{2}$ at $20{ }^{\circ} \mathrm{C}$ (stopped-flow UV-Vis spectrometer, $\lambda=601 \mathrm{~nm})$.

\begin{tabular}{ccc}
\hline$[\mathbf{2 d}]_{0} / \mathrm{M}$ & {$[\mathbf{1 b}] / \mathrm{M}$} & $k_{\mathrm{obs}} / \mathrm{s}^{-1}$ \\
\hline $1.95 \times 10^{-5}$ & $7.11 \times 10^{-4}$ & $2.93 \times 10^{-3}$ \\
$1.95 \times 10^{-5}$ & $8.91 \times 10^{-4}$ & $3.72 \times 10^{-3}$ \\
$1.95 \times 10^{-5}$ & $1.06 \times 10^{-3}$ & $4.24 \times 10^{-3}$ \\
$1.95 \times 10^{-5}$ & $1.24 \times 10^{-3}$ & $5.12 \times 10^{-3}$ \\
$1.95 \times 10^{-5}$ & $1.42 \times 10^{-3}$ & $5.72 \times 10^{-3}$ \\
\hline \multicolumn{3}{c}{$k_{2}=3.95 \mathrm{M}^{-1} \mathrm{~s}^{-1}$} \\
\hline
\end{tabular}
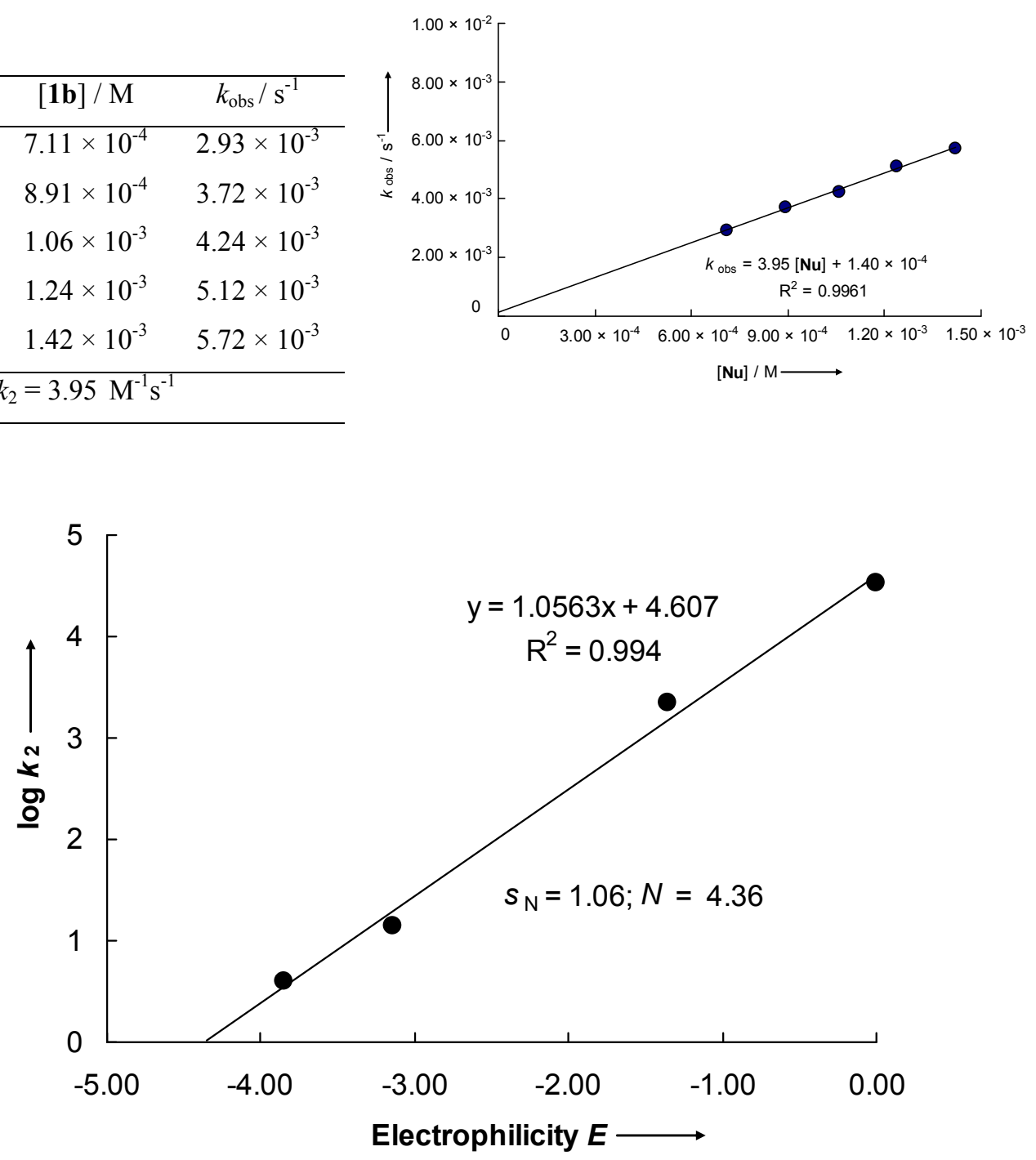

Figure S2. Determination of the nucleophilicity and sensitivity parameters $N$ and $s_{\mathrm{N}}$ of the iodonium ylide $\mathbf{1 b}$. 


\subsection{Reactions of the iodonium ylide 1c}

Kinetics of the reaction of $1 \mathbf{c}$ with $2 \mathrm{c}$ in $\mathrm{CH}_{2} \mathrm{Cl}_{2}$ at $20{ }^{\circ} \mathrm{C}$ (stopped-flow $\mathrm{UV}-\mathrm{Vis}$ spectrometer, $\lambda=601 \mathrm{~nm})$.

\begin{tabular}{ccc}
\hline$[\mathbf{2 d}]_{0} / \mathrm{M}$ & {$[\mathbf{1 c}] / \mathrm{M}$} & $k_{\mathrm{obs}} / \mathrm{s}^{-1}$ \\
\hline $3.90 \times 10^{-6}$ & $6.30 \times 10^{-4}$ & 2.03 \\
$3.90 \times 10^{-6}$ & $7.30 \times 10^{-4}$ & 2.19 \\
$3.90 \times 10^{-6}$ & $1.06 \times 10^{-3}$ & 3.28 \\
$3.90 \times 10^{-6}$ & $1.17 \times 10^{-3}$ & 3.47 \\
$3.90 \times 10^{-6}$ & $1.33 \times 10^{-3}$ & 4.17 \\
\hline \multicolumn{3}{c}{$k_{2}=3.04 \times 10^{3} \mathrm{M}^{-1} \mathrm{~s}^{-1}$}
\end{tabular}

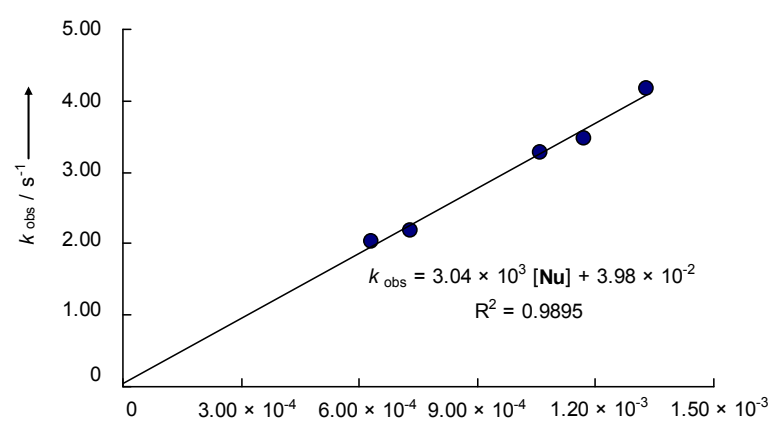

$[\mathrm{Nu}] / \mathrm{M} \longrightarrow$

Kinetics of the reaction of $\mathbf{1 c}$ with $\mathbf{2 d}$ in $\mathrm{CH}_{2} \mathrm{Cl}_{2}$ at $20{ }^{\circ} \mathrm{C}$ (stopped-flow UV-Vis spectrometer, $\lambda=593 \mathrm{~nm})$.

\begin{tabular}{ccc}
\hline$[\mathbf{2 d}]_{0} / \mathrm{M}$ & {$[\mathbf{1 c}] / \mathrm{M}$} & $k_{\mathrm{obs}} / \mathrm{s}^{-1}$ \\
\hline $5.20 \times 10^{-6}$ & $8.66 \times 10^{-4}$ & $6.27 \times 10^{-1}$ \\
$5.20 \times 10^{-6}$ & $1.30 \times 10^{-3}$ & $9.90 \times 10^{-1}$ \\
$5.20 \times 10^{-6}$ & $1.56 \times 10^{-3}$ & 1.10 \\
$5.20 \times 10^{-6}$ & $2.20 \times 10^{-3}$ & 1.55 \\
$5.20 \times 10^{-6}$ & $2.47 \times 10^{-3}$ & 1.62 \\
\hline \multicolumn{3}{c}{$k_{2}=6.25 \times 10^{2} \mathrm{M}^{-1} \mathrm{~s}^{-1}$}
\end{tabular}

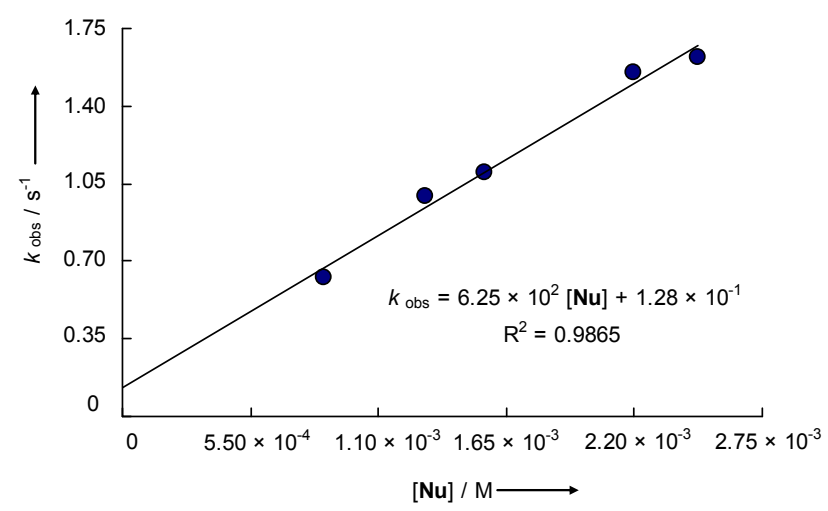

Kinetics of the reaction of $\mathbf{1 c}$ with $2 \mathbf{e}$ in $\mathrm{CH}_{2} \mathrm{Cl}_{2}$ at $20{ }^{\circ} \mathrm{C}$ (stopped-flow $\mathrm{UV}$-Vis spectrometer, $\lambda=672 \mathrm{~nm})$.

\begin{tabular}{ccc}
\hline$[\mathbf{2 e}]_{0} / \mathrm{M}$ & {$[\mathbf{1 c}] / \mathrm{M}$} & $k_{\mathrm{obs}} / \mathrm{s}^{-1}$ \\
\hline $8.37 \times 10^{-6}$ & $6.10 \times 10^{-4}$ & $1.27 \times 10^{-1}$ \\
$8.37 \times 10^{-6}$ & $7.50 \times 10^{-4}$ & $1.55 \times 10^{-1}$ \\
$8.37 \times 10^{-6}$ & $9.60 \times 10^{-4}$ & $2.06 \times 10^{-1}$ \\
$8.37 \times 10^{-6}$ & $1.01 \times 10^{-3}$ & $2.25 \times 10^{-1}$ \\
$8.37 \times 10^{-6}$ & $1.50 \times 10^{-3}$ & $3.27 \times 10^{-1}$ \\
\hline \multicolumn{3}{c}{$k_{2}=2.27 \times 10^{2} \mathrm{M}^{-1} \mathrm{~s}^{-1}$}
\end{tabular}

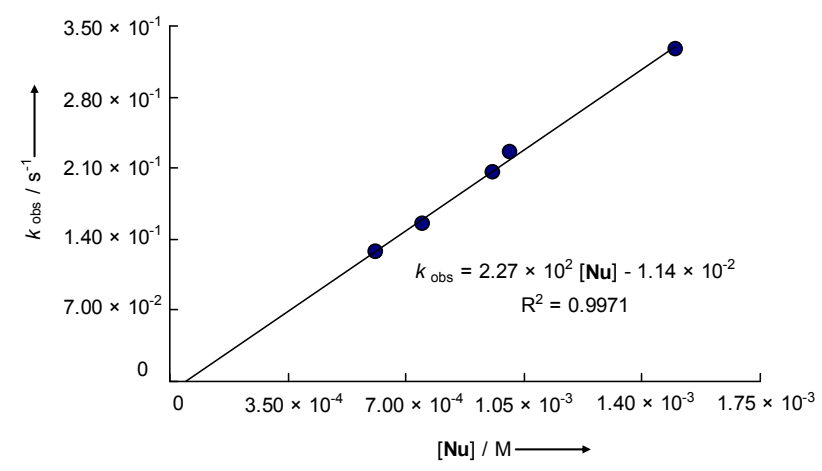




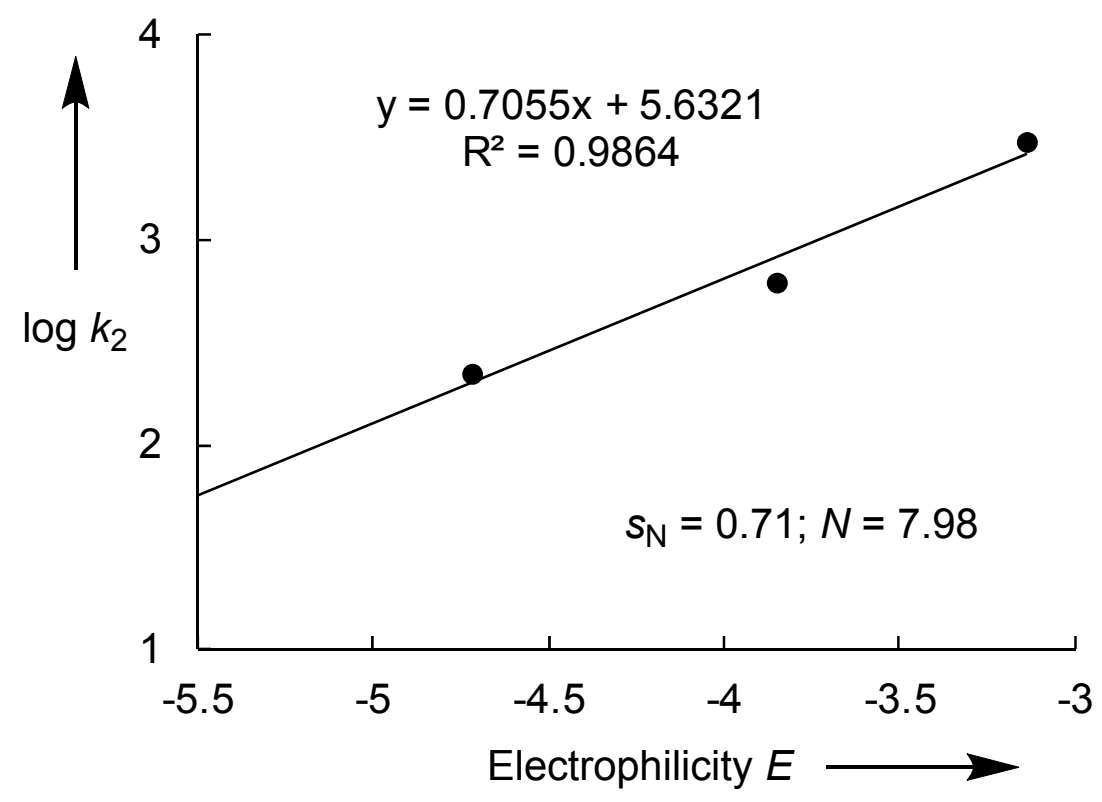

Figure S3. Determination of the nucleophilicity and sensitivity parameters $N$ and $s_{\mathrm{N}}$ of the iodonium ylide $\mathbf{1 c}$. 


\subsection{Reactions of the iodonium ylide 1d}

Kinetics of the reaction of $\mathbf{1 d}$ with $2 \mathbf{c}$ in $\mathrm{CH}_{2} \mathrm{Cl}_{2}$ at $20{ }^{\circ} \mathrm{C}$ (diode array UV-Vis spectrometer, $\lambda$ $=601 \mathrm{~nm})$.

\begin{tabular}{ccc}
\hline$[\mathbf{2 c}]_{0} / \mathrm{M}$ & {$[\mathbf{1 d}] / \mathrm{M}$} & $k_{\mathrm{obs}} / \mathrm{s}^{-1}$ \\
\hline $1.60 \times 10^{-5}$ & $7.27 \times 10^{-4}$ & $3.35 \times 10^{-2}$ \\
$1.60 \times 10^{-5}$ & $1.27 \times 10^{-3}$ & $4.82 \times 10^{-2}$ \\
$1.60 \times 10^{-5}$ & $1.51 \times 10^{-3}$ & $5.48 \times 10^{-2}$ \\
$1.60 \times 10^{-5}$ & $1.81 \times 10^{-3}$ & $6.14 \times 10^{-2}$ \\
\hline \multicolumn{3}{c}{$k_{2}=2.60 \times 10^{1} \mathrm{M}^{-1} \mathrm{~s}^{-1}$} \\
\hline
\end{tabular}

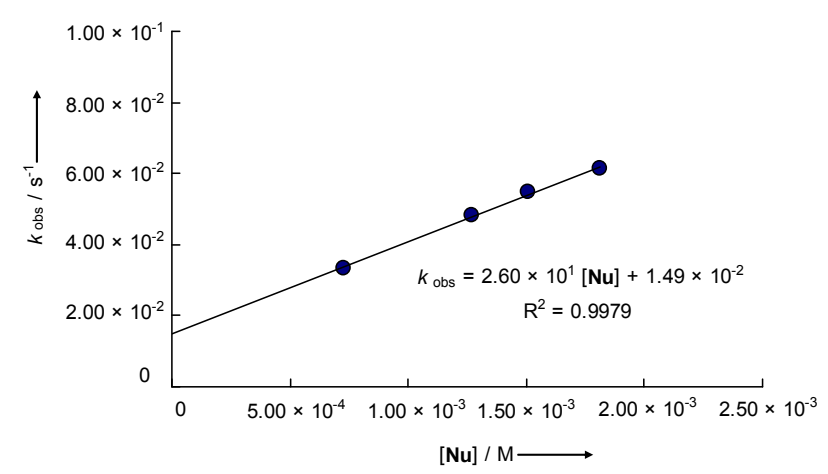

Kinetics of the reaction of $\mathbf{1 d}$ with $\mathbf{2 d}$ in $\mathrm{CH}_{2} \mathrm{Cl}_{2}$ at $20{ }^{\circ} \mathrm{C}$ (diode array $\mathrm{UV}-\mathrm{Vis}$ spectrometer, $\lambda=593 \mathrm{~nm})$.

\begin{tabular}{ccc}
\hline$[\mathbf{2 d}]_{0} / \mathrm{M}$ & {$[\mathbf{1 d}] / \mathrm{M}$} & $k_{\mathrm{obs}} / \mathrm{s}^{-1}$ \\
\hline $1.22 \times 10^{-5}$ & $5.80 \times 10^{-4}$ & $3.38 \times 10^{-3}$ \\
$1.22 \times 10^{-5}$ & $6.90 \times 10^{-4}$ & $4.19 \times 10^{-3}$ \\
$1.22 \times 10^{-5}$ & $8.10 \times 10^{-4}$ & $5.05 \times 10^{-3}$ \\
$1.22 \times 10^{-5}$ & $1.00 \times 10^{-3}$ & $6.55 \times 10^{-3}$ \\
\hline \multicolumn{3}{c}{$k_{2}=7.54 \mathrm{M}^{-1} \mathrm{~s}^{-1}$} \\
\hline
\end{tabular}

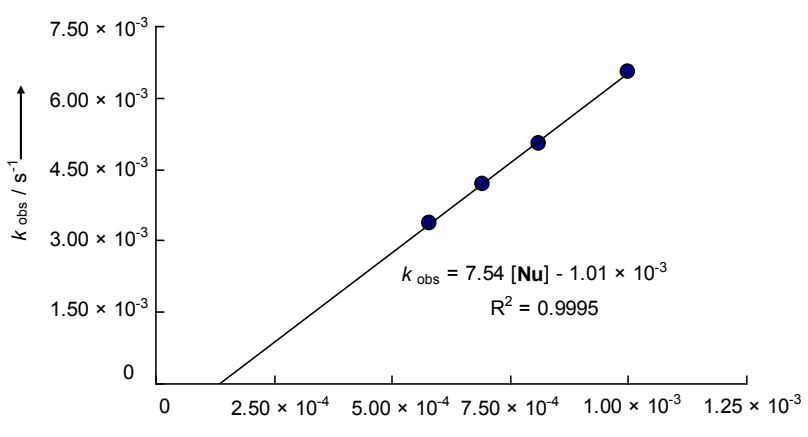

$[\mathrm{Nu}] / \mathrm{M} \longrightarrow$

Kinetics of the reaction of $\mathbf{1 d}$ with $2 \mathrm{e}$ in $\mathrm{CH}_{2} \mathrm{Cl}_{2}$ at $20{ }^{\circ} \mathrm{C}$ (diode array UV-Vis spectrometer, $\lambda$ $=674 \mathrm{~nm})$.

\begin{tabular}{ccc}
\hline$[\mathbf{2 e}]_{0} / \mathrm{M}$ & {$[\mathbf{1 d}] / \mathrm{M}$} & $k_{\text {obs }} / \mathrm{s}^{-1}$ \\
\hline $1.48 \times 10^{-5}$ & $7.48 \times 10^{-4}$ & $7.05 \times 10^{-4}$ \\
$1.48 \times 10^{-5}$ & $8.97 \times 10^{-4}$ & $8.03 \times 10^{-4}$ \\
$1.48 \times 10^{-5}$ & $1.07 \times 10^{-3}$ & $8.45 \times 10^{-4}$ \\
$1.48 \times 10^{-5}$ & $1.19 \times 10^{-3}$ & $9.41 \times 10^{-4}$ \\
\hline \multicolumn{3}{c}{$k_{2}=4.94 \times 10^{-1} \mathrm{M}^{-1} \mathrm{~s}^{-1}$}
\end{tabular}

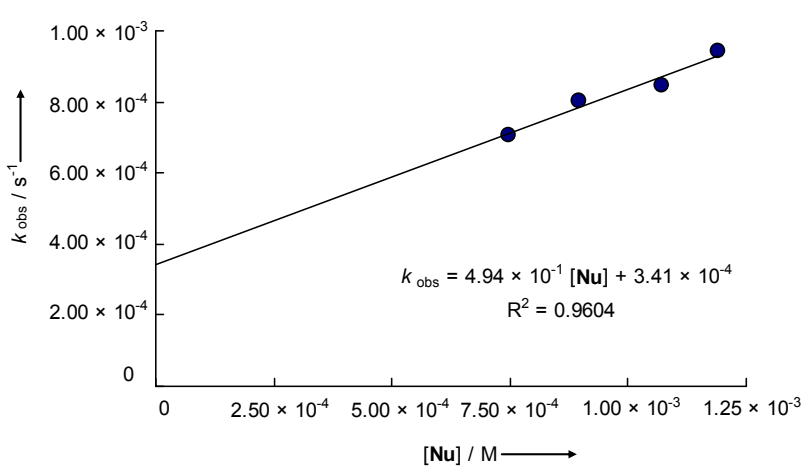


Kinetics of the reaction of $\mathbf{1 d}$ with $\mathbf{2 f}$ in $\mathrm{CH}_{2} \mathrm{Cl}_{2}$ at $20{ }^{\circ} \mathrm{C}$ (diode array UV-Vis spectrometer, $\lambda$ $=620 \mathrm{~nm})$.

\begin{tabular}{ccc}
\hline$[\mathbf{2 f}]_{0} / \mathrm{M}$ & {$[\mathbf{1 d}] / \mathrm{M}$} & $k_{\mathrm{obs}} / \mathrm{s}^{-1}$ \\
\hline $2.40 \times 10^{-5}$ & $4.56 \times 10^{-4}$ & $1.43 \times 10^{-4}$ \\
$2.40 \times 10^{-5}$ & $1.14 \times 10^{-3}$ & $2.88 \times 10^{-4}$ \\
$2.40 \times 10^{-5}$ & $1.36 \times 10^{-3}$ & $3.30 \times 10^{-4}$ \\
$2.40 \times 10^{-5}$ & $1.82 \times 10^{-3}$ & $4.42 \times 10^{-4}$ \\
\hline \multicolumn{3}{c}{$k_{2}=2.18 \times 10^{-1} \mathrm{M}^{-1} \mathrm{~s}^{-1}$}
\end{tabular}
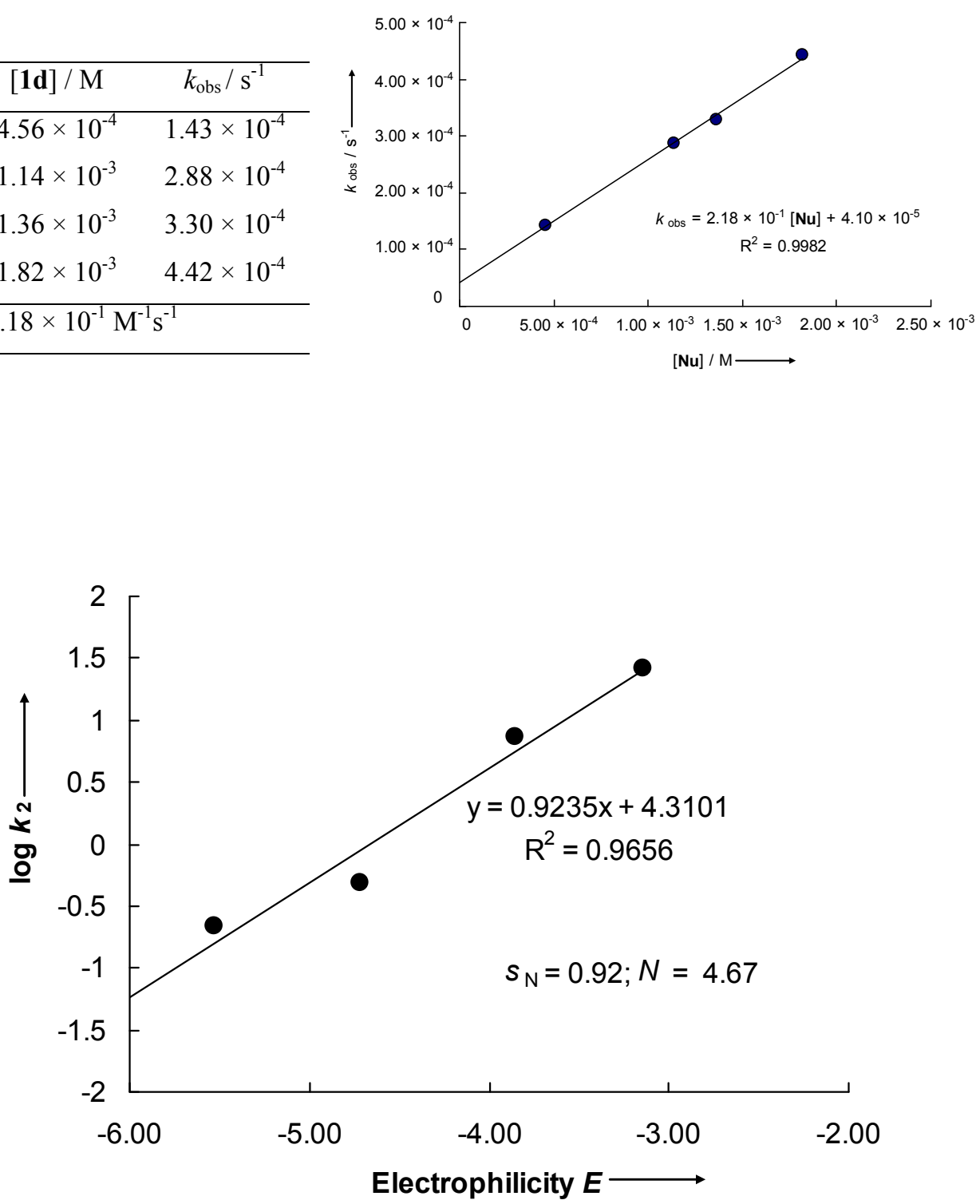

Figure S4. Determination of the nucleophilicity and sensitivity parameters $N$ and $s_{\mathrm{N}}$ of the iodonium ylide $\mathbf{1 d}$. 


\subsection{Reactions of the 6-methyl-2,4-dioxotetrahydro-2H-pyran-3-ide with electrophiles 2i-k in DMSO}

Kinetics of the reactions of potassium 6-methyl-2,4-dioxotetrahydro-2H-pyran-3-ide with carbocation $2 \mathbf{i}\left(2 \mathbf{i}-\mathrm{BF}_{4}\right)$ in DMSO at $20{ }^{\circ} \mathrm{C}$ (stopped-flow UV-Vis spectrometry, $\lambda=618 \mathrm{~nm}$ ).

\begin{tabular}{ccc}
\hline$[\mathbf{2 i}]_{0} / \mathrm{M}$ & {$[\mathbf{1 4}] / \mathrm{M}$} & $k_{\text {obs }} / \mathrm{s}^{-1}$ \\
\hline $3.29 \times 10^{-5}$ & $1.23 \times 10^{-3}$ & $8.22 \times 10^{1}$ \\
$3.29 \times 10^{-5}$ & $1.84 \times 10^{-3}$ & $1.33 \times 10^{2}$ \\
$3.29 \times 10^{-5}$ & $2.45 \times 10^{-3}$ & $1.82 \times 10^{2}$ \\
$3.29 \times 10^{-5}$ & $3.07 \times 10^{-3}$ & $2.19 \times 10^{2}$ \\
$3.29 \times 10^{-5}$ & $3.68 \times 10^{-3}$ & $2.53 \times 10^{2}$ \\
$3.29 \times 10^{-5}$ & $4.29 \times 10^{-3}$ & $2.92 \times 10^{2}$ \\
\hline \multicolumn{3}{c}{$k_{2}=6.74 \times 10^{4} \mathrm{M}^{-1} \mathrm{~s}^{-1}$}
\end{tabular}

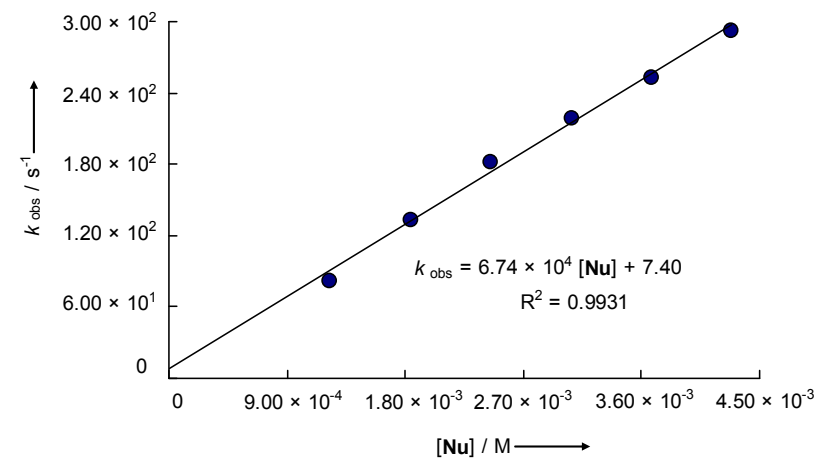

Kinetics of the reactions of potassium 6-methyl-2,4-dioxotetrahydro-2H-pyran-3-ide with carbocation $\mathbf{2} \mathbf{j}\left(\mathbf{2} \mathbf{j}-\mathrm{BF}_{4}\right)$ in DMSO at $20{ }^{\circ} \mathrm{C}$ (stopped-flow UV-Vis spectrometry, $\lambda=635 \mathrm{~nm}$ ).

\begin{tabular}{ccc}
\hline$[\mathbf{2} \mathbf{j}]_{0} / \mathrm{M}$ & {$[\mathbf{1 4}] / \mathrm{M}$} & $k_{\mathrm{obs}} / \mathrm{s}^{-1}$ \\
\hline $2.63 \times 10^{-5}$ & $1.22 \times 10^{-3}$ & $3.12 \times 10^{1}$ \\
$2.63 \times 10^{-5}$ & $1.84 \times 10^{-3}$ & $4.77 \times 10^{1}$ \\
$2.63 \times 10^{-5}$ & $2.45 \times 10^{-3}$ & $6.18 \times 10^{1}$ \\
$2.63 \times 10^{-5}$ & $3.07 \times 10^{-3}$ & $7.91 \times 10^{1}$ \\
\hline \multicolumn{3}{c}{$k_{2}=2.56 \times 10^{4} \mathrm{M}^{-1} \mathrm{~s}^{-1}$} \\
\hline
\end{tabular}

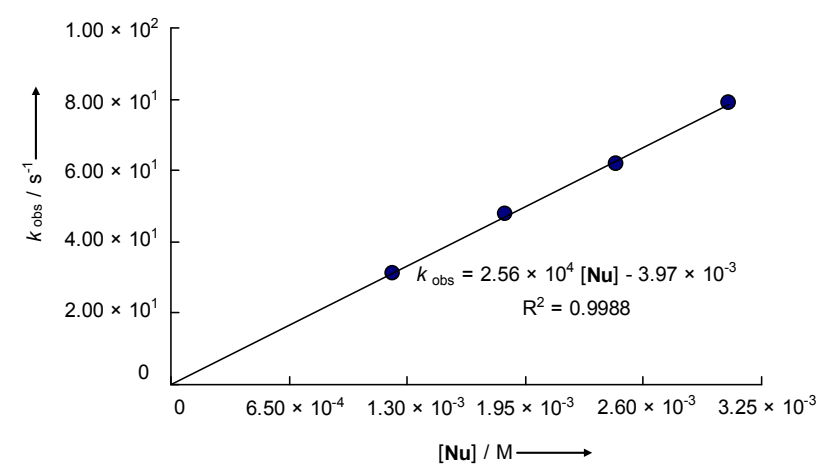

Kinetics of the reactions of potassium 6-methyl-2,4-dioxotetrahydro-2H-pyran-3-ide with carbocation $\mathbf{2 k}(\mathbf{2 k - B F})$ in $\mathrm{DMSO}$ at $20{ }^{\circ} \mathrm{C}$ (stopped-flow UV-Vis spectrometry, $\lambda=630$ $\mathrm{nm})$.

\begin{tabular}{ccc}
\hline$[\mathbf{2 k}]_{0} / \mathrm{M}$ & {$[\mathbf{1 4}] / \mathrm{M}$} & $k_{\mathrm{obs}} / \mathrm{s}^{-1}$ \\
\hline $4.72 \times 10^{-5}$ & $2.12 \times 10^{-3}$ & $1.90 \times 10^{1}$ \\
$4.72 \times 10^{-5}$ & $3.18 \times 10^{-3}$ & $2.88 \times 10^{1}$ \\
$4.72 \times 10^{-5}$ & $4.24 \times 10^{-3}$ & $3.72 \times 10^{1}$ \\
$4.72 \times 10^{-5}$ & $5.30 \times 10^{-3}$ & $4.47 \times 10^{1}$ \\
$4.72 \times 10^{-5}$ & $6.36 \times 10^{-3}$ & $5.20 \times 10^{1}$ \\
\hline \multicolumn{3}{c}{$k_{2}=7.73 \times 10^{3} \mathrm{M}^{-1} \mathrm{~s}^{-1}$} \\
\hline
\end{tabular}

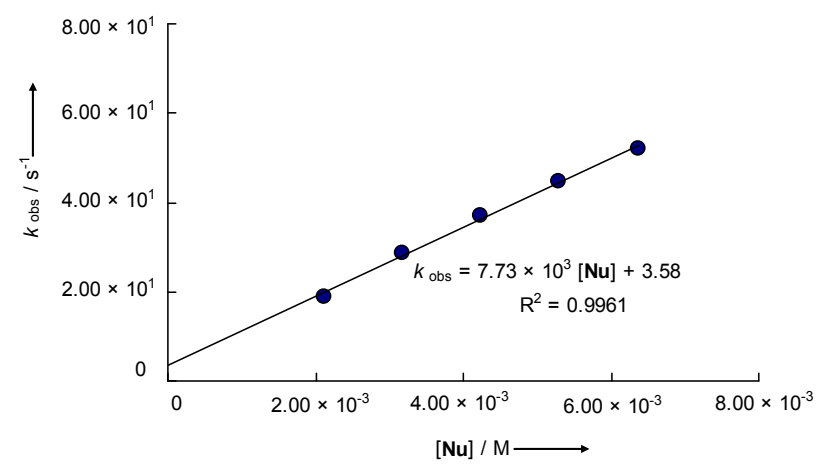




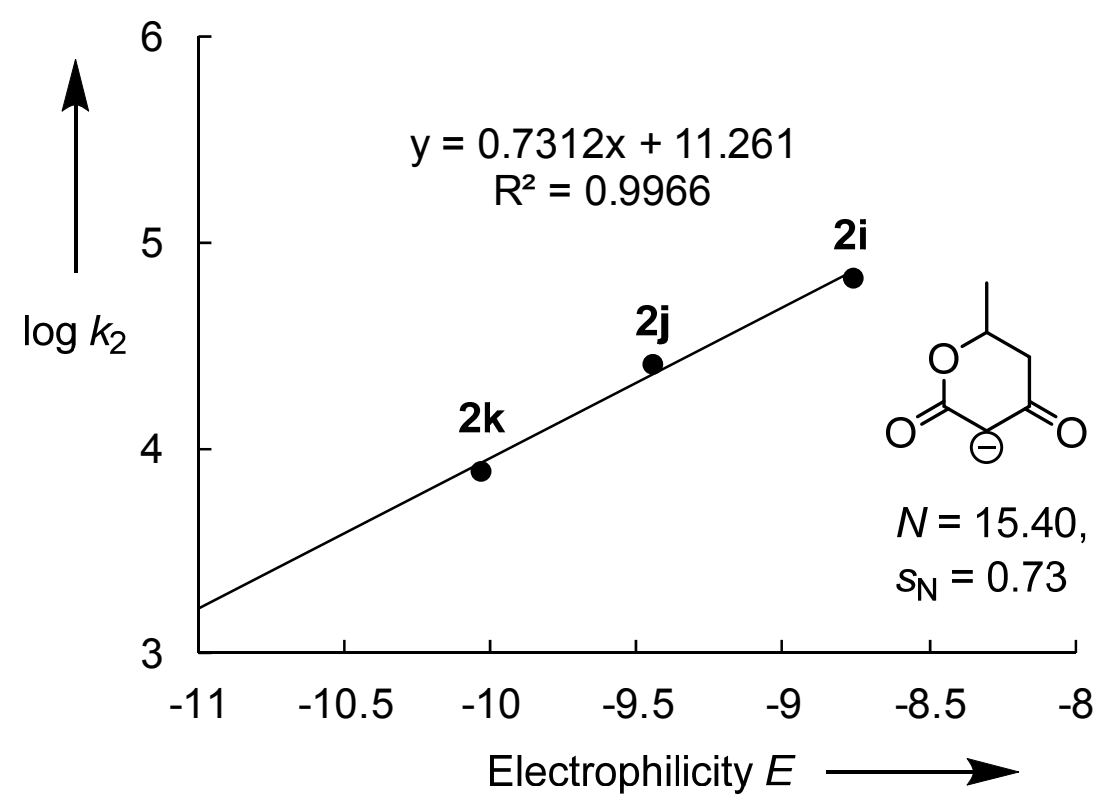

Figure S5. Determination of the nucleophilicity and sensitivity parameters $N$ and $s_{\mathrm{N}}$ of 6methyl-2,4-dioxotetrahydro-2H-pyran-3-ide (counter-ion: $\mathrm{K}^{+}$, in DMSO) 


\section{Crystallographic Data}

The data collections were performed on a Bruker D8Venture (MoK $\alpha$ radiation, $123 \mathrm{~K}$, 4) or an Oxford Diffraction XCalibur diffractometer (MoKa radiation, $173 \mathrm{~K}, \mathbf{8}$ and 9). The structures were solved by direct methods with SIR97 ${ }^{\mathrm{S} 13}$ and refined with SHELXL-97. 14

Supplementary crystallographic data for this paper have been deposited at The Cambridge Crystallographic Data Centre:

- CCDC 1474094 (4, rv305),

- CCDC 1445166 (8, po046), and

- $\operatorname{CCDC} 1445164$ (9, qo065).

These data can be obtained free of charge from The Cambridge Crystallographic Data Centre via www.ccdc.cam.ac.uk/data_request/cif. 
Crystallographic data for 4 (rv305)

net formula
$M_{\mathrm{r}} / \mathrm{g} \mathrm{mol}^{-1}$
crystal size/mm
crystal system
space group
$a / \AA$
$b / \AA$
$c / \AA$
$\alpha /{ }^{\circ}$
$\beta /{ }^{\circ}$
$\gamma /{ }^{\circ}$
$V / \AA^{3}$
$Z$

calc. density $/ \mathrm{g} \mathrm{cm}^{-3}$

$\mu / \mathrm{mm}^{-1}$

absorption correction

transmission factor range

refls. measured

$R_{\text {int }}$

mean $\sigma(I) / I$

$\theta$ range

observed refls.

$x, y$ (weighting scheme)

hydrogen refinement

refls in refinement

parameters

restraints

$R\left(F_{\text {obs }}\right)$

$R_{\mathrm{w}}\left(F^{2}\right)$

$S$

shift/error max $_{\text {max }}$

max electron density/e $\AA^{-3}$

min electron density/e $\AA^{-3}$
4

$\mathrm{C}_{23} \mathrm{H}_{25} \mathrm{ClO}_{4}$

400.895

$0.186 \times 0.048 \times 0.019$

monoclinic

$P 2{ }_{1} / n$

10.8984(11)

11.2830(13)

16.5940(18)

90

93.390(3)

90

2036.9(4)

4

1.3073(3)

0.214

multi-scan

0.8718-0.9579

15700

0.0819

0.0654

2.60-25.09

2450

$0.0435,0.3918$

constr

3607

257

0

0.0399

0.0995

1.015

0.001

0.229

$-0.304$ 


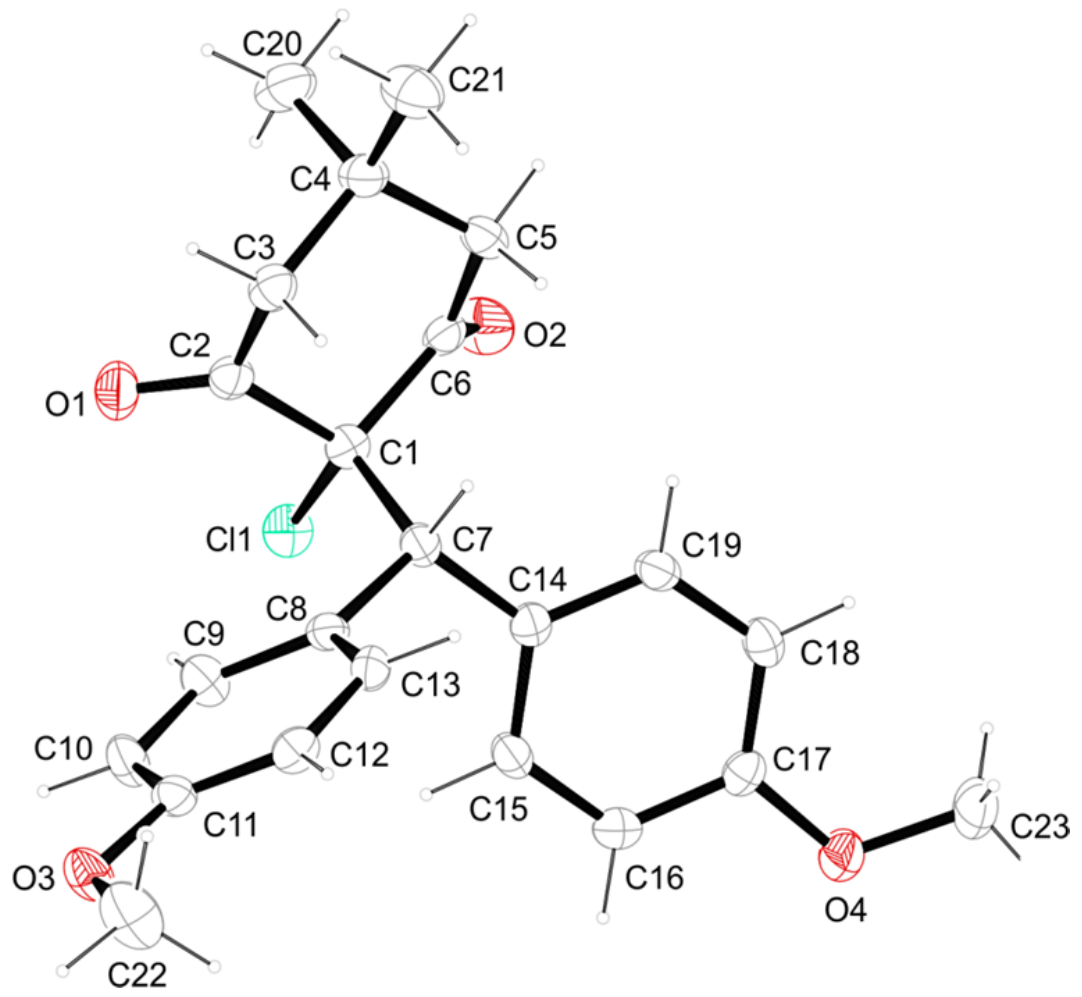

Figure S6. ORTEP drawing (25\% probability ellipsoids) of 2-(bis(4-methoxyphenyl)methyl)2-chloro-5,5-dimethylcyclohexane-1,3-dione (4). 
Crystallographic data for 8 (po046)

net formula

$M_{\mathrm{r}} / \mathrm{g} \mathrm{mol}^{-1}$

crystal size $/ \mathrm{mm}$

crystal system

space group

$a / \AA$

$b / \AA$

$c / \AA$

$\alpha /^{\circ}$

$\beta /{ }^{\circ}$

$\gamma /{ }^{\circ}$

$V / \AA^{3}$

$Z$

calc. density/g cm ${ }^{-3}$

$\mu / \mathrm{mm}^{-1}$

absorption correction

transmission factor range

refls. measured

$R_{\text {int }}$

mean $\sigma(I) / I$

$\theta$ range

observed refls.

$x, y$ (weighting scheme)

hydrogen refinement

refls in refinement

parameters

restraints

$R\left(F_{\text {obs }}\right)$

$R_{\mathrm{w}}\left(F^{2}\right)$

$S$

shift/error max $_{\text {max }}$

max electron density/e $\AA^{-3}$

min electron density/e $\AA^{-3}$
8

$\mathrm{C}_{35} \mathrm{H}_{42} \mathrm{~N}_{2} \mathrm{O}_{4}$

554.719

$0.27 \times 0.12 \times 0.09$

monoclinic

$P 2_{1} / n$

10.4286(6)

$17.5922(10)$

17.2786(11)

90

103.094(6)

90

3087.5(3)

4

$1.19339(12)$

0.077

'multi-scan'

0.93582-1.00000

22937

0.0640

0.1237

4.18-26.36

2910

0.0311, 0

constr

6259

378

0

0.0410

0.0795

0.763

0.001

0.193

$-0.136$ 


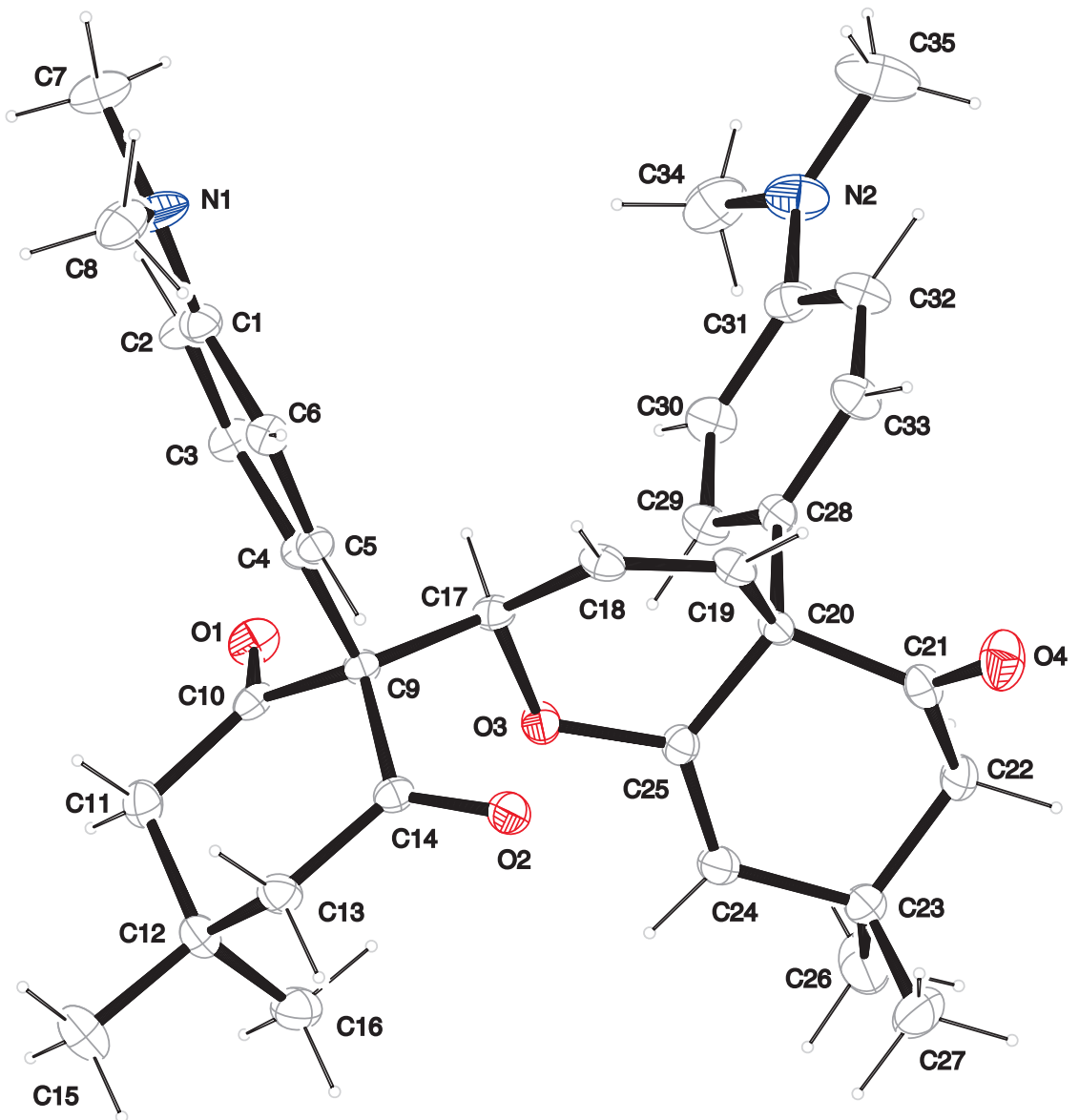

Figure S7. ORTEP drawing (25\% probability ellipsoids) of 2-(4-(dimethylamino)phenyl)-2(4a-(4-(dimethylamino)phenyl)-7,7-dimethyl-5-oxo-4a,5,6,7-tetrahydro-2H-chromen-2-yl)5,5-dimethylcyclohexane-1,3-dione (8). 
Crystallographic data for 9 (qo065)

net formula
$M_{\mathrm{r}} / \mathrm{g} \mathrm{mol}^{-1}$
crystal size/mm
crystal system
space group
$a / \AA$
$b / \AA$
$c / \AA$
$\alpha /{ }^{\circ}$
$\beta /{ }^{\circ}$
$\gamma /{ }^{\circ}$
$V / \AA^{3}$
$Z$

calc. density $/ \mathrm{g} \mathrm{cm}^{-3}$

$\mu / \mathrm{mm}^{-1}$

absorption correction

transmission factor range

refls. measured

$R_{\text {int }}$

mean $\sigma(I) / I$

$\theta$ range

observed refls.

$x, y$ (weighting scheme)

hydrogen refinement

refls in refinement

parameters

restraints

$R\left(F_{\text {obs }}\right)$

$R_{\mathrm{w}}\left(F^{2}\right)$

$S$

shift/error ${ }_{\max }$

max electron density/e $\AA^{-3}$

min electron density/e $\AA^{-3}$
9

$\mathrm{C}_{19} \mathrm{H}_{23} \mathrm{NO}_{3}$

313.391

$0.24 \times 0.18 \times 0.15$

triclinic

$P 1$ bar

9.7686(6)

$10.9965(7)$

$17.5770(11)$

74.913(6)

75.946(5)

$75.948(6)$

1735.66(19)

4

1.19933(13)

0.081

'multi-scan'

0.58268-1.00000

9569

0.0269

0.0535

4.13-25.35

4608

$0.0505,0.2460$

constr

6282

423

0

0.0499

0.1312

1.028

0.001

0.189

$-0.177$

There are two molecules in the asymmetric unit. 


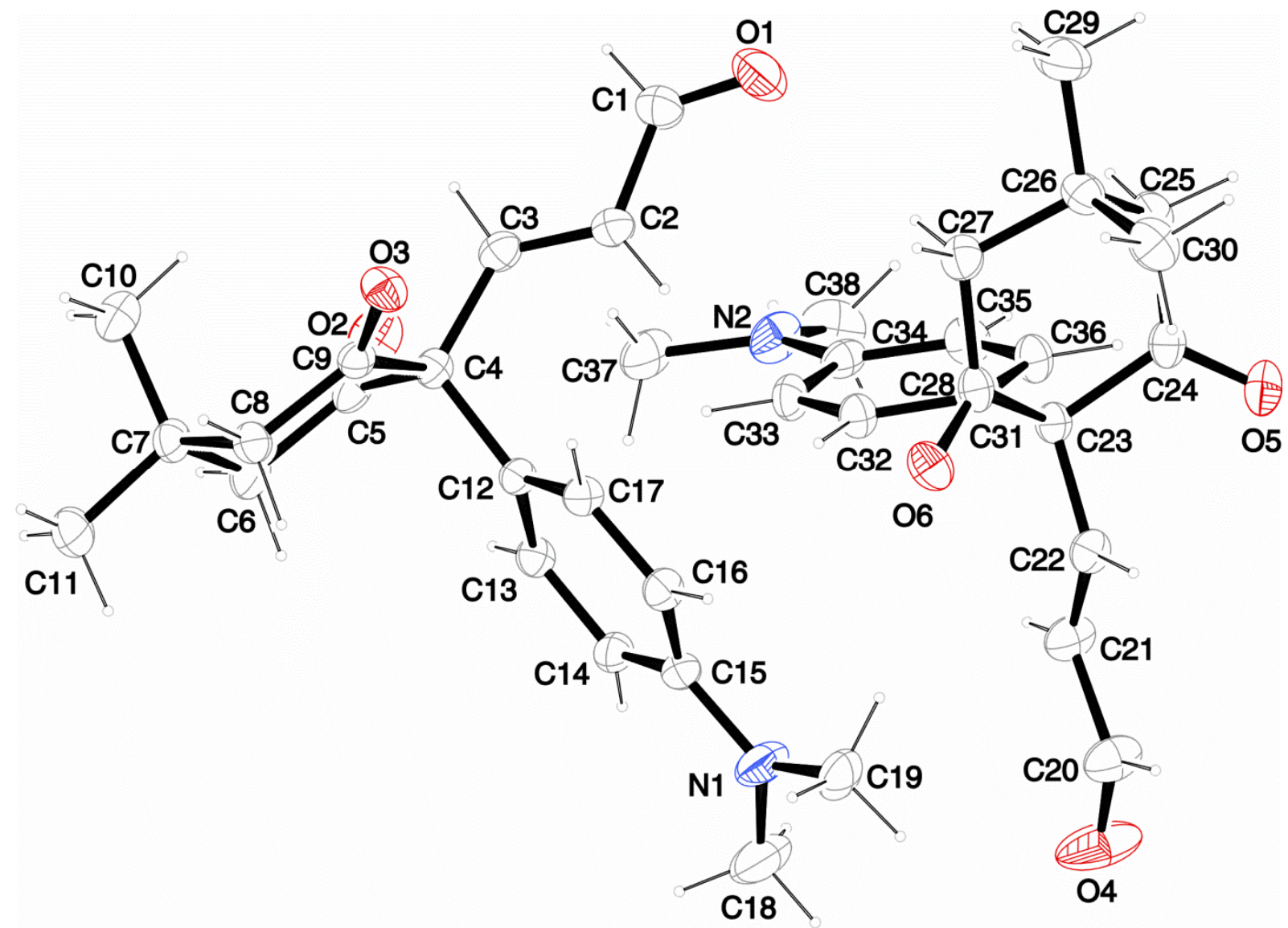

Figure S8. ORTEP drawing (25\% probability ellipsoids) of (E)-3-(1-(4-

(dimethylamino)phenyl)-4,4-dimethyl-2,6-dioxocyclohexyl)acrylaldehyde (9, both molecules of the asymmetric unit are shown). 


\section{Copies of the NMR spectra}
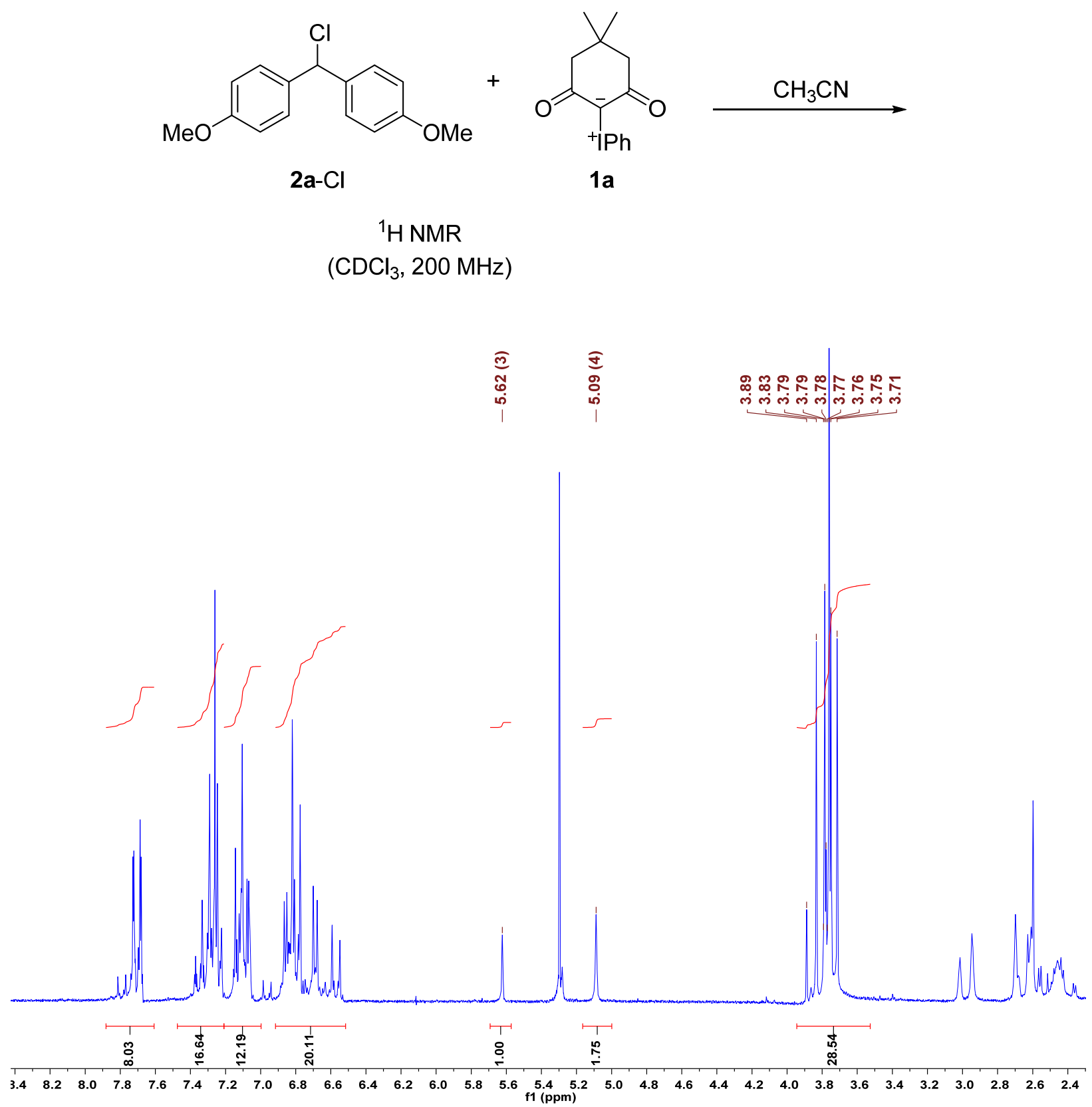

Figure S9. ${ }^{1} \mathrm{H}$ NMR spectrum of crude mixture obtained after reaction of $\mathbf{2 a}-\mathrm{Cl}$ and $\mathbf{1 a}$ in dichloromethane. - The NMR yields of $\mathbf{3}$ and $\mathbf{4}$ were calculated by division of the integral intensities at 5.62 and $5.09 \mathrm{ppm}$, respectively, through the gross integral intensity of the methoxy group resonances $(3.52-3.94 \mathrm{ppm}$ ) and multiplication of the results by 6 (two methoxy groups per one molecule of $\mathbf{2 a}-\mathrm{Cl}$ ) and $100 \%$. 


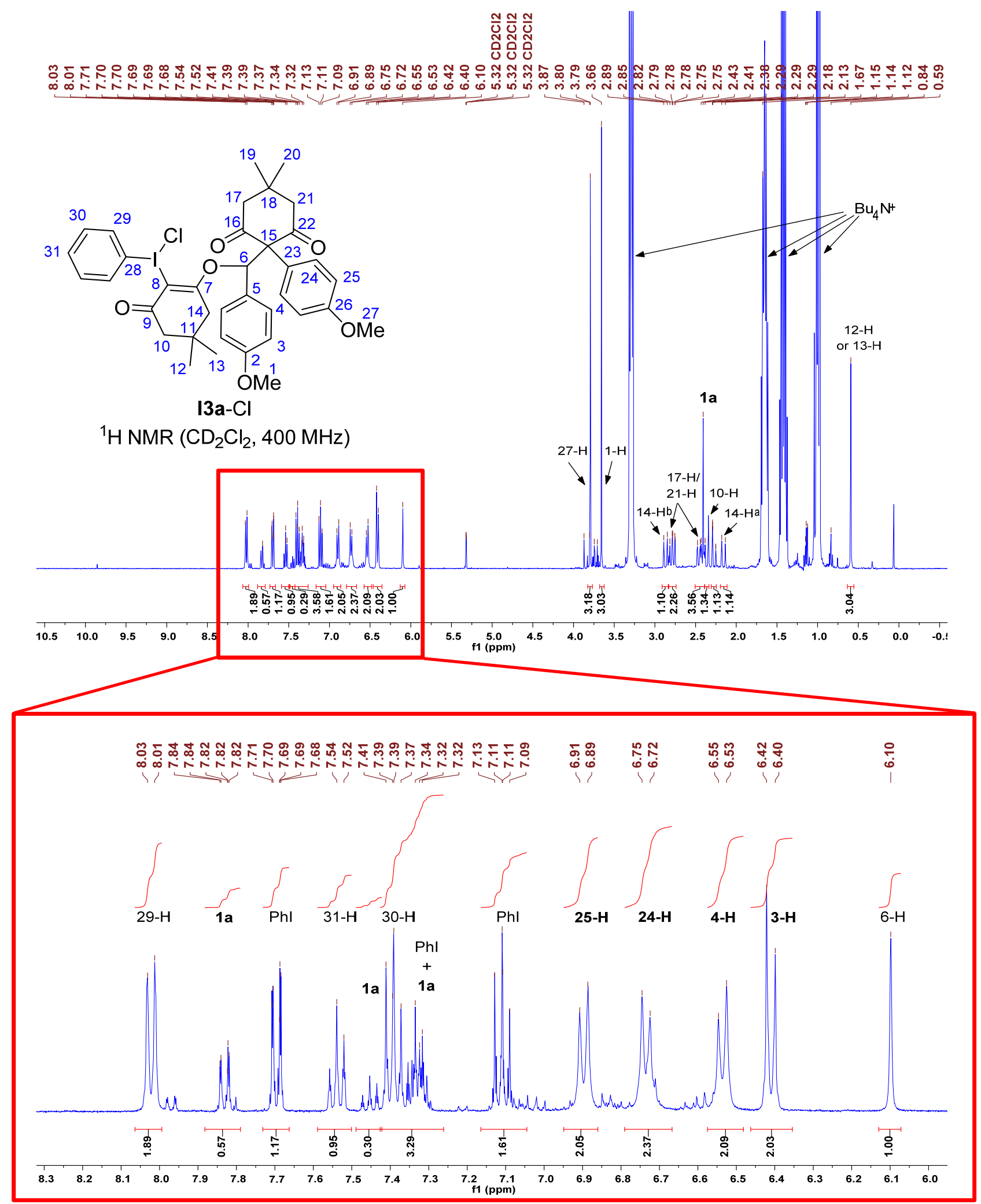

Figure S10. Assignment of resonances in the ${ }^{1} \mathrm{H}$ NMR spectrum acquired after mixing $\mathbf{2 a}$ $\mathrm{BF}_{4}$, $1 \mathrm{a}$ (2.06 equiv.), and $\mathrm{Bu}_{4} \mathrm{~N}^{+} \mathrm{Cl}^{-}$(1.1 equiv.) in $\mathrm{CD}_{2} \mathrm{Cl}_{2}$ (atom labels refer to the structure of $\mathbf{I 3 a}-\mathrm{Cl}$, further resonances were assigned to $\mathrm{Bu}_{4} \mathrm{~N}^{+}$, iodobenzene, and residual 1a). 


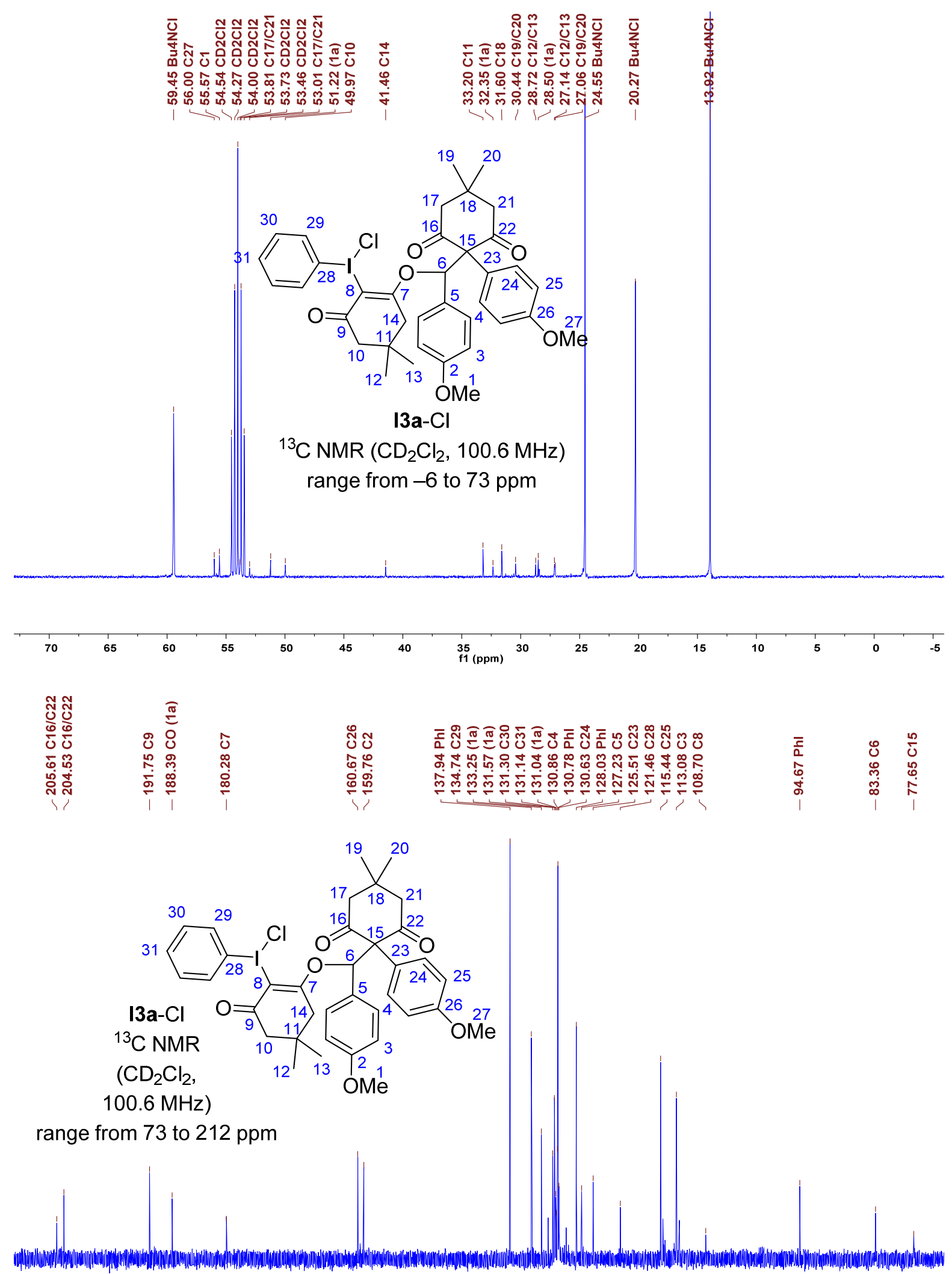

$\begin{array}{llllllllllllllllllllllllllllllll}210 & 205 & 200 & 195 & 190 & 185 & 180 & 175 & 170 & 165 & 160 & 155 & 150 & 145 & 140 & 135 & 130 & 125 & 120 & 115 & 110 & 105 & 100 & 95 & 90 & 85 & 80 & 75\end{array}$

Figure S11. Assignment of resonances in the ${ }^{13} \mathrm{C}$ NMR spectrum acquired after mixing 2a$\mathrm{BF}_{4}, \mathbf{1 a}\left(2.06\right.$ equiv.), and $\mathrm{Bu}_{4} \mathrm{~N}^{+} \mathrm{Cl}^{-}$(1.1 equiv.) in $\mathrm{CD}_{2} \mathrm{Cl}_{2}$ (atom labels refer to the structure of $\mathbf{I 3 a}-\mathrm{Cl}$, further resonances were assigned to $\mathrm{Bu}_{4} \mathrm{~N}^{+}$, iodobenzene, and residual 1a). 

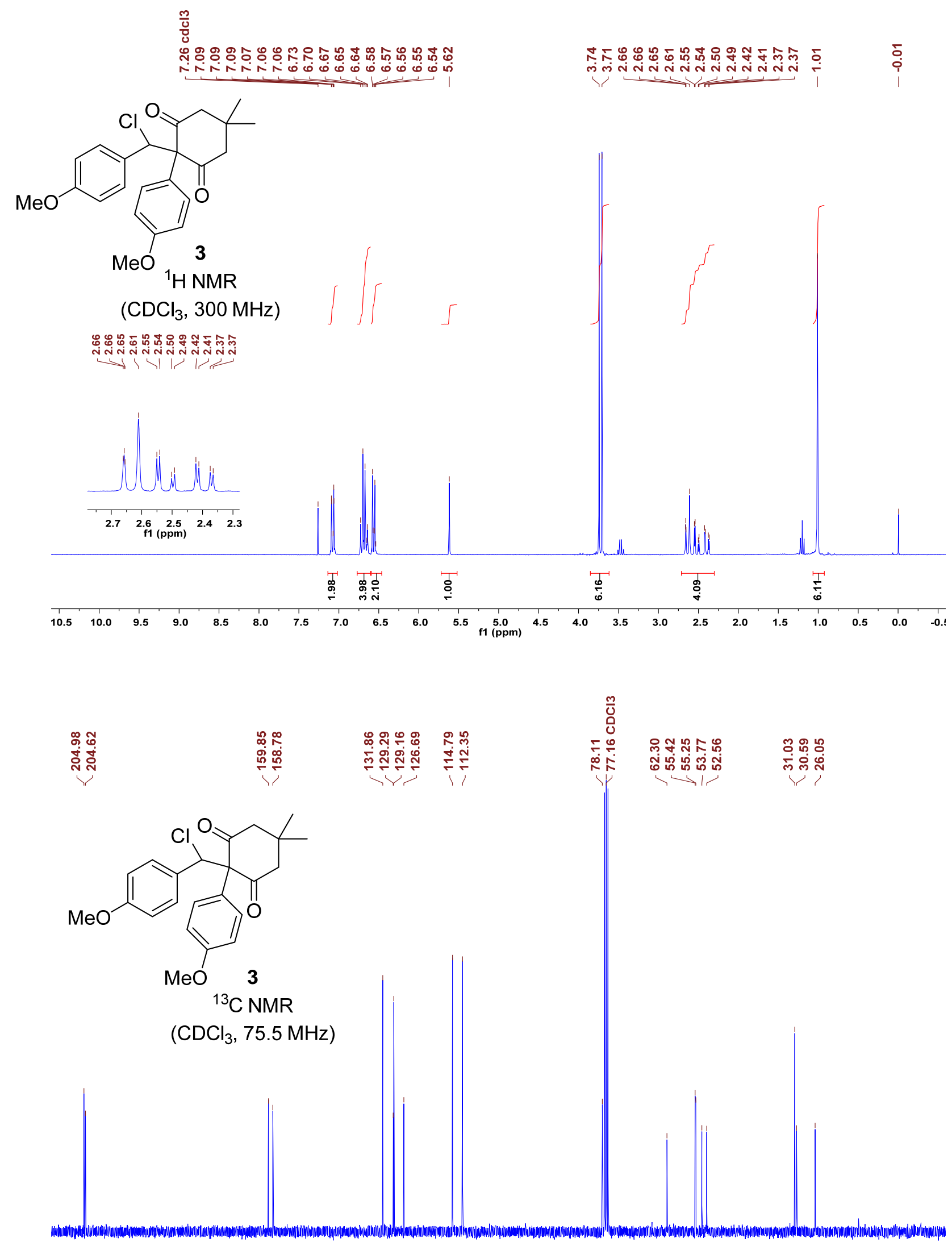

\begin{tabular}{llllllllllllllllllllll}
\hline 210 & 200 & 190 & 180 & 170 & 160 & 150 & 140 & 130 & 120 & $\underset{f 1}{110}\left(\begin{array}{c}100 \\
(\mathrm{ppm})\end{array}\right.$ & 90 & 80 & 70 & 60 & 50 & 40 & 30 & 20 & 10 & 0
\end{tabular} 


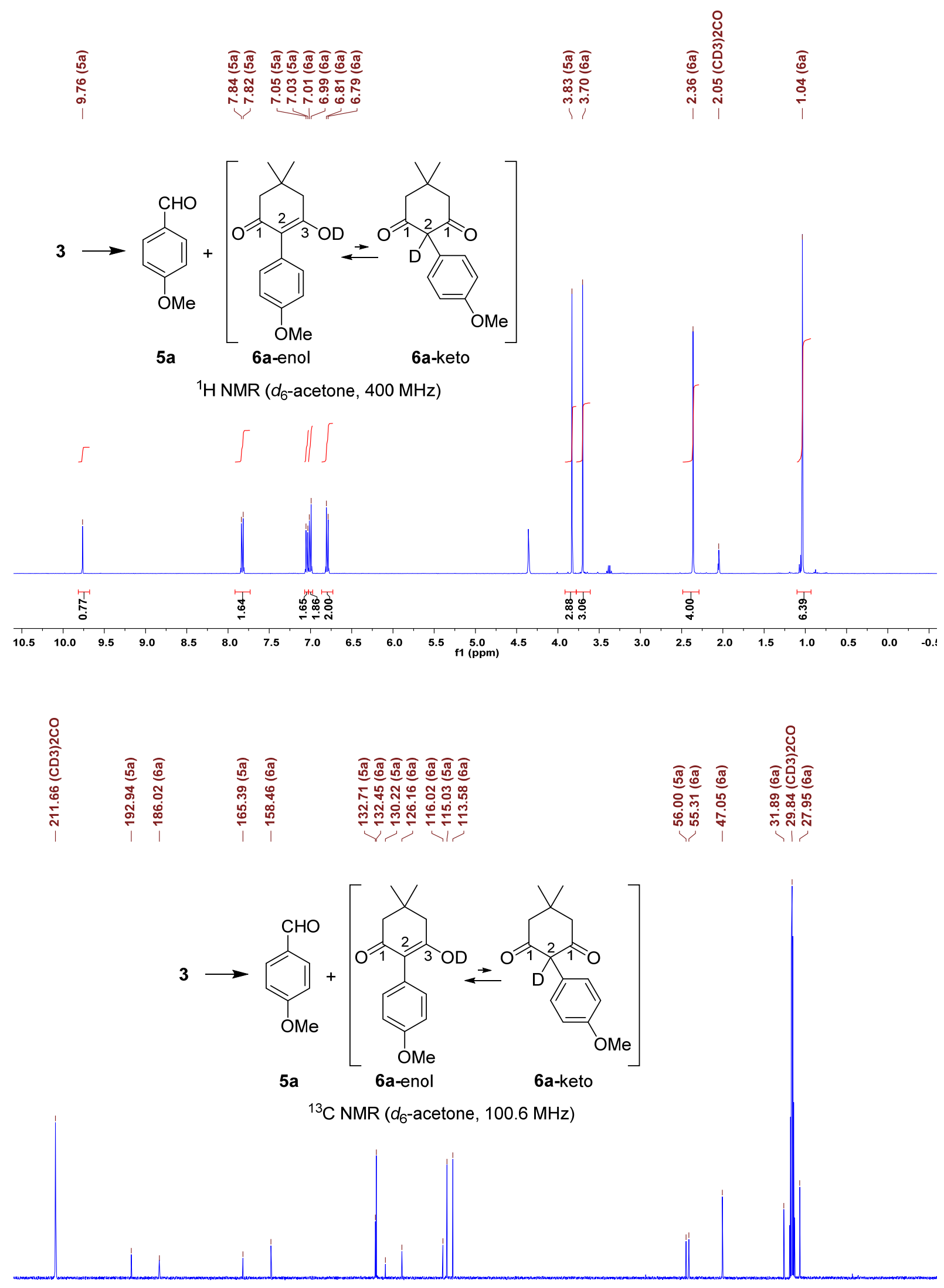

$\begin{array}{lllllllllllllllllllllllll}220 & 210 & 200 & 190 & 180 & 170 & 160 & 150 & 140 & 130 & 120 & 110 & 100 & 90 & 80 & 70 & 60 & 50 & 40 & 30 & 20 & 10 & 0\end{array}$ 

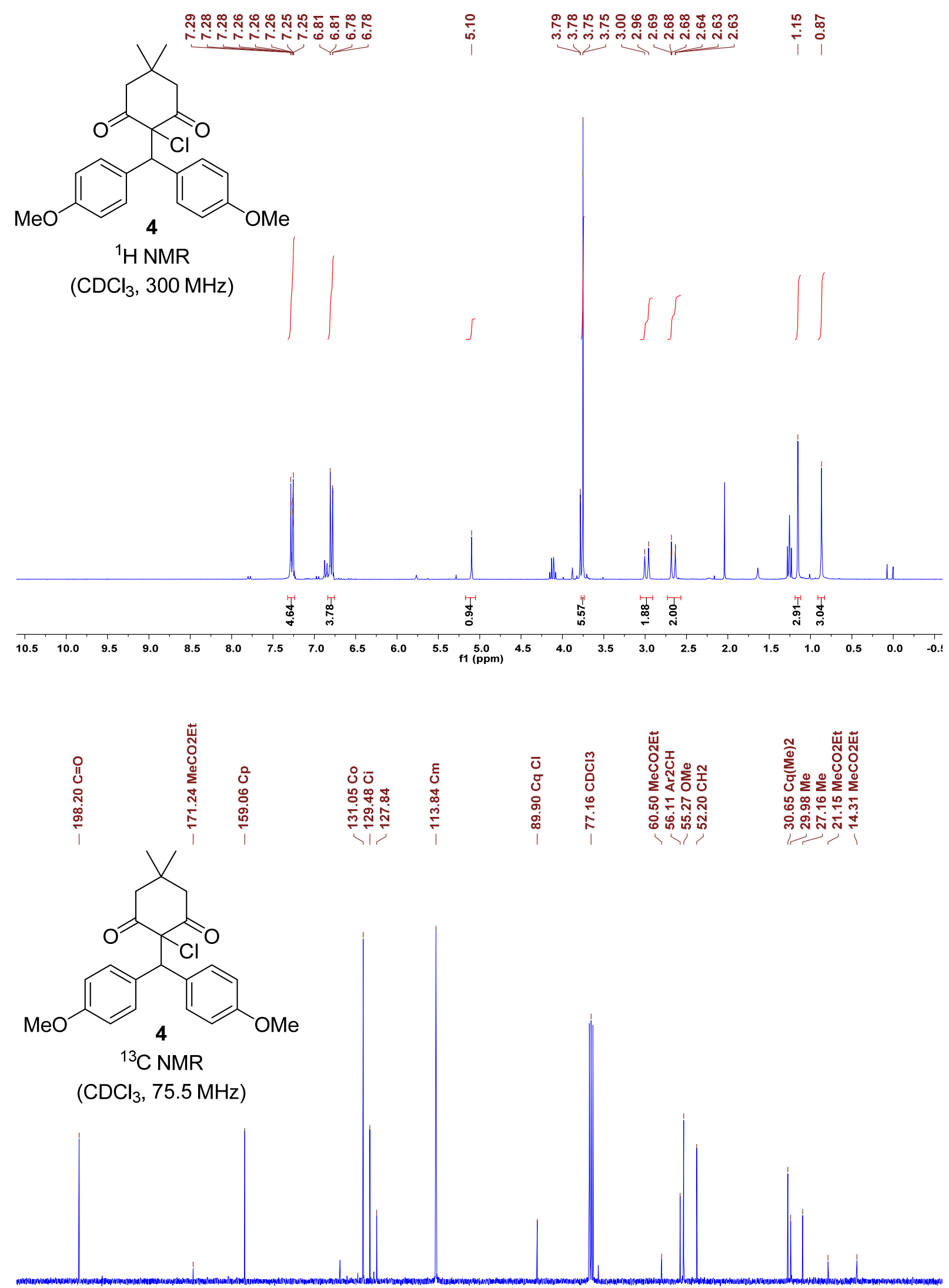

$\begin{array}{lllllllllllllllllllllll}210 & 200 & 190 & 180 & 170 & 160 & 150 & 140 & 130 & 120 & 110 & 100 & 90 & 80 & 70 & 60 & 50 & 40 & 30 & 20 & 10 & 0\end{array}$ 

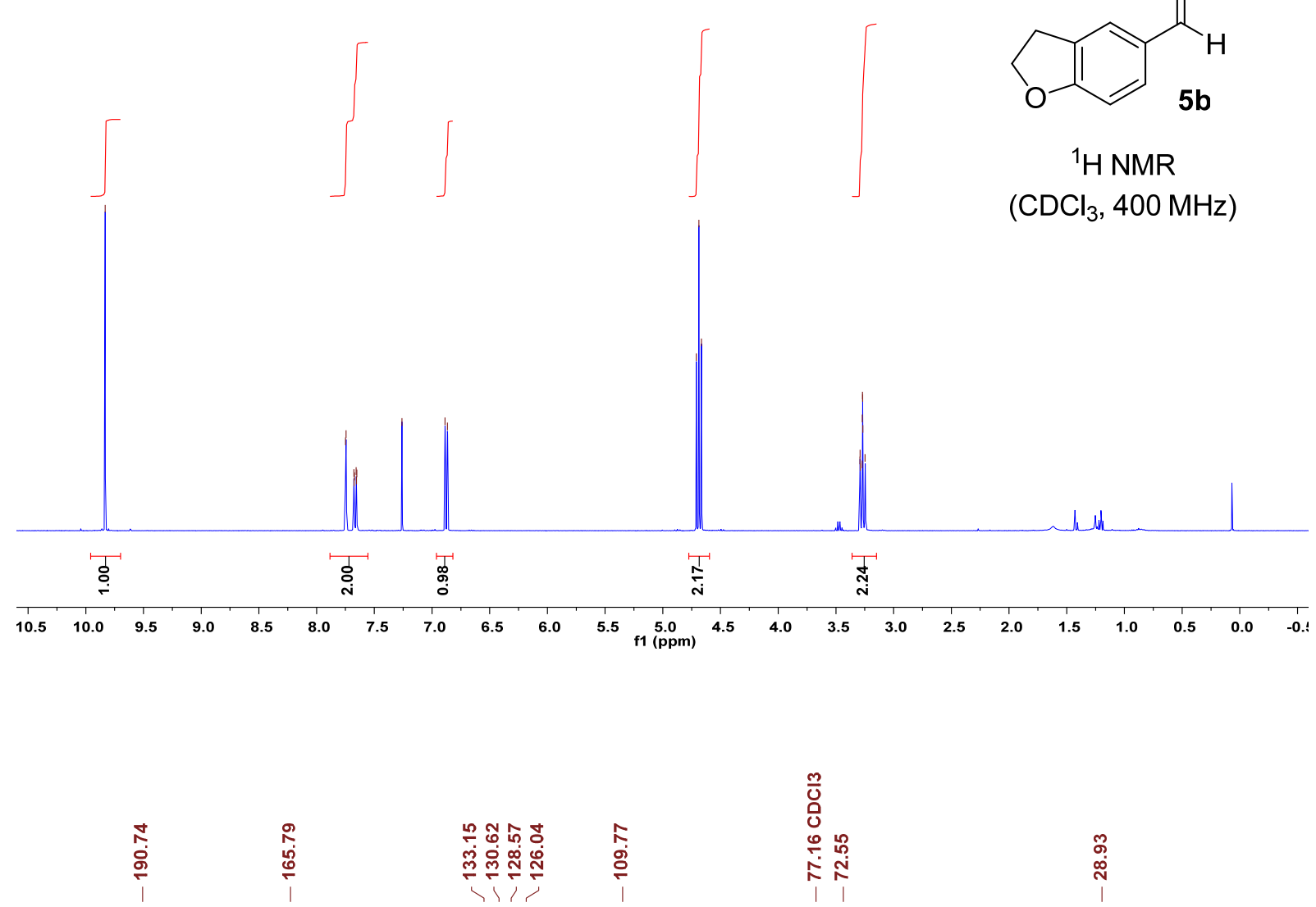<smiles>O=C(I)c1ccc2c(c1)CCO2</smiles>

${ }^{13} \mathrm{C}$ NMR

$\left(\mathrm{CDCl}_{3}, 100.6 \mathrm{MHz}\right)$

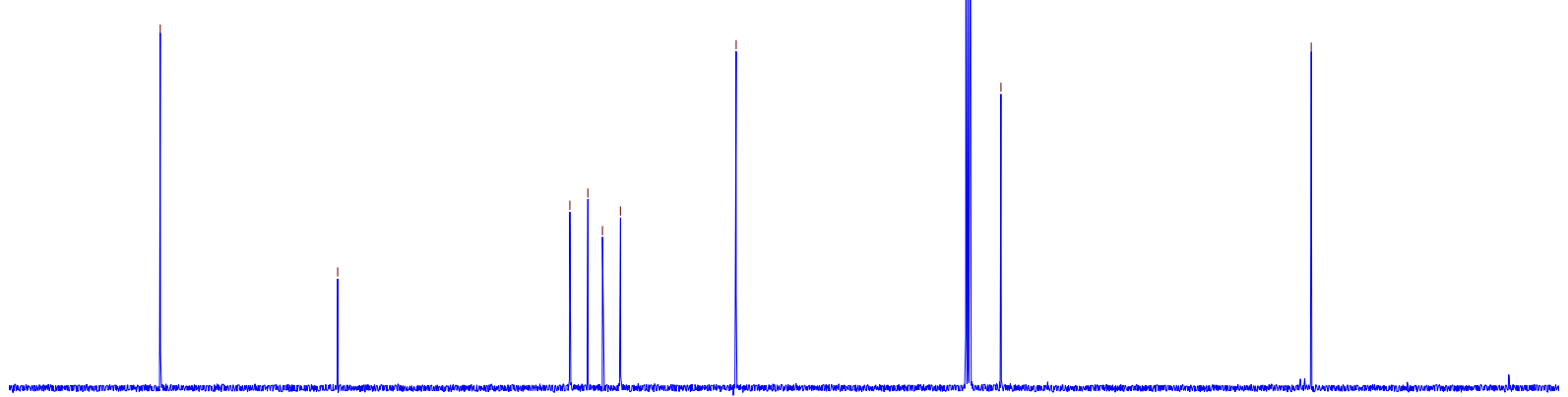

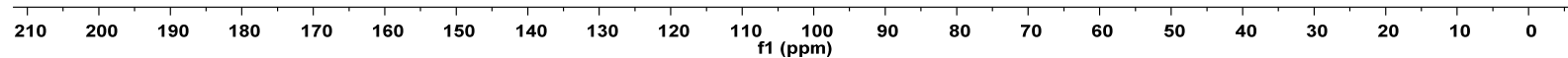



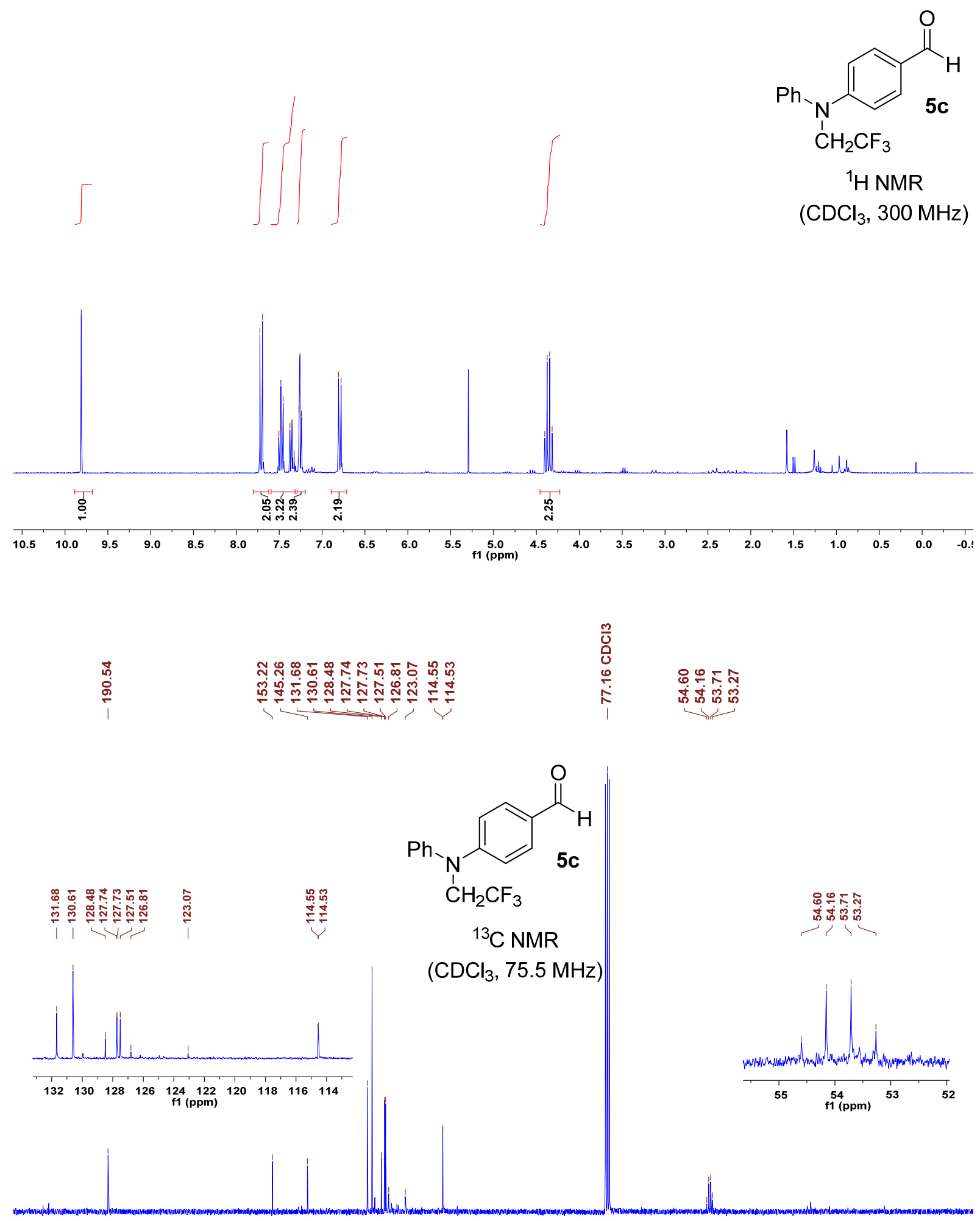

\begin{tabular}{lllllllllll}
\hline 210 & 200 & 190 & 180 & 170 & 160 & 150 & 140 & 130 & 120 & $\begin{array}{l}110 \\
\mathrm{f} 1(\mathrm{ppm})\end{array}$
\end{tabular} 


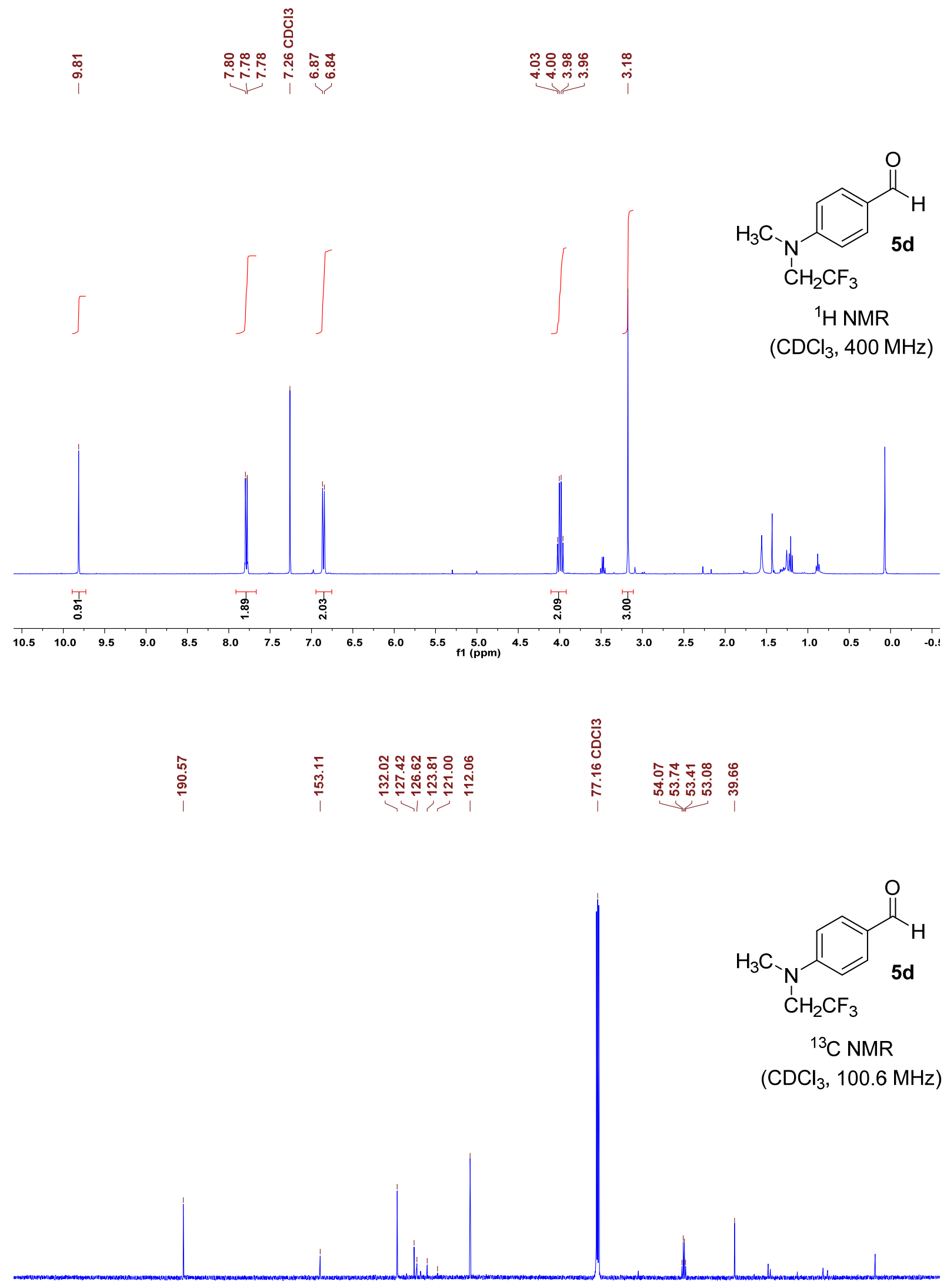

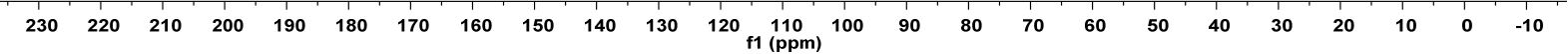




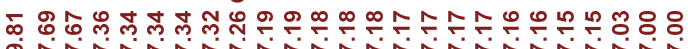

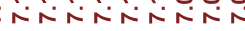

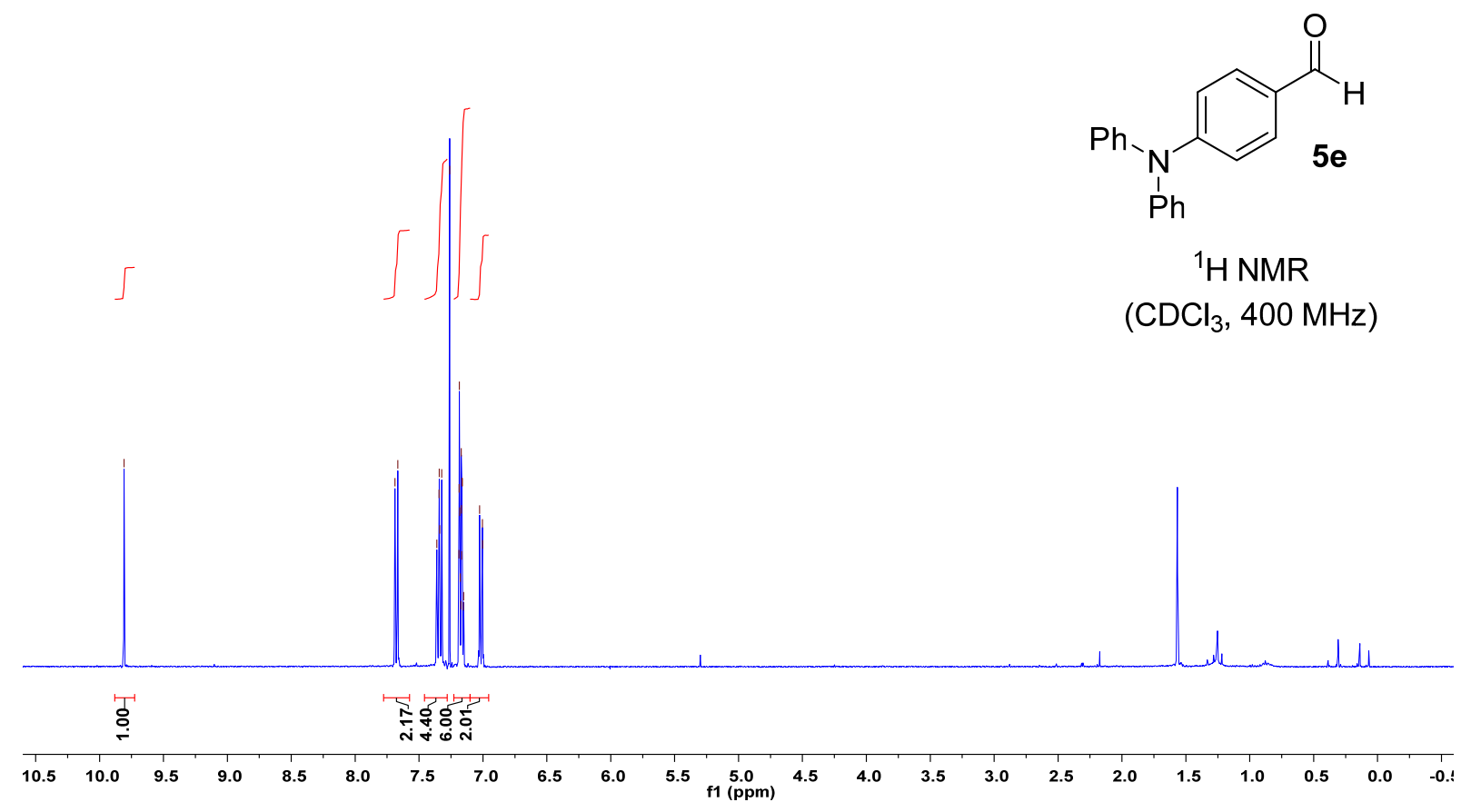

${ }^{1} \mathrm{H}$ NMR

$\mathrm{CDCl}_{3}, 400 \mathrm{MHz}$

ll

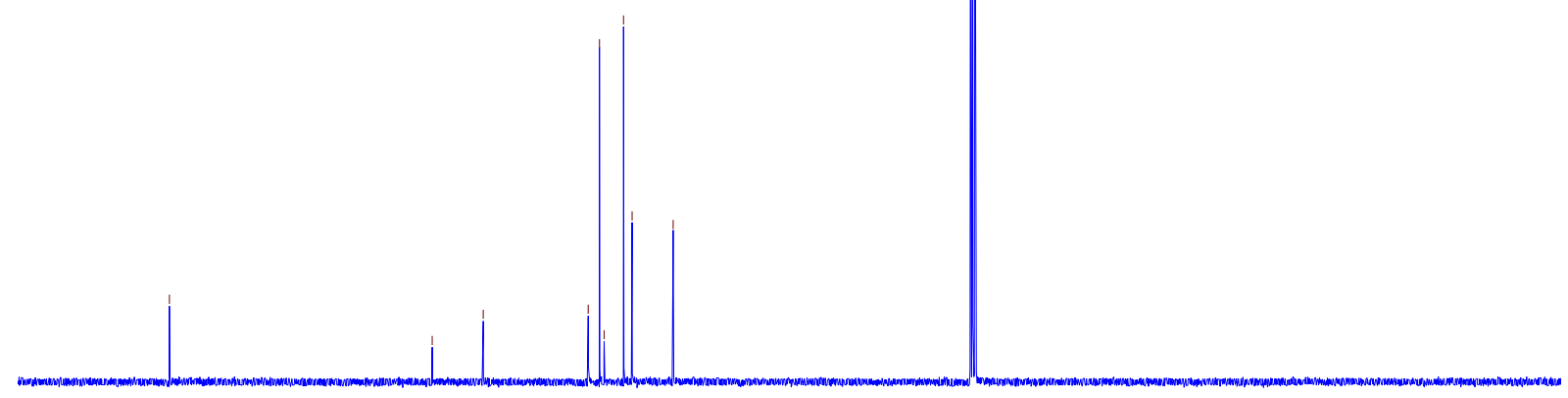

${ }^{13} \mathrm{C}$ NMR

$\left(\mathrm{CDCl}_{3}, 100.6 \mathrm{MHz}\right)$

$\begin{array}{llllllllllllllllllllll}210 & 200 & 190 & 180 & 170 & 160 & 150 & 140 & 130 & 120 & 110 & 100 & 90 & 80 & 70 & 60 & 50 & 40 & 30 & 20 & 10 & 0\end{array}$ 


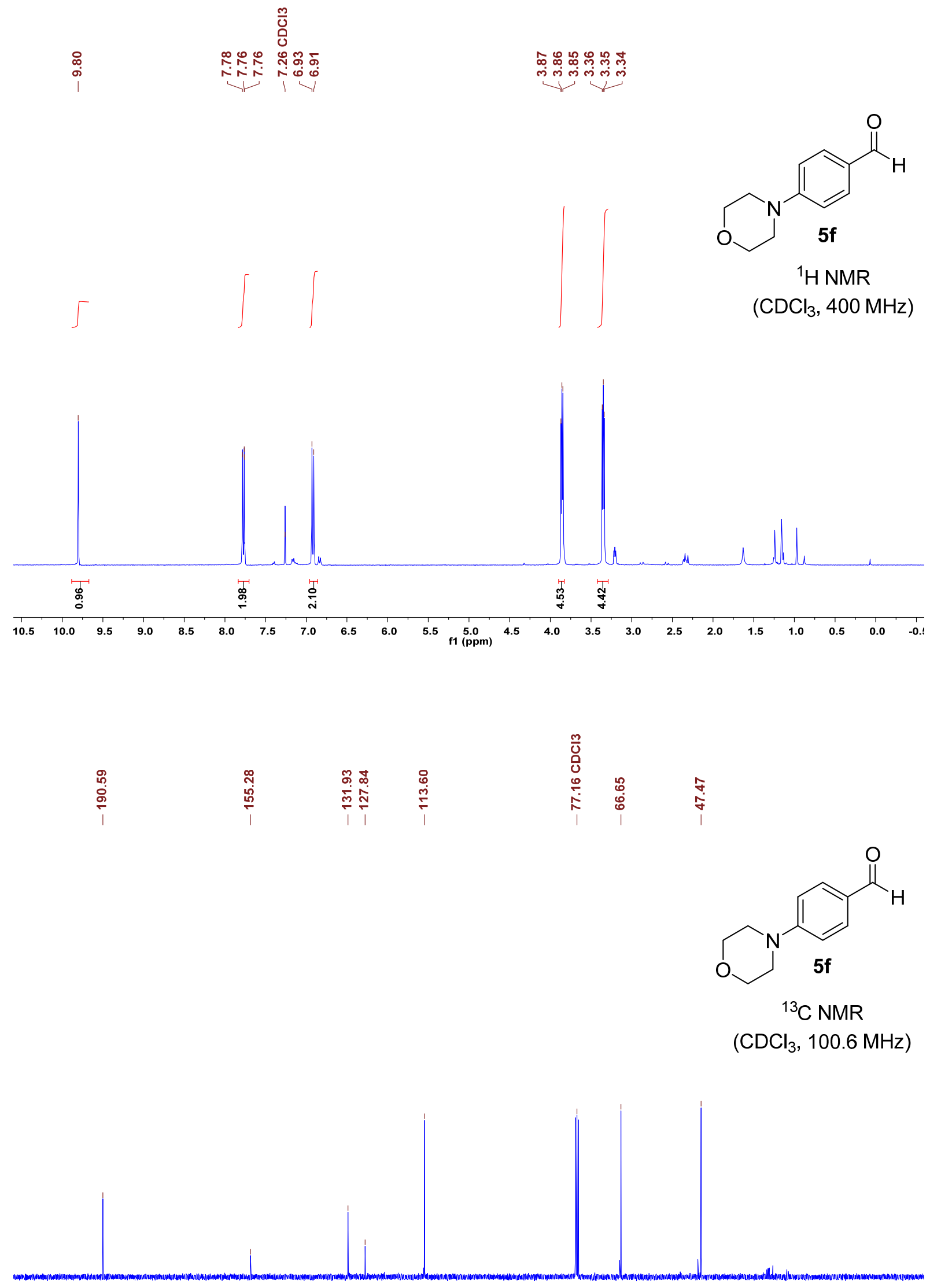

\begin{tabular}{lllllllllllllllllllllllll}
\hline 210 & 200 & 190 & 180 & 170 & 160 & 150 & 140 & 130 & 120 & 110 & 100 & 90 & 80 & 70 & 60 & 50 & 40 & 30 & 20 & 10 & 0
\end{tabular} 

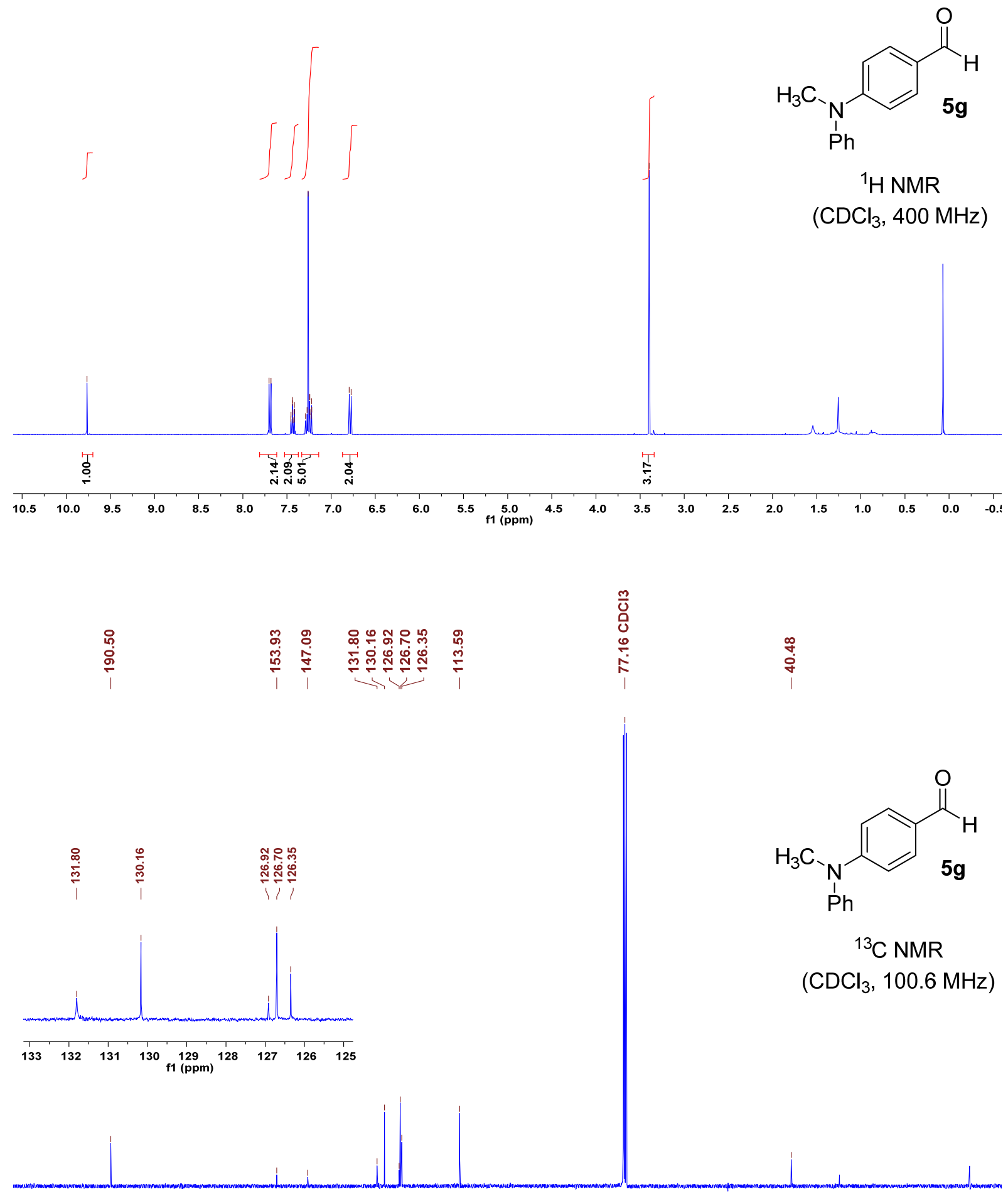

$\begin{array}{lllllllllll}210 & 200 & 190 & 180 & 170 & 160 & 150 & 140 & 130 & 120 & 110 \begin{array}{l}100 \\ \mathrm{f} 1(\mathrm{ppm})\end{array}\end{array}$ 

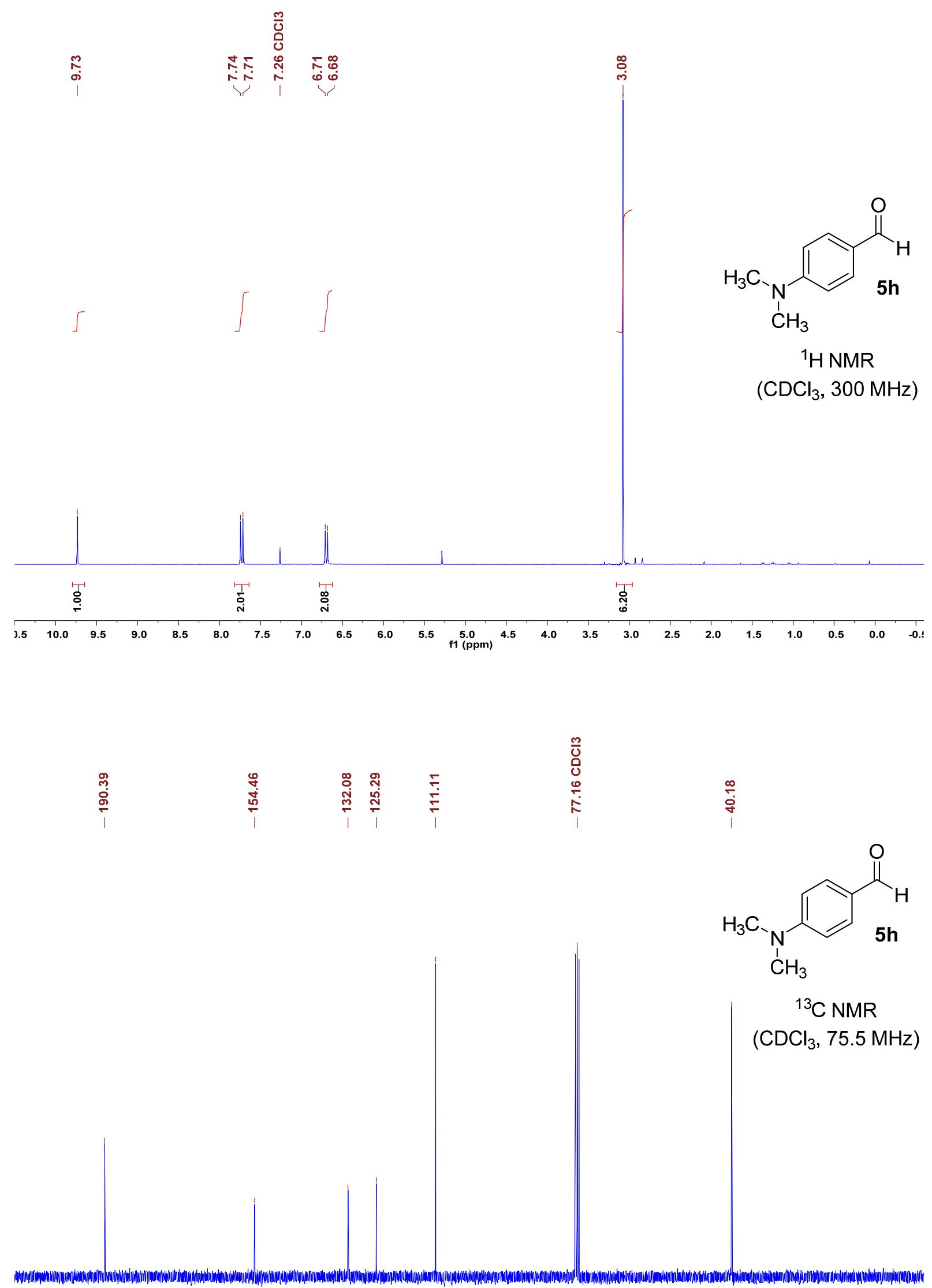

\begin{tabular}{llllllllllllllllllllllll}
\hline 210 & 200 & 190 & 180 & 170 & 160 & 150 & 140 & 130 & 120 & 110 & 100 & 90 & 80 & 70 & 60 & 50 & 40 & 30 & 20 & 10 & 0
\end{tabular} 
䓈

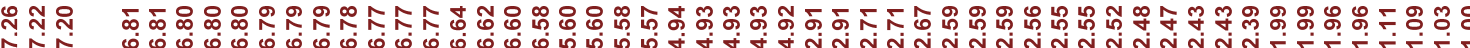

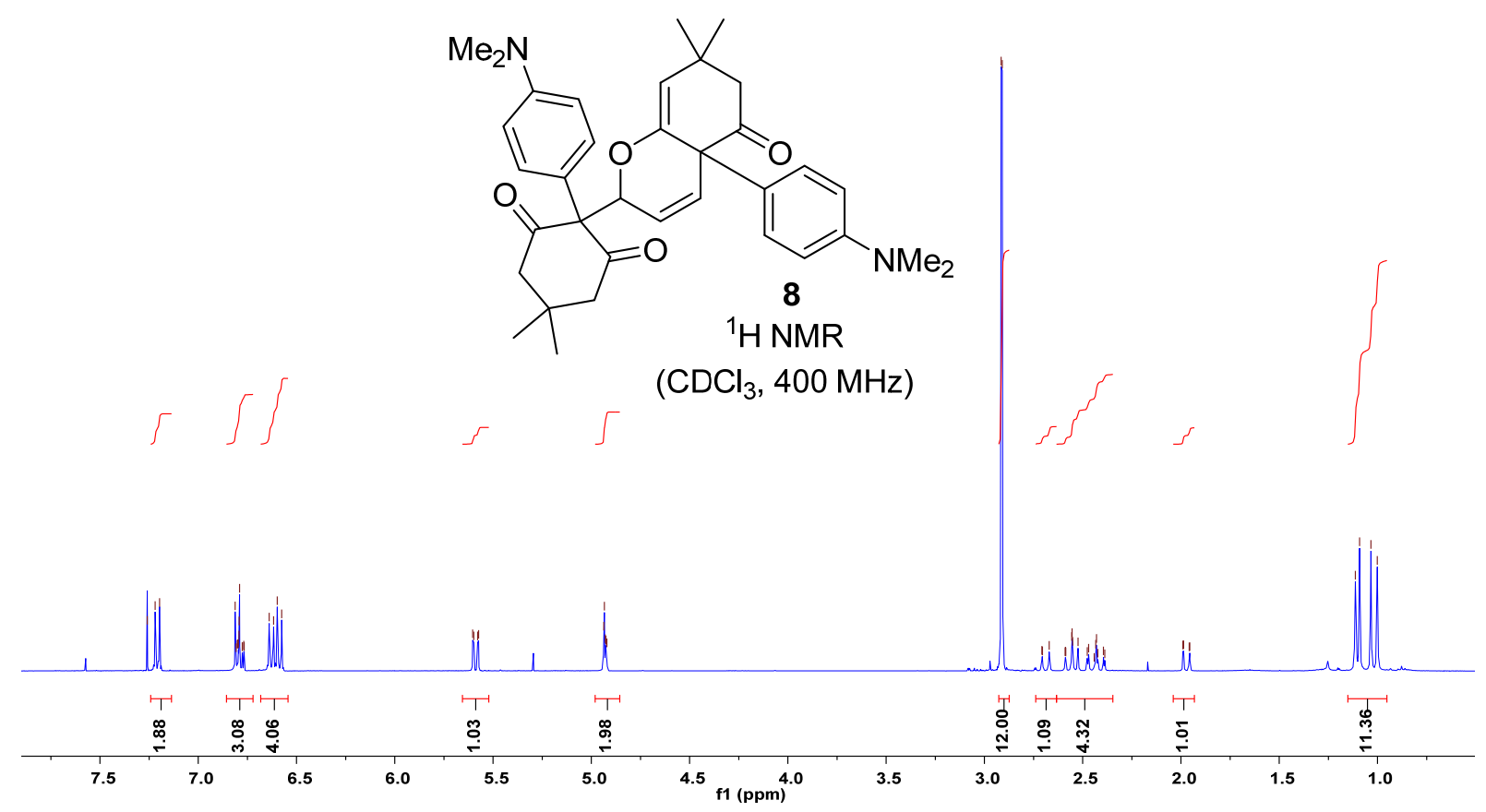

ถั่

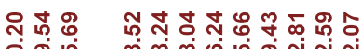

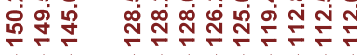

రับ

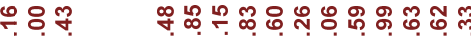

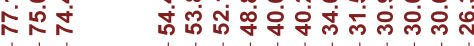

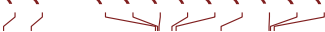
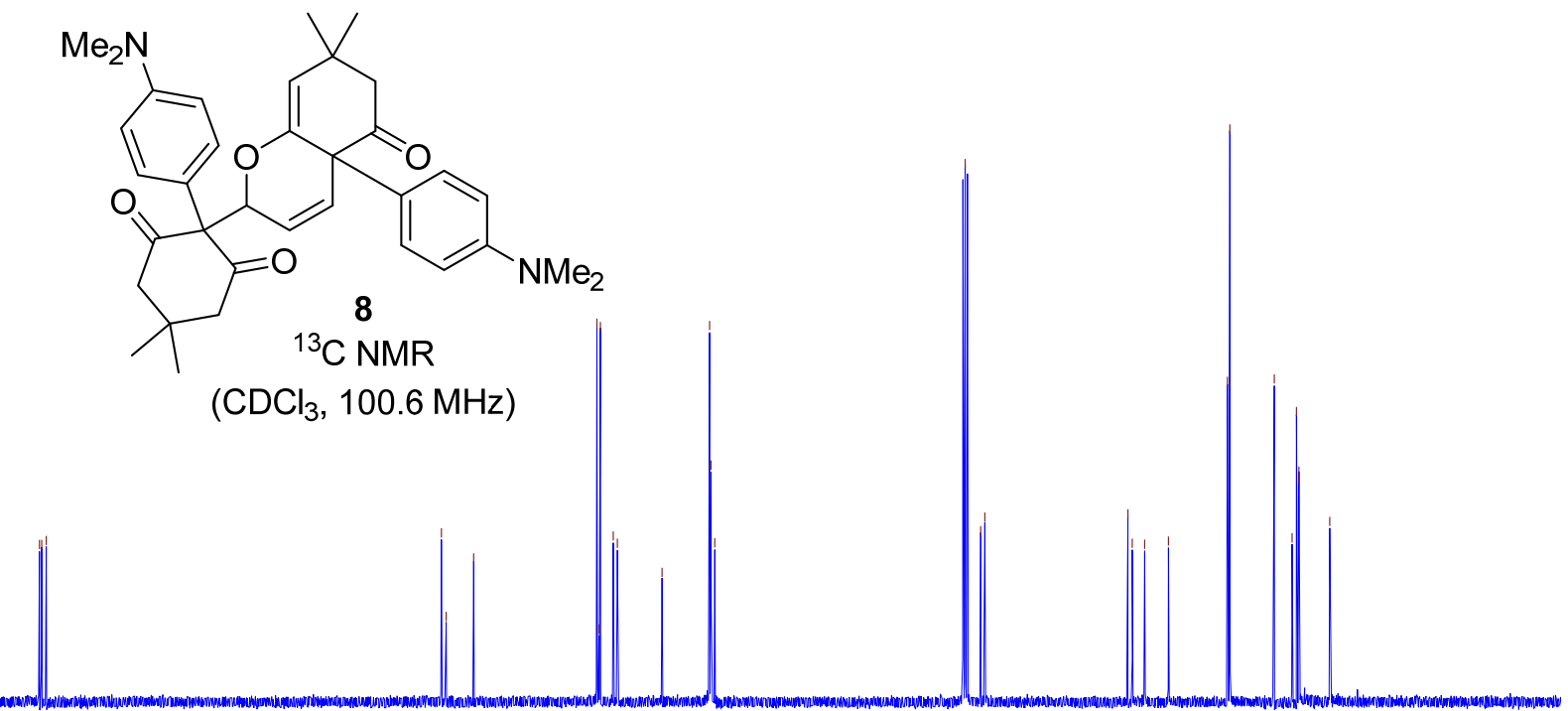

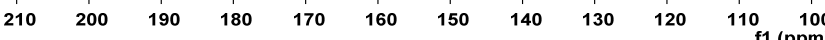




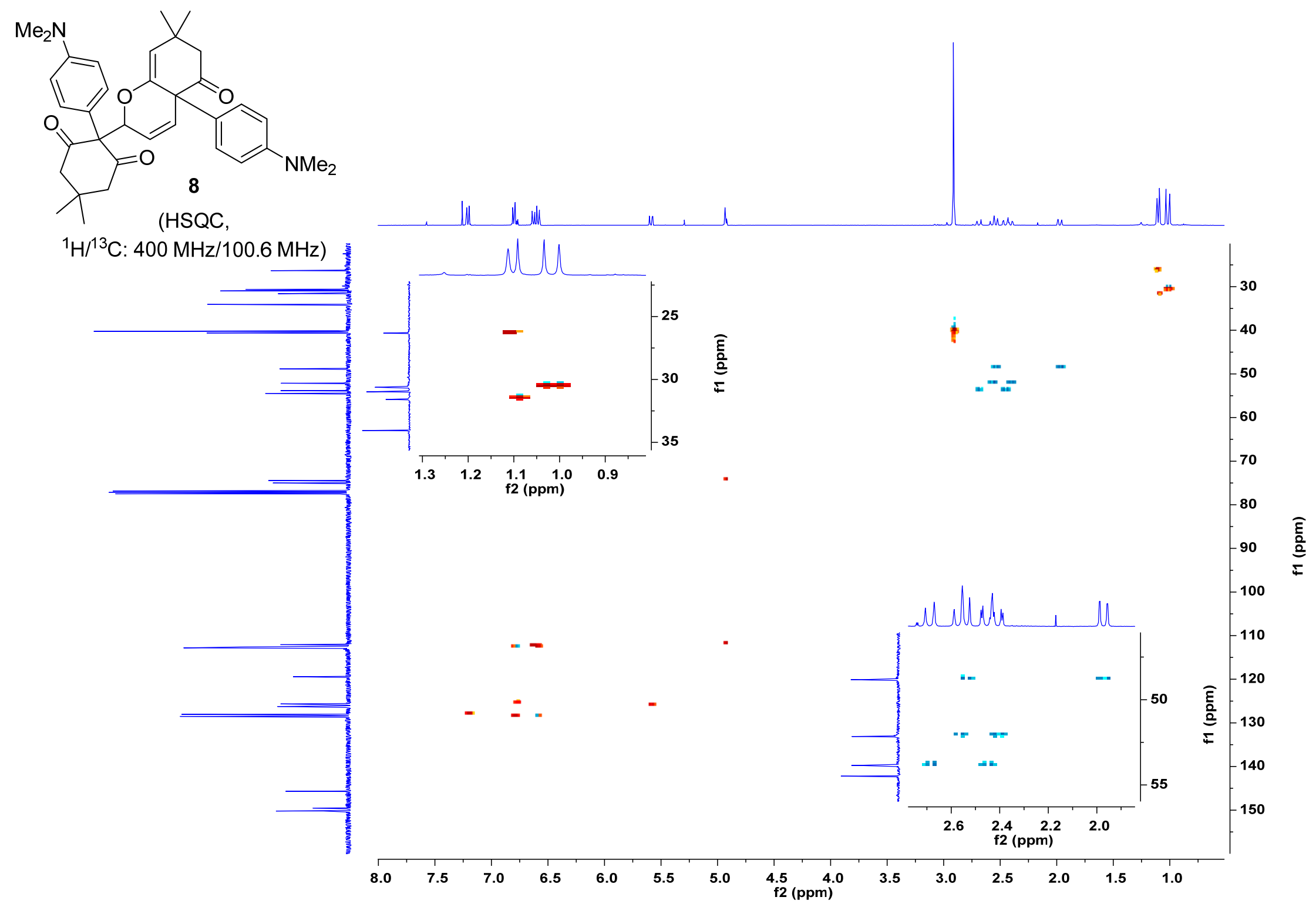



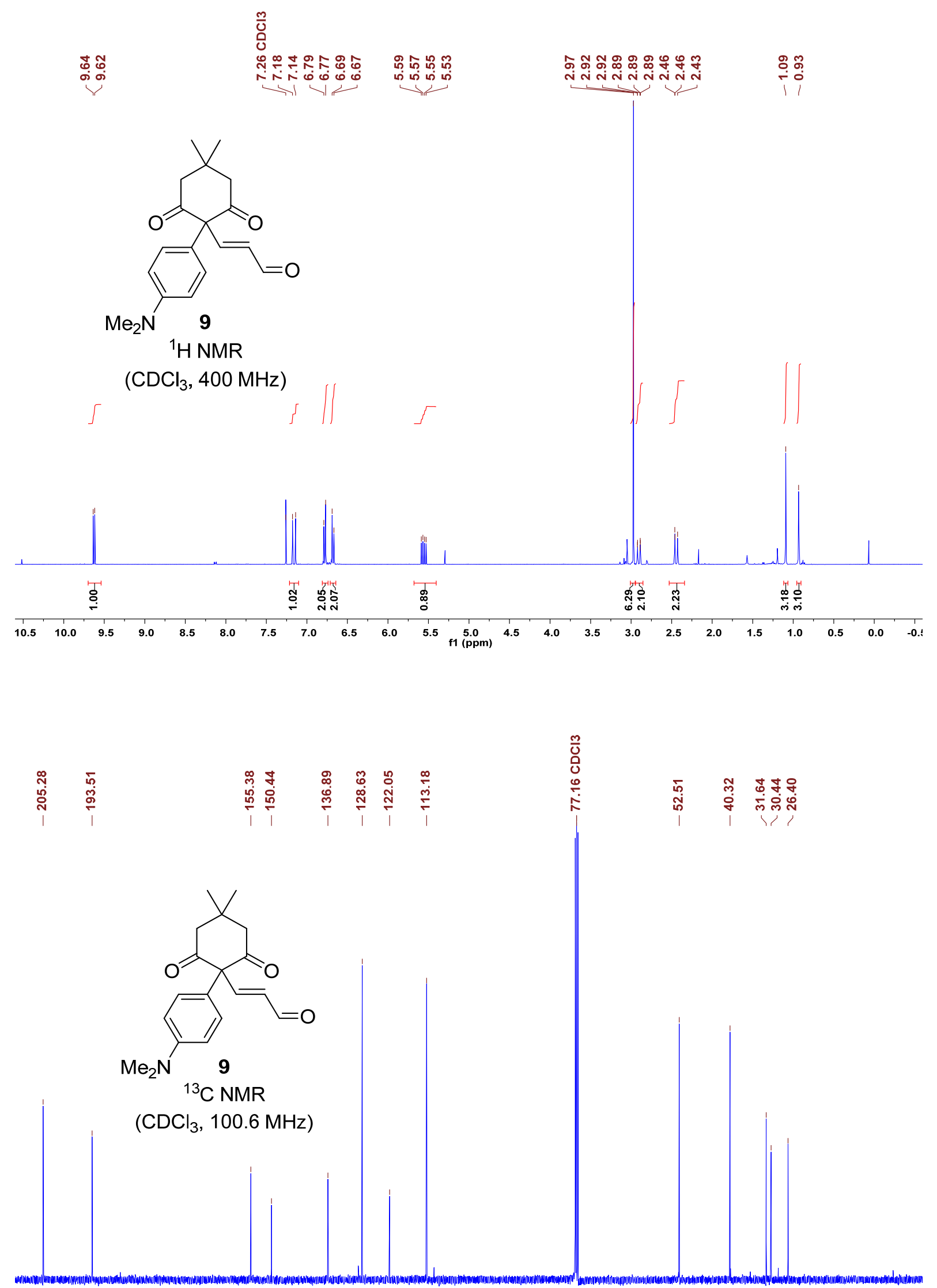

\begin{tabular}{llllllllllllllllllllllll}
\hline 210 & 200 & 190 & 180 & 170 & 160 & 150 & 140 & 130 & 120 & 110 & 100 & 90 & 80 & 70 & 60 & 50 & 40 & 30 & 20 & 10 & 0
\end{tabular} 
<smiles>CC1(C)CC(=O)C2(C(=O)C1)[C@H](c1ccccc1)[C@H]2C=O</smiles>

12

${ }^{1} \mathrm{H}$ NMR

s.

$\left(\mathrm{CDCl}_{3}, 400 \mathrm{MHz}\right)$

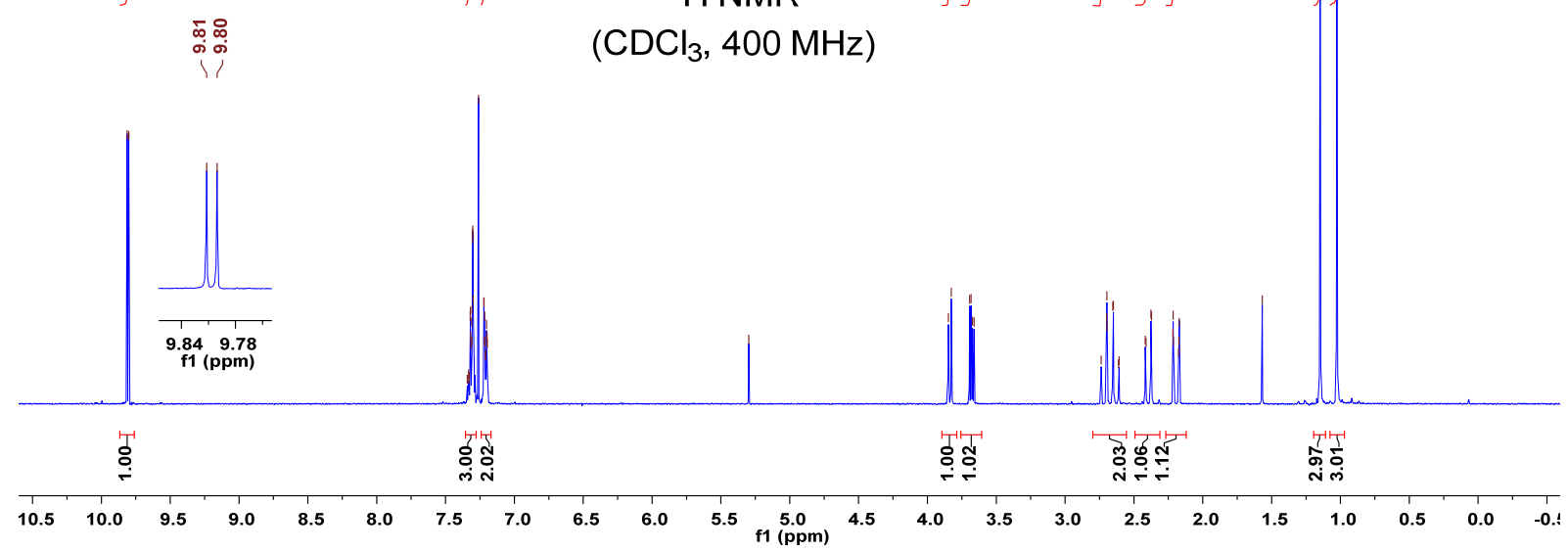

ชิธ

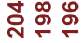

年金

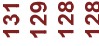

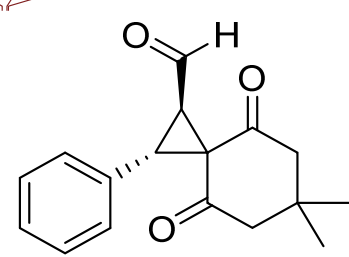

12

${ }^{13} \mathrm{C}$ NMR

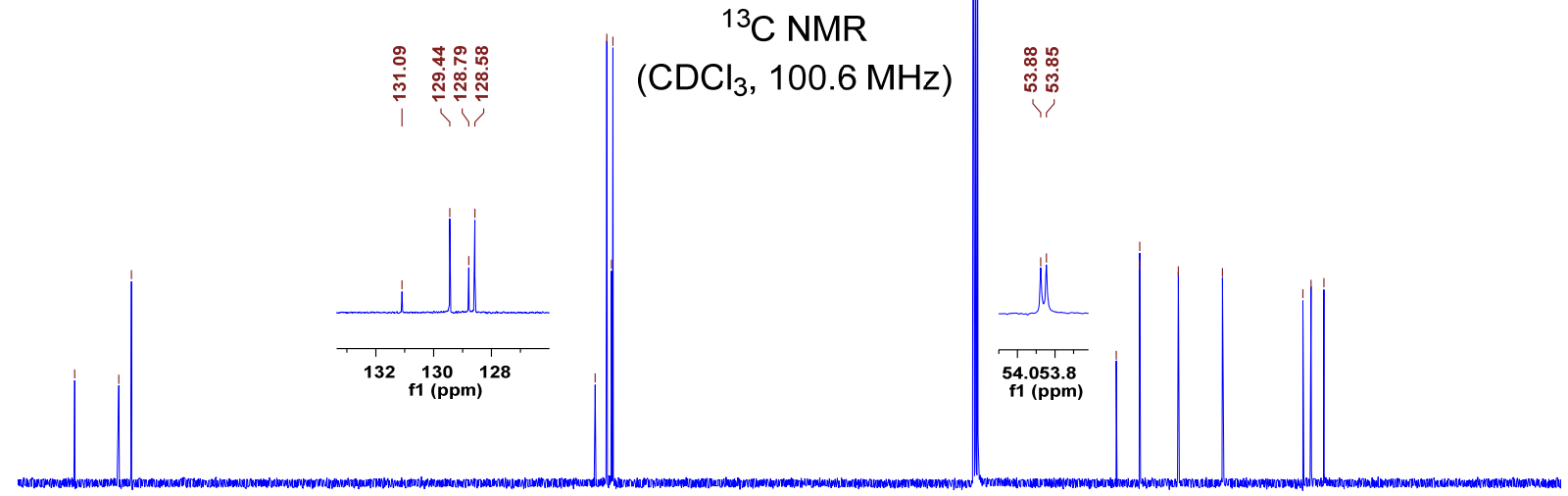

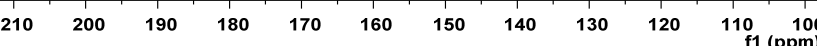

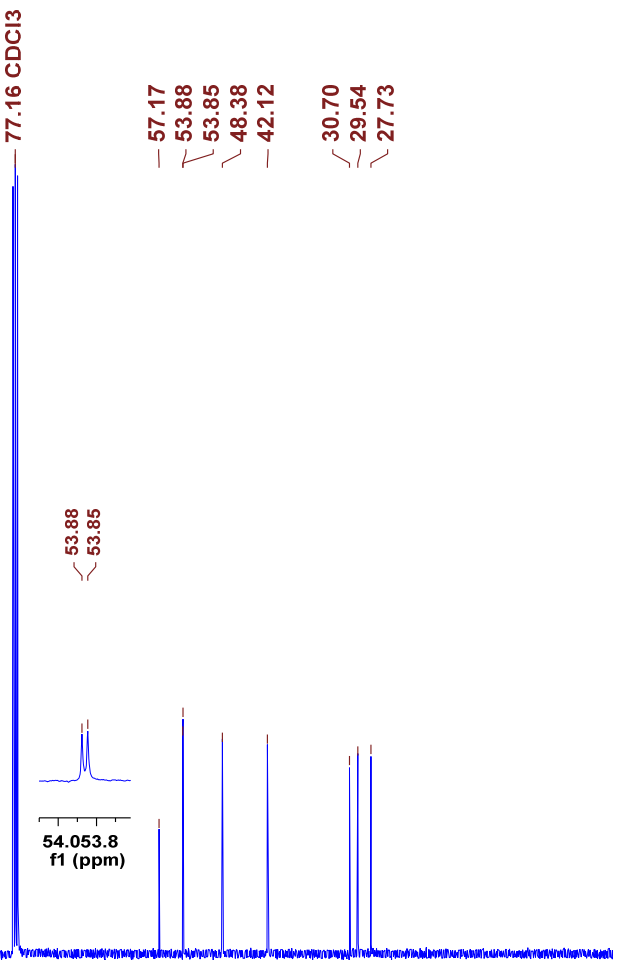




\section{References}

(S1) Goudreau, S. R.; Marcoux, D.; Charette, A. B. J. Org. Chem. 2009, 74, 470-473.

(S2) Schank, K, Lick, C. Synthesis 1983, 392-395.

(S3) Mayr, H.; Bug, T.; Gotta, M. F.; Hering, N.; Irrgang, B.; Janker, B.; Kempf, B.; Loos, R.; Ofial, A. R.; Remennikov, G.; Schimmel, H. J. Am. Chem. Soc. 2001, 123, 9500-9512.

(S4) Lin, R.; Chen, F.; Jiao, N. Org. Lett. 2012, 14, 4158-4161.

(S5) Martinez-Rich, A.; Mendoza-Diaz, G.; Joseph-Nathan, P. Appl. Spectrosc. 1996, 50, 14081412.

(S6) Yoshino, T.; Ng, F.; Danishefsky, S. J. J. Am. Chem. Soc. 2006, 128, 14185-14191.

(S7) Monte, A. P.; Marona-Lewicka, D.; Cozzi, N. V.; Nichols, D. E. J. Med. Chem. 1993, 36, 3700-3706.

(S8) Yoo, S. I.; Do, T. T.; Ha, Y. E.; Jo, M. Y.; Park, J.; Kang, Y.-C.; Kim, J. H. Bull. Korean Chem. Soc. 2014, 35, 569-574.

(S9) Tillack, A.; Rudloff, I.; Beller, M. Eur. J. Org. Chem. 2001, 523-528.

(S10) Bhojgude, S. S.; Kaicharla, T.; Biju, A. T. Org. Lett. 2013, 15, 5452-5455.

(S11) Cheng, C.; Brookhart, M. Angew. Chem., Int. Ed. 2012, 51, 9422-9424.

(S12) Chelli, S.; Troshin, K.; Lakhdar, S.; Mayr, H.; Mayer, P. Acta Crystallogr., Sect. E: Struct. Rep. Online 2016, 72, 266-268.

(S13) Altomare, A.; Burla, M. C.; Camalli, M.; Cascarano, G. L.; Giacovazzo, C.; Guagliardi, A.; Moliterni, A. G. G.; Polidori, G.; Spagna, R. J. Appl. Crystallogr. 1999, 32, 115-119.

(S14) Sheldrick, G. M. Acta Crystallogr., Sect. A: Found. Crystallogr. 2008, 64, 112-122. 